\title{
Yeast models to study mutations in SURF1 and MPV17 involved in human mitochondrial disorders
}

\author{
Dissertation
}

for the award of the degree

"Doctor rerum naturalium"

of the Georg-August-Universität Göttingen

\author{
submitted by \\ Robert Reinhold \\ from Riesa
}

Göttingen 2011 
Member of the Thesis Committee (First Reviewer):

Prof. Dr. Peter Rehling

Department of Biochemistry II

Center for Biochemistry and Molecular Cell Biology

Georg-August-Universität, Göttingen

Member of the Thesis Committee (Second Reviewer):

Prof. Dr. Mikael Simons

Cellular Neuroscience

MPI for Experimental Medicine, Göttingen

Member of the Thesis Committee:

Prof. Dr. Michael Thumm

Department of Biochemistry II

Center for Biochemistry and Molecular Cell Biology

Georg-August-Universität, Göttingen

Date of oral examination: 25.11.2011 
Herewith I declare, that I prepared this thesis on my own and with no other sources and aids than quoted.

Robert Reinhold 
Parts of this thesis have been published previously. I want to thank all co-authors and acknowledged persons for fruitful collaboration.

Reinhold, R., Bareth, B., Balleininger, M., Wissel, M., Rehling, P., and Mick, D.U. (2011). Mimicking a SURF1 allele reveals uncoupling of cytochrome $c$ oxidase assembly from translational regulation in yeast. Hum Mol Genet 20, 2379-2393. 


\section{Table of contents}

Table of contents __ v

Abbreviations _ ix

List of figures __ $x$

List of tables__

1. Introduction 1

1.1. Mitochondria

1.2. Import of nuclear-encoded proteins into mitochondria ___ 2

1.2.1. Targeting signals of mitochondrial precursor proteins ___ 3

1.2.2. Insertion of proteins into the mitochondrial inner membrane

1.3. Mitochondrial function and biogenesis _ 7

1.3.1. Mitochondria play a central role in eukaryotic cells ___ 7

1.3.2. The respiratory chain __ 7

1.3.3. The cytochrome $c$ oxidase ___ 9

1.3.4. Assembly of the cytochrome $c$ oxidase __ 10

1.4. Mitochondrial diseases ___ 13

1.4.1. Mitochondrial dysfunction leads to multisystem disorders ___ 13

1.4.2. Mutations leading to mitochondrial diseases ___ 13

1.4.3. Leigh Syndrome __ 16

1.4.4. Mitochondrial DNA Depletion Syndrome _ـ 17

1.5. Yeast as a model for mitochondrial diseases __ 18

1.5.1. Shy 1 - a model for SURF1 associated LS __ 20

1.5.2. Sym1 - a model for MPV17, a protein involved in MDDS ___ 21

1.6. Aim of this work _ 23

2. Materials and Methods ___ 24

2.1. Materials __ 24

2.1.1. Chemicals, enzymes and kit systems ___ 24

2.1.2. Microorganisms and cell lines ___ 24

2.1.3. Plasmids _ 24

2.1.4. Antibodies — 24

2.2. Cultivation of bacteria

2.3. Cultivation of yeast __ 30 
2.3.1. Growth conditions and media

2.3.2. Yeast cryo-stocks __ 31

2.3.3. Growth test of yeast strains __ 31

2.4. Culture of human cell-lines __ 31

2.5. Molecular biology methods __ 31

2.5.1. Determination of nucleic acid concentrations ___ 31

2.5.2. DNA electrophoresis _ـ 31

2.5.3. Purification of plasmids __ 32

2.5.4. PCR-amplification of DNA fragments ___ 32

2.5.5. Molecular cloning___ 32

2.5.6. Site-directed mutagenesis of plasmids ___ 32

2.5.7. Sequencing of DNA __ 33

2.5.8. Transformation of E. coli _ 33

2.5.9. Transformation of S. cerevisiae __ 33

2.6. Biochemical Methods __ 34

2.6.1. Determination of protein concentrations __ 34

2.6.2. Preparation of whole cell extracts___ 34

2.6.3. SDS-PAGE__ 34

2.6.4. BN-PAGE_— 35

2.6.5. 2D BN-PAGE/SDS-PAGE___ 35

2.6.6. Western blotting __ 35

2.6.7. Immunodetection of proteins on PVDF membranes __ 36

2.6.8. Coomassie staining___ 36

2.6.9. Colloidal Coomassie staining ___ 36

2.6.10. Drying of polyacrylamide gels ___ 36

2.6.11. Autoradiography and quantification of radiolabeled proteins __ 36

2.7. Isolation of mitochondria ___ 37

2.7.1. Isolation of yeast mitochondria __ 37

2.7.2. Isolation of mitochondria from human cells

2.8. In vitro import analysis in isolated mitochondria __ 38

2.8.1. In vitro labeling of precursor proteins __ 38

2.8.1.1. In vitro transcription __ 38

2.8.1.2. In vitro translation___ 38

2.8.1.3. Coupled in vitro transcription/translation __ 39

2.8.2. In vitro import into isolated mitochondria ___ 39

2.8.3. Stability assay after in vitro import _ـ 40 
2.10. Localization and topology of mitochondrial proteins

2.10.1. Subcellular localization analysis by fluorescence microscopy ___ 41

2.10.2. Submitochondrial localization analyses ___ 41

2.10.3. Analysis of membrane association by carbonate treatment __ 41

2.11. Purification of mitochondrial protein complexes___ 42

2.11.1. IgG-chromatography _ـ 42

2.11.2. Co-immunoprecipitation___ 42

2.12. Electrophysiological characterization of mitochondrial proteins _ 42

2.12.1. Isolation of proteins from yeast mitochondria for electrophysiological characterization _ـ 42

2.12.2. Electrophysiology___ 43

3. Results _ 44

3.1. SHY1 as a model for SURF1 mutations, identified in Leigh Syndrome patients __ 44

3.1.1. SHY1 mutations, mimicking patient SURF1 alleles lead to cytochrome $c$ oxidase deficiency __ 44

3.1.1.1. Yeast models for SURF1 mutations show respiratory deficiency ___ 44

3.1.1.2. SHY1 mutant strains lack cytochrome $c$ oxidase___ 45

3.1.1.3. Mutations affecting $\mathrm{G}^{137}$ of Shy1 lead to reduced abundance of Shy $1 \_47$

3.1.2. Import and stability of Shy1 in yeast mitochondria ___ 48

3.1.2.1. Mutant Shy1 is efficiently imported into yeast mitochondria___ 48

3.1.2.2. Mutation of $\mathrm{G}^{137}$ in Shy1 leads to rapid turnover after import ___ 50

3.1.2.3. Shy $1^{\mathrm{Y} 344 \mathrm{D}}$ maintains the physiologically correct topology $\_51$

3.1.3. Import and stability of SURF1 in human mitochondria _ 52

3.1.3.1. Establishment of an in vitro import assay of SURF1 _ 52

3.1.3.2. Mutant SURF1 is efficiently imported into mitochondria

3.1.3.3. Mutations affecting $\mathrm{G}^{124}$ in SURF1 lead to degradation of the mature protein ___ 54

3.1.3.4. SURF $1{ }^{\mathrm{Y} 274 \mathrm{D}}$ association with cytochrome $c$ oxidase assembly intermediates is affected _ 55

3.1.4. Shy $1^{\mathrm{Y} 344 \mathrm{D}}$ uncouples Cox 1 expression from cytochrome $c$ oxidase assembly ___ 58

3.1.4.1. Shy $1^{\mathrm{Y} 344 \mathrm{D}}$ permits Cox1 expression

3.1.4.2. Shy $1^{\mathrm{Y} 344 \mathrm{D}}$ releases Cox1 from translational feedback regulation $\_59$

3.1.4.3. Cox 1 is rapidly degraded in shy $1^{Y 344 D}$ cells 60

3.1.4.4. Shy $1^{\mathrm{Y} 344 \mathrm{D}}$ has a dominant negative effect on Cox1 stability ___ 62

3.1.5. Mitochondrial quality control by mitophagy ___ 63

3.2. Sym1, a model for MPV17-associated Mitochondrial DNA Depletion Syndrome _ 65

3.2.1. Sym1 is a polytopic protein of the mitochondrial inner membrane ___ 65

3.2.1.1. MPV17 patient mutations affect highly conserved amino acids ___ 65

3.2.1.2. SYMI deletion does not affect respiratory competence in yeast 
3.2.1.3. Localization and topology of Sym1

3.2.2. Formation and assembly of Sym1 complexes

3.2.2.1. Sym1 forms high molecular membrane complexes __ 69

3.2.2.2. Establishment of an in vitro import assay of Sym1 70

3.2.2.3. Sym1 assembly is not dependent on the TIM22-translocase __ 72

3.2.2.4. Sym1 assembly is dependent on functional TIM23-translocase ___ 72

3.2.2.5. The influence of small Tim proteins on Sym1 assembly _ _ 75

3.2.2.6. Mapping Sym1 domains, necessary for its import and assembly ___ 76

3.2.3. Modeling MPV17 patient mutations using SYM1 ___ 77

3.2.3.1. Mutant Sym1 proteins are stable in mitochondria __ 77

3.2.3.2. Sym $1^{\mathrm{R} 51 \mathrm{Q}}$ and $\mathrm{Sym} 1^{\mathrm{N} 172 \mathrm{~K}}$ assembles into high molecular complexes ___ 78

3.2.3.3. Assembly of mutant Sym1 proteins __ 79

3.2.4. Sym1 forms a pore in the mitochondrial inner membrane ___ 80

3.2.4.1. Purification of Sym1 from yeast mitochondria ___ 80

3.2.4.2. Electrophysiological characterization of Sym1 81

4. Discussion _ 84

4.1. Molecular pathology of SURF1 point mutations __ 84

4.2. SURF1 ${ }^{\mathrm{Y} 27 \mathrm{D}}$ reveals novel cytochrome $c$ oxidase assembly intermediates ___ 87

4.3. Association of SURF1 with mature cytochrome $c$ oxidase

4.4. Shy $1^{\mathrm{Y344D}}$ uncovers dual functionality of Shy1

4.5. Degradation of assembly intermediates of the cytochrome $c$ oxidase

4.6. Sym1 is a mitochondrial member of a conserved protein family __ 91

4.7. A controversy about the $\operatorname{sym} 1 \Delta$ phenotype

4.8. Sym1 complexes in the mitochondrial inner membrane __ 93

4.9. Sym1 as a model for the assembly of polytopic inner membrane proteins __ 94

4.10. SYM1 models for patient MPV17 point mutations _ 95

4.11. The channel activity of Sym1 and its implications for MDDS

4.12. Conclusion __

5. Summary__ 99

Bibliography_ 100

Acknowledgements__ 115

Curriculum Vitae _ 116 


\section{Abbreviations}

$\Delta \psi \quad$ membrane potential

ATP adenosine triphosphate

CoQ Coenzyme $\mathrm{Q}_{10}$, ubiquinone

BN-PAGE Blue-Native polyacrylamide gelelectrophoresis

BSA bovine serum albumin

DNA deoxyribonucleic acid

EDTA ethylenediaminetetraacetic acid

FAD flavin adenine dinucleotide

HEPES 4-(2-hydroxyethyl)-1-piperazineethanesulfonic acid

HRP horseradish peroxidase

IgG Immunoglobulin $\mathrm{G}$

IMS inter membrane space

LS Leigh Syndrome

MDDS Mitochondrial DNA Depletion Syndrome

MIM mitochondrial inner membrane

MOM mitochondrial outer membrane

MOPS 3-(N-morpholino)propanesulfonic acid

mRNA messenger ribonucleic acid

NADH nicotinamide adenine dinucleotide

PCR polymerase chain reaction

PMSF phenylmethylsulfonyl fluoride

PVDF polyvinylidene fluoride

SDS-PAGE sodium dodecyl sulfate polyacrylamide gelelectrophoresis

TIM translocase of the inner membrane

TOM translocase of the outer membrane

Tris tris(hydroxymethyl)-aminomethan 


\section{List of figures}

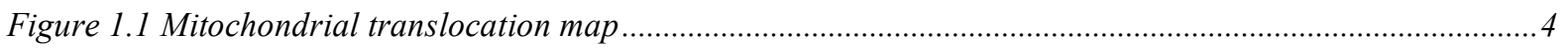

Figure 1.2 Components of the inner membrane insertion machineries ...........................................................5

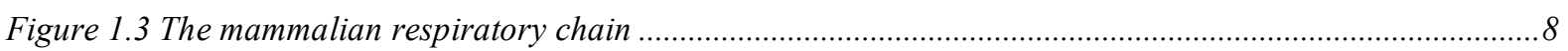

Figure 1.4 Translational feed back regulation of Coxl synthesis in yeast .................................................12

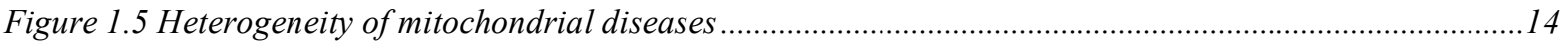

Figure 3.1 Mimicking highly conserved SURF1 patient mutations in yeast leads to respiratory deficiency........45

Figure 3.2 Point mutations in SHY1 lead to lack of cytochrome c oxidase .......................................................46

Figure 3.3 SHY1 point mutations lead to reduced abundance of Shyl and core cytochrome c oxidase subunits 47

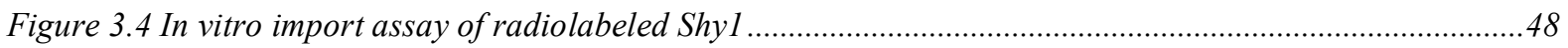

Figure 3.5 Mitochondrial import of mutant Shyl proteins is not affected .......................................................49

Figure 3.6 Shy $1^{\text {GI37E }}$ and Shy $1^{\text {Gl37R }}$ are destabilized in yeast mitochondria ......................................................50

Figure 3.7 Shy $1^{Y 344 D}$ is correctly inserted in the mitochondrial inner membrane ..............................................51

Figure 3.8 In vitro import of SURF1 into human mitochondria ..................................................................52

Figure 3.9 Mutant SURF1 imports efficiently into human mitochondria ..........................................................53

Figure 3.10 Exchange of $G^{124}$ in SURF1 leads to rapid degradation in mitochondria...........................................55

Figure 3.11 Altered association of SURF $1^{Y 274 D}$ to cytochrome c oxidase assembly intermediates.......................57

Figure 3.12 shyl $^{Y 344 D}$ cells efficiently translate mitochondria-encoded proteins...................................................59

Figure 3.13 Analysis of early cytochrome c oxidase assembly intermediates in yeast .....................................60

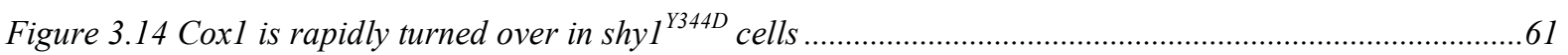

Figure 3.15 Shy $1^{Y 344 D}$ has a dominant negative effect on Cox 1 stability in yeast ...............................................62

Figure 3.16 Analysis of Uth1, the suggested mitophagy receptor in the outer membrane ...................................64

Figure 3.17 Patient mutations in MPV17 affect conserved residues in putative transmembrane spans ...............66

Figure 3.18 Cells lacking Sym1 show respiratory competence ….................................................................67

Figure 3.19 Sym 1 is a protein of the mitochondrial inner membrane ..................................................................69

Figure 3.20 High molecular weight membrane complexes of Sym 1 ..............................................................70

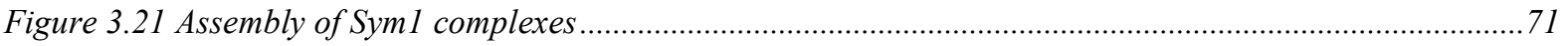

Figure 3.22 Sym1 assembly in TIM22 mutant mitochondria .............................................................................73

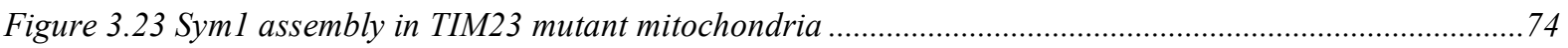

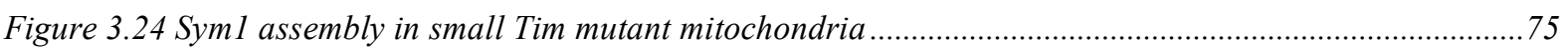

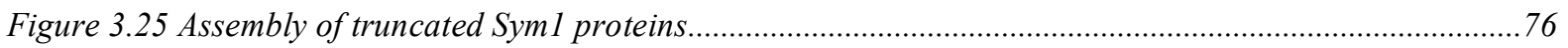

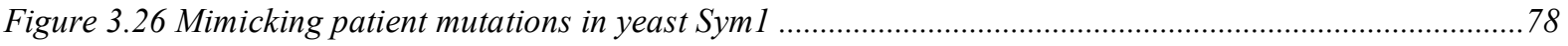

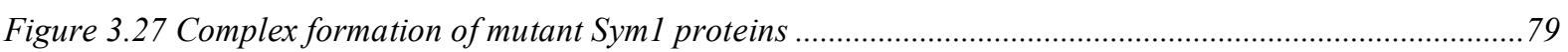

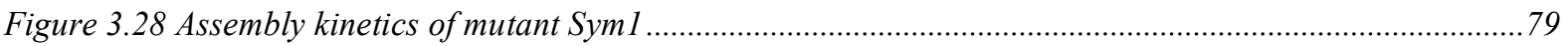

Figure 3.29 Purification of Sym1 for electrophysiological characterization ....................................................80

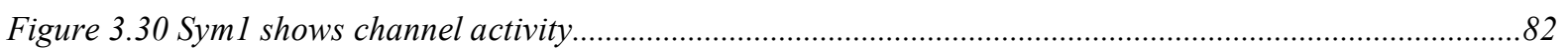

Figure 3.31 Cation selectivity and specificity of the Sym 1-pore ...................................................................8 


\section{List of tables}

Table 1.1 Pathogenic missense mutations affecting SURF1 and MPV17 .........................................................19

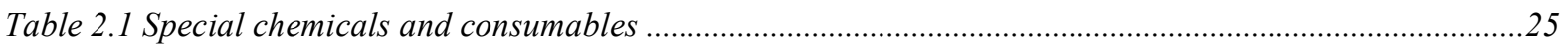

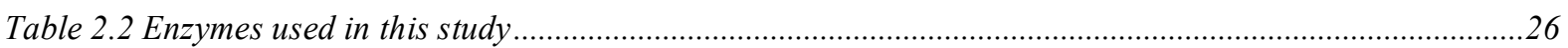

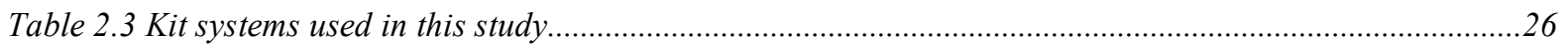

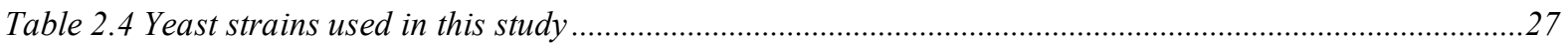

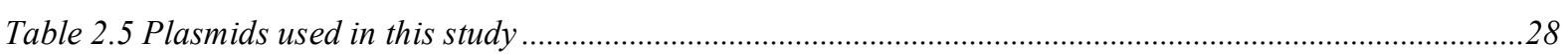

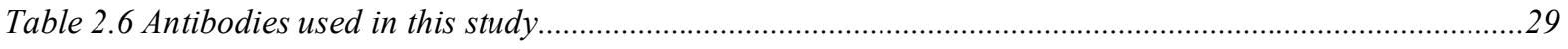

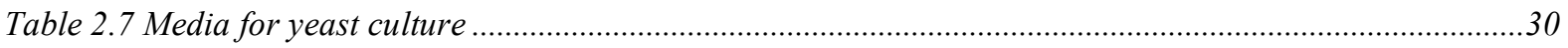

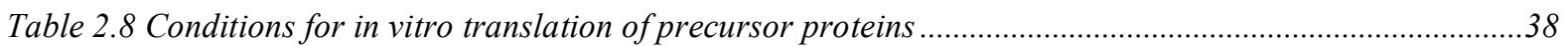




\section{Introduction}

\subsection{Mitochondria}

Eukaryotic cells are characterized by a complex compartmentalization. The compartments or organelles are separated by lipid membranes. These membranes form a diffusion barrier that is necessary for the integrity of the organelles. Within the different organelles optimal enzyme composition as well as $\mathrm{pH}$ and redox conditions can be maintained without interfering with the remainder of the cell. This provides multiple specialized environments for the multitude of cellular activities. In addition the biological membranes are required for concentration gradients of solutes or metabolites. These concentration gradients can be used for storage of metabolites as well as signaling processes as exemplified in calcium signaling (Clapham, 2007). Moreover, biological membranes are the site of coupling biochemical reactions to the generation or consumption of gradients and thereby converting chemical energy into potential energy or vice versa (e.g. the respiratory chain). Organelles also protect their content or environment from damage. Examples for this principle are the nucleus, protecting the genome from mutagens or the lysosomes that contain proteases, amylases and other degenerative enzymes (de Duve, 2005; Luzio et al., 2007; Mekhail and Moazed, 2010). In the middle of the $19^{\text {th }}$ century cytologists first identified the diversity of subcellular structures. By the end of the $19^{\text {th }}$ century the uniformity of eukaryotic compartmentalization was recognized by the discovery of ubiquitous structures. Since then the identification and characterization of the nucleus, mitochondria, endoplasmic reticulum and other organelles has given rise to our current understanding of eukaryotic cell biology (Palade, 1964).

Mitochondria, present in virtually all eukaryotes are essential organelles involved in energy metabolism and other basic cellular processes (see section 1.3.1). The mitochondrial subcompartments are characterized by two mitochondrial membranes. The matrix is enclosed by the mitochondrial inner membrane and the inter membrane space separates the inner and outer membrane. The mitochondrial inner membrane forms cristae, characteristic invaginations that increase the membrane surface. Within a cell, mitochondria form tubular networks that are highly dynamic. Interestingly mitochondria can divide, fuse and even migrate along cytoskeletal structures. Mitochondrial morphology and dynamics are a focus of current mitochondrial research (Liesa et al., 2009; Okamoto and Shaw, 2005). 
The origin of mitochondria is believed to be an endosymbiotic event of a respiring prokaryote. The nature of the host cell as well as the early evolution of mitochondria is currently under controversial debate (Gray et al., 1999; Lithgow and Schneider, 2010; Szklarczyk and Huynen, 2010). The endosymbiont however, was characterized by comparative genomics to resemble $\alpha$-proteobacteria (Andersson et al., 1998).

During mitochondrial evolution most endosymbiotic genes were transferred to the host genome. Accordingly, these genes encode for proteins that are synthesized in the cytosol and need to be imported into mitochondria (see section 1.2). However, few genes are still mitochondria-encoded (e.g. 13 in humans; 8 in Sacharomyces cerevisiae). In most species the mitochondrial genome is a circular DNA molecule, containing very compact genetic information. Furthermore the mitochondrial DNA is an extrachromosomal element that is inherited in a non mendelian, mostly maternal fashion. Although the genes encoded by the mitochondrial genome vary between species, for not yet fully understood reasons, there is a minimal set of proteins that is mitochondria-encoded in all eukaryotes (Wallace, 2007). Interestingly, for the expression of the small number of mitochondria-encoded proteins, a full set of DNA replication and expression machineries is maintained in mitochondria. Moreover the dual genetic origin of mitochondrial proteins requires a coordination of the mitochondrial protein expression and import during organellar biogenesis.

\subsection{Import of nuclear-encoded proteins into mitochondria}

The mitochondrial proteome comprises about 1000 proteins (Sickmann et al., 2003; Taylor et al., 2003). However, the organellar genome encodes only for about $1 \%$ of these polypeptides. Hence, the vast majority of mitochondrial proteins is synthesized on cytosolic ribosomes and subsequently imported into mitochondria. In contrast to protein import into the endoplasmic reticulum, mitochondrial protein import occurs posttranslationally. Furthermore, unlike nuclear or peroxisomal import, proteins remain in an unfolded state prior to translocation.

Protein import into one of the four mitochondrial subcompartments is a highly coordinated and complex mechanism that involves multiple translocase machineries (Fig 1.1) (Becker et al., 2009; Chacinska et al., 2009; van der Laan et al., 2010).

In brief, mitochondrial precursor proteins are synthesized on cytosolic ribosomes, bound by cytosolic chaperones and guided to the translocase of the outer membrane (TOM). The TOM complex is the general import pore for virtually all mitochondrial proteins. It contains 
receptor proteins (Tom20, Tom22, Tom70) and Tom40 that forms a $\beta$-barrel channel for protein translocation (Ahting et al., 2001; Becker et al., 2005; Model et al., 2008). After the precursor is translocated across the outer membrane it is subsequently transported via specialized import machineries dependent on the targeting signal and the submitochondrial fate of the precursor. Noteworthy, two different translocases of the inner membrane (TIM) have evolved, the presequence translocase (TIM23) and the carrier translocase (TIM22). Both TIM translocases are multimeric protein complexes that are capable to insert precursor proteins into the mitochondrial inner membrane (see section 1.2.2). In addition, TIM23 also facilitates translocation into the matrix and can release proteins into the inter membrane space. The sorting and assembly machinery (SAM) accomplishes insertion of $\beta$-barrel proteins into the mitochondrial outer membrane and the mitochondrial inter membrane space assembly machinery (MIA) is required for maturation of cysteine-containing small inter membrane space proteins.

\subsubsection{Targeting signals of mitochondrial precursor proteins}

Cytosolic precursors of mitochondrial proteins contain specific signals that target these proteins for import to the particular submitochondrial localization (Fig 1.1).

The most common mitochondrial targeting signals are cleavable presequences that are present in about $70 \%$ of mitochondrial precursors (Vögtle et al., 2009). The presequence is located at the $\mathrm{N}$-terminus of the precursors and forms an amphipathic $\alpha$-helix. Presequences target proteins via TIM23 into the matrix. However, some proteins contain presequences that are followed by a hydrophobic sorting signal. This sorting signal arrests translocation and proteins are laterally released into the inner membrane (Glick et al., 1992). The inner membrane protease cleaves off the hydrophobic sorting signal of some of these proteins, which subsequently remain soluble in the inter membrane space. In most cases the presequence can be cleaved by the matrix processing peptidase, resulting in an $\mathrm{N}$-terminal truncation of the mature protein.

An exception are presequence-like internal targeting signals, identified in a small number of proteins such as Bcs1, Mdj2 and Pam18 (Fölsch et al., 1996; Mokranjac et al., 2003; Truscott et al., 2003; Westermann and Neupert, 1997). In this case a hydrophobic sequence is followed by a positively charged presequence-like helix. These two domains form a hairpin 


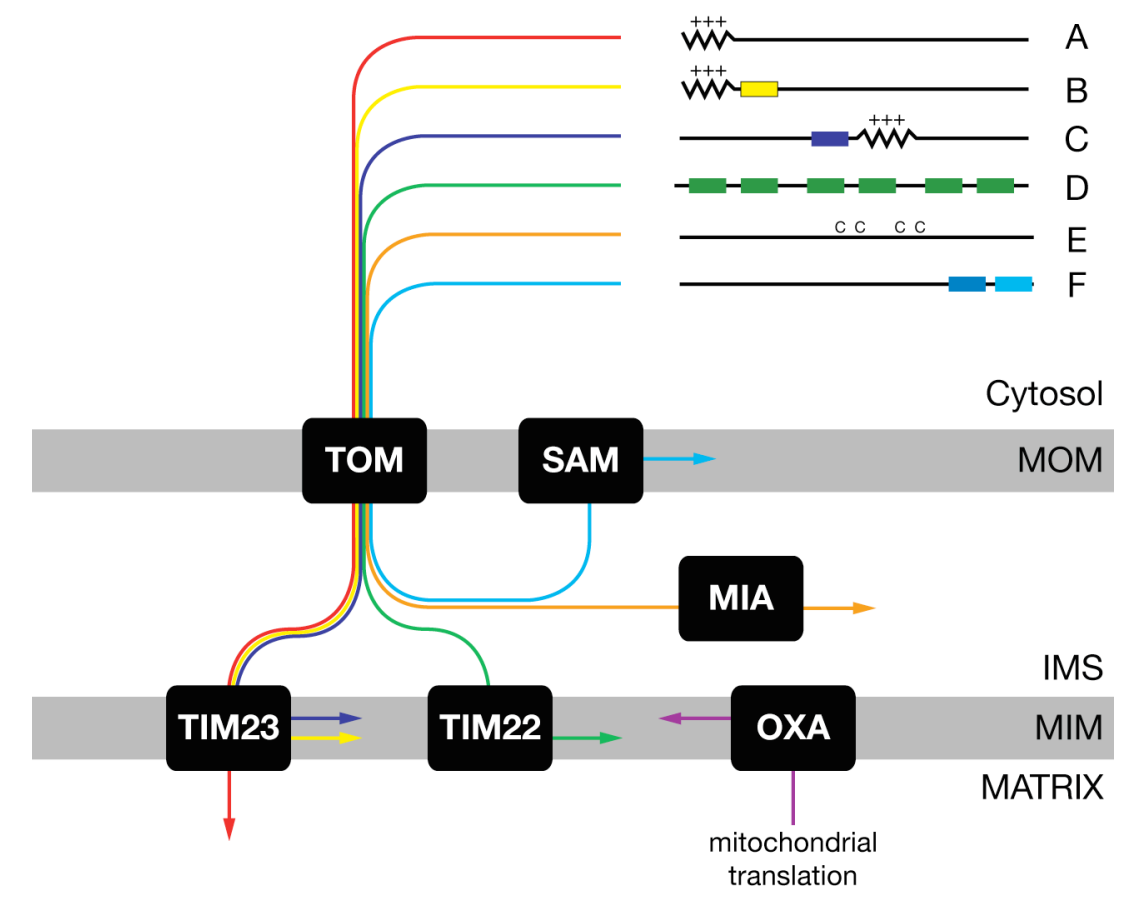

Figure 1.1 Mitochondrial translocation map

Cytosolic precursor proteins are transported via specialized import machineries into mitochondria. The translocase of the outer membrane (TOM) facilitates transport across the outer membrane. Subsequently the translocases of the inner membrane (TIM23, TIM22), the sorting and assembly machinery (SAM) or the mitochondrial inter membrane space assembly machinery (MIA) are required for transport to the respective submitochondrial destination and maturation of the protein. Various mitochondrial targeting signals target proteins for mitochondrial import: A classical presequence (A) mediates transport into the matrix. A presequence followed by a sorting signal (B), a presequence-like internal motive (C) or multiple internal targeting signals (D) can target proteins into the inner membrane. Cysteine containing internal signals (E) are recognized by MIA and the C-terminal $\beta$-signal targets proteins to SAM. In addition, proteins can be transported by the mitochondrial export machinery (OXA). MOM, mitochondrial outer membrane; IMS, inter membrane space; MIM, mitochondrial inner membrane.

loop that mimics an amphipathic presequence and targets the protein to TIM23 mediated insertion into the inner membrane.

Members of the family of inner membrane metabolite carriers, such as the ADP/ATP carrier (AAC) and the phosphate carrier have six transmembrane spans and their targeting signals are distributed across the entire length of the protein (Wiedemann et al., 2001). These proteins are transported via TIM22 (Pfanner and Neupert, 1987; Rehling et al., 2004).

Furthermore, many small proteins of the mitochondrial inter membrane space contain cysteine rich $\mathrm{Cx}_{3} \mathrm{C}$ or $\mathrm{Cx}_{9} \mathrm{C}$ motifs for targeting to the MIA pathway (Herrmann and Köhl, 2007; Stojanovski et al., 2008). Moreover, precursors of ß-barrel outer membrane proteins contain a C-terminal targeting sequence that targets these proteins to SAM (Becker et al., 2008). 


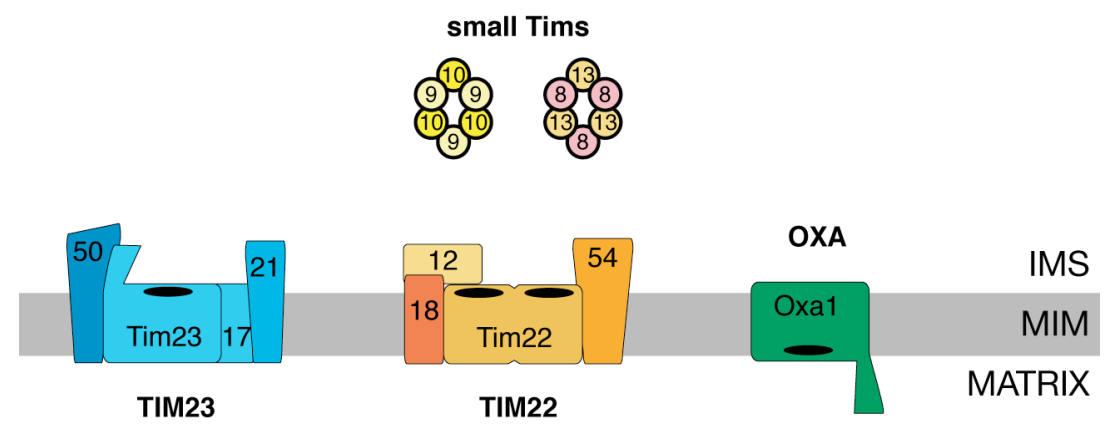

Figure 1.2 Components of the inner membrane insertion machineries

Mitochondrial inner membrane proteins are inserted into the membrane by TIM23, TIM22 or the OXA machinery. The composition of these multimeric complexes is indicated. Additionally, small Tims function as chaperones and prevent aggregation in the inter membrane space.

Presequences are well characterized and can be predicted based on the primary sequence. Alternative mitochondrial targeting sequences are a focus of current research.

\subsubsection{Insertion of proteins into the mitochondrial inner membrane}

For this thesis the insertion of proteins into the mitochondrial inner membrane is of special interest (Fig 1.2). As previously mentioned, nuclear-encoded proteins can be inserted into the mitochondrial inner membrane via both TIM translocases, TIM23 and TIM22. Furthermore mitochondria-encoded proteins are inserted into the inner membrane via the mitochondrial export machinery (OXA), which is also involved in the so-called conservative sorting pathway.

Insertion of presequence-containing precursors with a sorting signal is mediated by TIM23. The core complex of TIM23 consists of the protein conducting channel, formed by Tim23 and Tim17, and Tim50 that regulates channel opening and passes the precursor from TOM to the TIM23 translocase (Geissler et al., 2002; Meinecke et al., 2006; Truscott et al., 2001; Yamamoto et al., 2002). Additionally, Tim21 is involved in the interaction of TOM with TIM23 and promotes presequence transmission (Chacinska et al., 2005; Mokranjac et al., 2005). The positively charged presequence is transported through TIM23 by an electrophoretic force, generated by the membrane potential $(\Delta \psi)$. Subsequently the translocation is arrested by the hydrophobic sorting signal and the precursor is released laterally into the inner membrane (Glick et al., 1992). Although lateral release in the inner membrane is not yet fully understood it was shown that this translocation process requires the 
membrane potential $(\Delta \psi)$, Tim21 and Tim17 (Chacinska et al., 2005; van der Laan et al., 2007).

The TIM22 translocase transports members of the metabolite carrier family. These hydrophobic proteins are shuttled to TIM22 by so called small Tims, hexameric inter membrane space chaperones. The Tim9-Tim10 complex is the major inter membrane space chaperone and guides not only metabolite carriers and other inner membrane proteins to TIM22 but also ß-barrel proteins to SAM (Curran et al., 2002a; Webb et al., 2006; Wiedemann et al., 2006a). In addition, the non-essential Tim8-Tim13 complex also forms a hexameric inter membrane space chaperone and guides substrates such as Tim23 to the TIM22 translocase (Curran et al., 2002b; Davis et al., 2007). The chaperone bound precursor is recruited to TIM22 by Tim12, a peripheral subunit of TIM22 (Sirrenberg et al., 1998). The membrane integral part of TIM22 consists of Tim18, Tim54 and Tim22, which forms the channel for protein translocation (Rehling et al., 2003). Dependent on the membrane potential $(\Delta \psi)$ the precursor is inserted into the TIM22 translocase, laterally released into the membrane in an unresolved manner and then assembles into functional complexes.

Another machinery for protein insertion into the inner membrane is the mitochondrial export machinery (OXA - for oxidase assembly). This complex consists of Oxa1 as core subunit (Kohler et al., 2009; Nargang et al., 2002). Oxal is a homolog of the bacterial YidC, which facilitates protein insertion into the bacterial membrane on its own and also cooperates with the bacterial Sec-translocase (du Plessis et al., 2011; Wang and Dalbey, 2011). Oxa1 mediates the conservative sorting pathway of presequence containing inner membrane proteins that first are transported into the matrix and subsequently are inserted into the inner membrane dependent on the membrane potential (Hell et al., 1998; Rojo et al., 1995). Additionally, Oxal is also involved in membrane insertion of multispanning proteins with presequences and hydrophobic sorting signals (Bohnert et al., 2010). Mitochondria-encoded proteins in higher eukaryotes are almost exclusively hydrophobic proteins. Oxal mediates the insertion of these proteins in a cotranslational manner (Hell et al., 2001; Ott and Herrmann, 2010). The mitochondrial ribosome binds to the C-terminal extension of Oxa1. Subsequently the nascent chain is transferred to Oxal and the protein is inserted into the membrane during synthesis. For this process additional factors, such as Mba1, Cox18 and translational activators are required (Mick et al., 2011; Towpik, 2005). 


\subsection{Mitochondrial function and biogenesis}

\subsubsection{Mitochondria play a central role in eukaryotic cells}

Mitochondria are well known for their role in the energy metabolism of eukaryotic cells. Primarily, mitochondria contribute to cellular energy homeostasis by production of ATP via the oxidative phosphorylation system, which is energized by the electron flux of the respiratory chain.

In addition to the role in bioenergetics, the importance of mitochondria in various other biochemical processes became apparent. Examples are the $\beta$-oxidation of fatty acids and the urea cycle, which at least partially take place in mitochondria. In addition, mitochondria are essential for the biogenesis of iron-sulfur clusters as well as heme groups (Ajioka et al., 2006; Lill, 2009). Interestingly, due to their involvement in iron-sulfur cluster biogenesis mitochondria or organelles derived from degenerated mitochondria (mitosomes, hydrogenosomes) are also indispensable in organisms that do not depend on oxidative energy generation such as Trichomonas vaginalis and Giardia lamblia, unicellular eukaryotic parasites (Shiflett and Johnson, 2010; van der Giezen and Tovar, 2005).

In mammals, mitochondria also play a role in heat generation by uncoupling of the respiratory chain from ATP production (Rousset et al., 2004). Furthermore the involvement of mitochondria in apoptosis, ageing and cancer indicates the essential role of mitochondria in regulation of cell growth and death (Balaban et al., 2005; Gogvadze et al., 2008; Guarente, 2008; Seo et al., 2010).

The multitude of mitochondrial functions explains their essential role for eukaryotic cells and the pleiotropic effects of mitochondrial dysfunctions (see section 1.4).

\subsubsection{The respiratory chain}

In higher eukaryotes, the main source of energy is the oxidative phosphorylation system. Oxidative phosphorylation depends on the respiratory chain in the mitochondrial inner membrane that transfers electrons from reducing equivalents (NADH, succinate) to molecular oxygen (Fig 1.3). The energy of this electron flux is used to generate a proton gradient across the mitochondrial inner membrane (membrane potential, $\Delta \psi$ ). This proton gradient subsequently drives ATP synthesis via the $\mathrm{F}_{1} \mathrm{~F}_{\mathrm{O}}$-ATPase. The respiratory chain consists of four multi subunit complexes. Although functionally and structurally conserved, the 


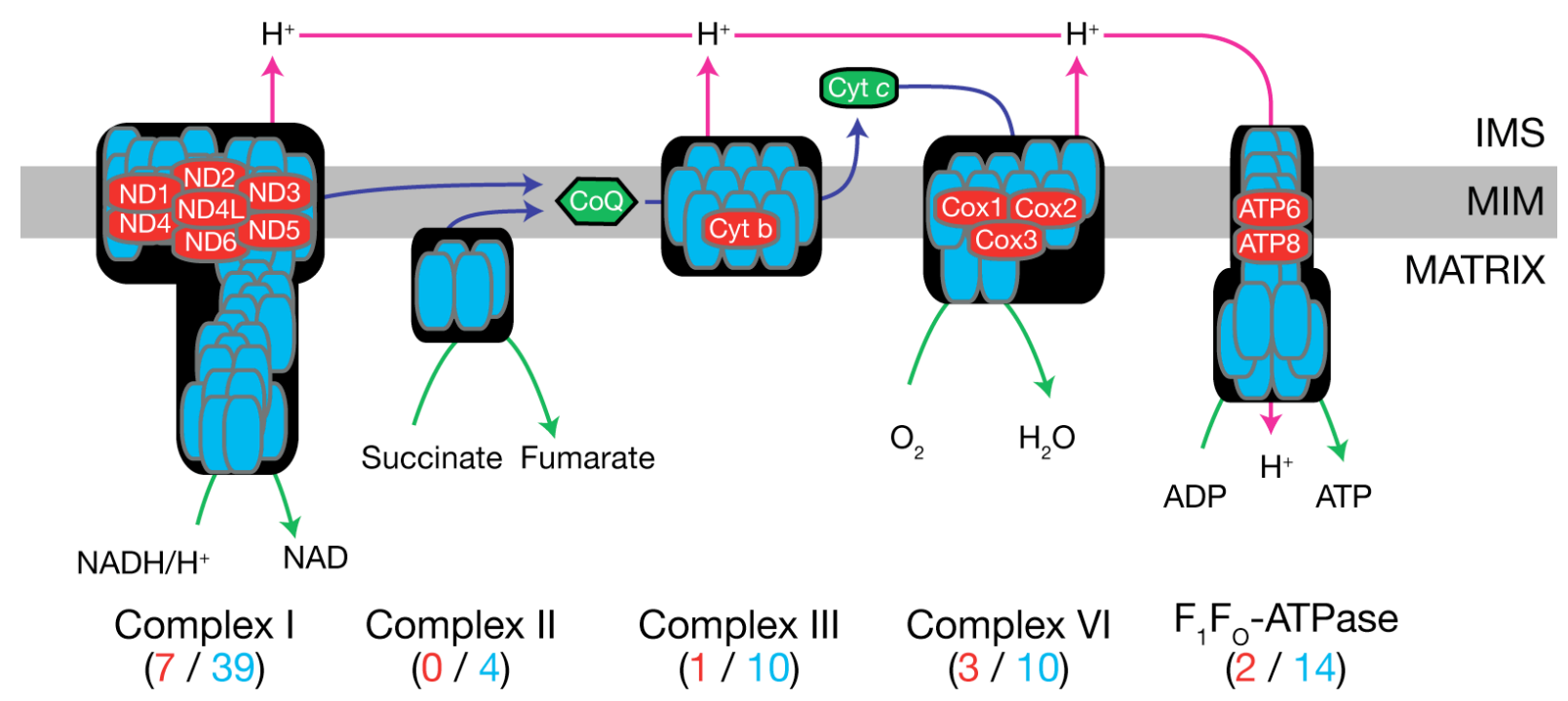

Figure 1.3 The mammalian respiratory chain

The respiratory chain is formed by multimeric complexes in the mitochondrial inner membrane. These complexes contain mitochondrial (depicted in red) and nuclear (blue) encoded subunits. The electron transport chain from NADH or succinate to oxygen via complex I to IV energizes the generation of a proton gradient, which drives ATP synthesis by the $\mathrm{F}_{1} \mathrm{~F}_{\mathrm{O}}$-ATPase.

composition of these complexes varies between species. Therefore, in the following, the mammalian respiratory chain is used as an example if not stated otherwise. All of the respiratory chain complexes are integral membrane complexes and, except for complex II, contain mitochondria-encoded core subunits and additional nuclear-encoded subunits. Electrons are transferred between the complexes by electron shuttles in the inner membrane (ubiquinone, also termed $\mathrm{CoQ}$ ) and inter membrane space (cytochrome $c$ ).

The NADH dehydrogenase (complex I), which is not present in S. cerevisiae, transfers two electrons from NADH to ubiquinone. The energy of this process is used for proton translocation from the matrix to the inter membrane space, giving rise to the proton gradient. Complex I is the biggest respiratory chain complex and consists of 45 or 46 subunits, ironsulfur clusters as well as flavin mononucleotide as redox centers (Carroll et al., 2006). Interestingly only 14 subunits form the functional core of the complex, which is conserved from prokaryotes to man (Brandt, 2006). Seven of these core subunits are highly hydrophobic and are encoded by the mitochondrial genome, whereas the other 7 subunits are hydrophilic and encoded in the nucleus. Interestingly in plants and many fungi alternative NADH dehydrogenases have evolved (Kerscher, 2000). These enzymes directly feed electrons into the respiratory chain without proton pumping. Examples are Ndi1, Nde1 and Nde2, the alternative NADH dehydrogenases of S. cerevisiae (Luttik et al., 1998; Marres et al., 1991). 
The succinate dehydrogenase (complex II) transfers electrons from succinate or fatty acids via FAD to ubiquinone. Complex II, the smallest respiratory chain complex, does not participate in proton pumping and only consists of four nuclear-encoded subunits (Hagerhall, 1997; Sun et al., 2005).

The lipophilic electron carrier ubiquinone is oxidized by the cytochrome $b c_{1}$ complex (complex III), which transfers the electrons to cytochrome $c$ and pumps protons into the inter membrane space. Complex III consists of 11 subunits, one of which (cytochrome $b$ ) is encoded by the mitochondrial genome. In addition it contains heme $b$, heme $c_{l}$ and iron sulfur clusters as redox centers (Iwata et al., 1998; Xia et al., 1997).

Reduced cytochrome $c$ is subsequently passed to the cytochrome $c$ oxidase (complex IV), which transfers the electrons to molecular oxygen. Thereby, the cytochrome $c$ oxidase generates water and pumps protons into the inter membrane space (see next section).

The proton gradient, generated by the respiratory chain drives not only protein import and other transport processes but is also used by the $\mathrm{F}_{1} \mathrm{~F}_{\mathrm{O}}$-ATPase. This complex uses the electrochemical gradient to catalyze ATP synthesis (Nakamoto et al., 2008; Stock et al., 2000).

Interestingly, the above-mentioned complexes form higher oligomers, so called supercomplexes or respirasomes (Acin-Perez et al., 2008; Schägger and Pfeiffer, 2000). For example, in yeast a dimer of complex III associates to one ore two copies of complex IV $\left(\mathrm{III}_{2} \mathrm{IV}, \mathrm{III}_{2} \mathrm{IV}_{2}\right)$ and the ATPase is present in a dimeric form $\left(\mathrm{V}_{2}\right)$ (Arnold et al., 1998). In mammals a dimer of complex III can associate to complex I and complex IV to form various higher oligomers (Schägger and Pfeiffer, 2000). The function of supercomplex formation is not yet fully understood. However, it has been suggested that supercomplex formation might improve electron transfer between the complexes and that they are involved in mitochondrial ultrastructure (Acin-Perez et al., 2008; Wittig and Schägger, 2009; Zick et al., 2009).

\subsubsection{The cytochrome $c$ oxidase}

The cytochrome $c$ oxidase, the terminal enzyme complex of the respiratory chain, couples the electron transport from cytochrome $c$ to molecular oxygen to the translocation of protons from the matrix into the inter membrane space. The cytochrome $c$ oxidase consists of 11 subunits in $S$. cerevisiae or 13 subunits in human and belongs to the family of heme-copper oxidases (Ferguson-Miller and Babcock, 1996). Interestingly, the crystal structure of the 
dimeric bovine cytochrome $c$ oxidase (Tsukihara et al., 1996) revealed a very compact structure of the 13 subunits with cofactors buried deeply inside the complex.

The three core subunits, Cox1, Cox 2 and Cox 3 are mitochondria-encoded in most eukaryotes and highly conserved in all respiring organisms (Castresana et al., 1994). These core subunits contain heme and copper cofactors as redox centers. Interestingly, most respiring prokaryotes only have these three core subunits, which are sufficient for cytochrome $c$ oxidase activity, indicating their functional importance. Hence, the structure and function of the core subunits has been analyzed using prokaryotic cytochrome $c$ oxidase as a model (Iwata et al., 1995; Svensson-Ek et al., 2002).

Cox 1 is the central subunit of the cytochrome $c$ oxidase and contains 12 transmembrane helices and heme $a$, heme $a_{3}$ as well as the $\mathrm{Cu}_{\mathrm{B}}$ site as redox centers. Furthermore Cox 1 is involved in proton pumping and contains two proton translocating pores (D-channel, Kchannel) (Gennis, 1998). Moreover, Cox1 is also the central subunit for cytochrome $c$ oxidase assembly (see next section) and absence of Cox1 leads to rapid degradation of the other core cytochrome $c$ oxidase subunits.

Cox2, a protein with two membrane spans and an inter membrane space domain, coordinates the $\mathrm{Cu}_{\mathrm{A}}$ site as redox active center. The $\mathrm{Cu}_{\mathrm{A}}$ site together with heme $a$ forms the entry site for electrons in the cytochrome $c$ oxidase. Little is known about Cox3, which has seven transmembrane spans and does not participate in proton pumping. However, studies on bacterial Cox 3 homologs indicated that Cox 3 is involved in proton uptake at physiological $\mathrm{pH}$ values (Gilderson et al., 2003).

The nuclear-encoded subunits show less sequence conservation and are believed to be required for complex assembly and stabilization. In addition there are isoforms of nuclearencoded subunits, which are expressed tissue specifically (e.g. COX6A1/COX6A2 in mammals) or depended on environmental conditions (e.g. Cox $5 \mathrm{a} / \mathrm{Cox} 5 \mathrm{~b}$ in yeast), which probably modulate cytochrome $c$ oxidase activity (Fabrizi et al., 1992; Hodge et al., 1989).

\subsubsection{Assembly of the cytochrome $c$ oxidase}

The assembly of the cytochrome $c$ oxidase involves the coordination of multiple subunits of dual genetic origin and the insertion of heme and copper cofactors. This process occurs in an assembly line like manner and requires more than 20 assembly factors (Herrmann and Funes, 2005; Khalimonchuk and Rödel, 2005; Mick et al., 2011). The assembly factors are present 
in substoichiometric amounts and function at different steps of the assembly process. Some assembly factors regulate the expression of mitochondria-encoded subunits, others are required for cofactor insertion or have a chaperone function.

The assembly process starts with the expression of the mitochondria-encoded core subunit Cox1. In yeast, expression of mitochondria-encoded subunits is tightly controlled by translational activators that bind the 5' untranslated regions of the mRNA (Fig 1.4). In mammals, there are no extensive 5' untranslated regions in mitochondrial mRNAs and translational control is less understood. Although possible mammalian COX1 translation factors, such as TACO1 and LRPPRC could be identified (Sasarman et al., 2010; Weraarpachai et al., 2009), they await further biochemical characterization. Therefore, the main focus in the following section is the yeast cytochrome $c$ oxidase.

Two translational activators control Cox 1 translation in yeast. Pet309 binding to COX1 mRNA is required for mRNA stability and translation (Manthey and McEwen, 1995; Tavares-Carreon et al., 2008). Mss51 can either bind COX1 mRNA or unassembled Cox1 protein. Binding of Mss51 to COX1 mRNA promotes translation. In contrast, unassembled Cox1 sequesters Mss51 rendering it incapable to initiate further COX1 translation (Decoster et al., 1990; Perez-Martinez et al., 2003). This regulatory mechanism of Cox1 synthesis prevents accumulation of unassembled Cox1 in the inner membrane, which otherwise can lead to generation of reactive oxygen species (Khalimonchuk et al., 2007).

The 12 transmembrane helices of Cox 1 are cotranslationally inserted into the inner membrane by Oxa1. Furthermore the insertion of the heme and copper cofactors into Cox1 is also believed to occur cotranslationally (Carr and Winge, 2003; Khalimonchuk and Rödel, 2005). After synthesis, Cox 1 is bound by the assembly factors Cox14 and Coa3, which recruit Mss51 (Barrientos et al., 2004; Fontanesi et al., 2011; Mick et al., 2010). This complex is subsequently bound by Coa1, a factor suggested to be involved in copper insertion into Cox 1 and required for translational regulation of COX1 mRNA (Mick et al., 2007; Pierrel et al., 2007). The complex, consisting of Cox1, Cox14, Coa1, Coa3 and Mss51 is essential for feed back regulation of Cox1 expression (Fig 1.4), since Mss51 can not induce translation in this complex (Mick et al., 2010; Perez-Martinez et al., 2003). Interestingly this complex accumulates in cells lacking Shyl and very little assembled mature cytochrome $c$ oxidase can be detected (Mick et al., 2010). Although the mechanism remains unclear, Shy1 is required for release of Mss51, which then activates further Cox1 expression. Shy1 is also 


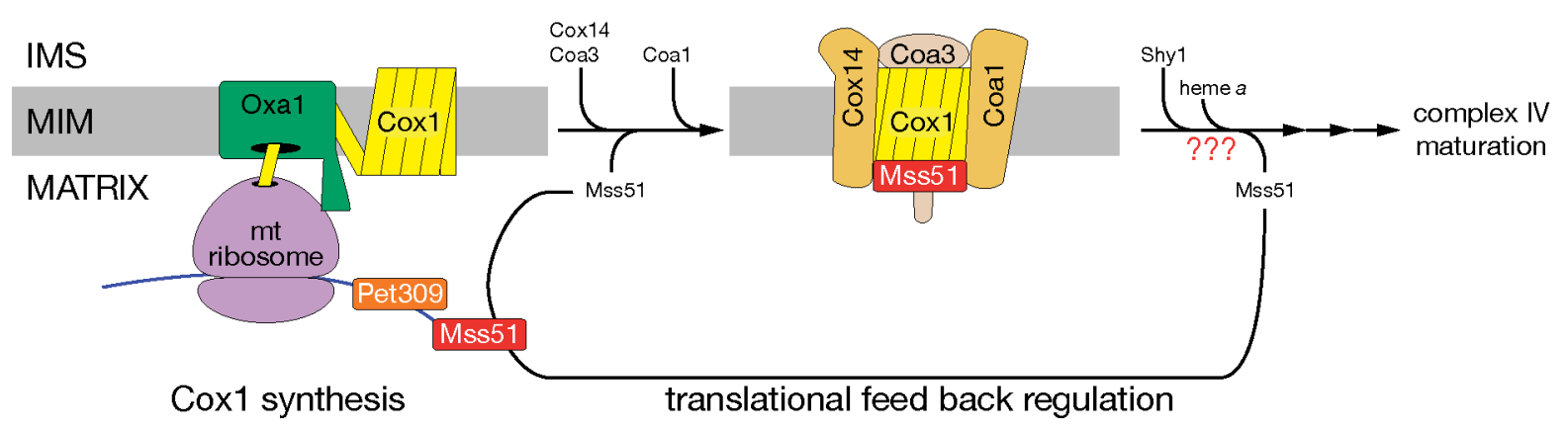

Figure 1.4 Translational feed back regulation of Cox1 synthesis in yeast

Cox1 is synthesized dependent on the translational activators Mss51 and Pet309 and cotranslationally inserted into the inner membrane by the OXA-machinery. Subsequently the translational activator Mss51 forms a complex with Cox1, Cox14, Coa1 and Coa3, and thereby cannot promote further Cox1 synthesis. Dependent on Shyl cytochrome $c$ oxidase assembly proceeds, Mss51 is released and can initiate additional Cox1 synthesis.

required for initiation of subsequent steps of cytochrome $c$ oxidase assembly, which might involve insertion of heme into Cox1 (see section 1.5.1).

Little is known about the subsequent steps of cytochrome $c$ oxidase assembly. However, pulse-chase labeling of mitochondria-encoded subunits in cultured human cells revealed subassemblies or assembly intermediates (Nijtmans et al., 1998). In addition it was shown that assembly intermediates accumulate if assembly factors are mutated in patients or knocked down in cultured cells (Horan et al., 2005; Oswald et al., 2009; Stiburek et al., 2005; Williams et al., 2004). Hence, a stepwise, assembly line like biogenesis of the cytochrome $c$ oxidase has been proposed (Nijtmans et al., 1998). This assembly line model has been refined since then and current models (Herrmann and Funes, 2005; Mick et al., 2011) suggest association of Cox 5 and Cox6 to Cox 1 before the other mitochondria-encoded subunits (Cox 2 and Cox 3 ) and Cox 4 associate to the complex. Subsequently, a preformed complex of Cox7, Cox 8 and Cox9 is incorporated. Finally Cox 12 and Cox13 are assembled which are not essential for enzymatic activity. 


\subsection{Mitochondrial diseases}

\subsubsection{Mitochondrial dysfunction leads to multisystem disorders}

Although mitochondrial disorders were initially considered to be very rare, recent estimations on the prevalence of mitochondrial disorders vary between 1:5000 and 1:10000 (McFarland et al., 2002; Schaefer et al., 2004).

Mitochondria play a central role in bioenergetics of eukaryotic cells. Therefore mitochondrial dysfunction especially affects highly energy-dependent tissues and organs, such as heart, the central nervous system and skeletal muscle (Fig 1.5A). Although genetically very diverse, mitochondrial disorders share a common set of clinical symptoms. Characteristic clinical features include specific abnormalities of the brain and nervous system such as blindness, deafness and mental retardation. Moreover, distinct biochemical findings such as accumulation of lactate in the blood or absence of enzyme activities (e.g. cytochrome $c$ oxidase, pyruvate dehydrogenase) are typical for mitochondrial disorders.

Mitochondrial diseases can be classified dependent on the clinical manifestation. Some of these disorders only show one clinical feature. LHON (Leber hereditary optic neuropathy) for example is characterized by development of blindness in otherwise healthy young adults (YuWai-Man et al., 2009). Most mitochondrial diseases however display a combination of several clinical features and most patients show multiple symptoms in various tissues. The Leigh Syndrome (LS) and the Mitochondrial DNA Depletion Syndrome (MDDS) are examples for disorders with multiple clinical manifestations. The multitude of symptoms in affected patients is the reason for the severe progression of mitochondrial diseases and strongly reduced lifespan.

The clinical manifestations and classification of mitochondrial diseases are reviewed in detail elsewhere (DiMauro and Schon, 2008; Finsterer, 2004; Zeviani and Carelli, 2007).

\subsubsection{Mutations leading to mitochondrial diseases}

Due to the dual genetic origin of mitochondrial proteins, mitochondrial diseases can be caused by mutations of the mitochondrial or nuclear genome. 

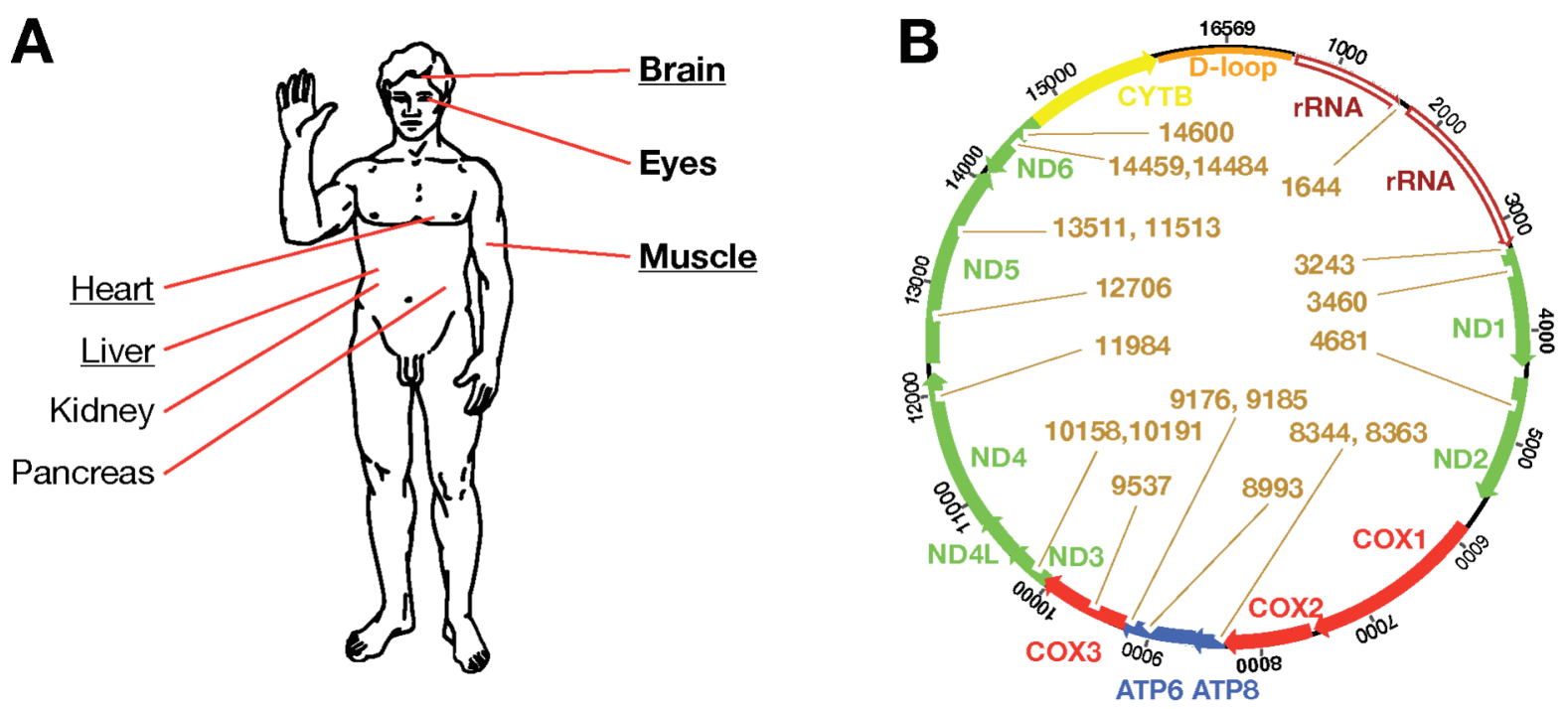

Figure 1.5 Heterogeneity of mitochondrial diseases

(A) Mitochondrial disorders have an effect on multiple organs and tissues, as depicted. Organs, affected in Leigh Syndrome (bold) and in the Mitochondrial DNA Depletion Syndrome (underlined) are indicated. (B) Organization of the human mitochondrial genome, encoding for subunits of complex I (green), III (yellow), IV (red) and the $\mathrm{F}_{1} \mathrm{~F}_{\mathrm{O}}$-ATPase (blue). As an example for the multitude of pathogenic mitochondrial DNA point mutations, bases affected in Leigh Syndrome patients as reviewed in Finsterer (2008) are indicated (brown).

The proteins encoded by the human mitochondrial DNA are subunits of the respiratory chain (Fig 1.5B). Hence, mutations of the mitochondrial genome affect respiratory chain biogenesis and function. Owing to the maternal inheritance of the mitochondrial genome, pathogenic mutations are also inherited maternally. Although mitochondrial DNA and the maternal inheritance of many mitochondrial diseases were known for a long time, the first mitochondrial DNA mutations, leading to disease, were identified not before 1988 (Holt et al., 1988; Wallace et al., 1988). Subsequently, the number of identified patient mutations increased rapidly and now more than 200 pathogenic point mutations and countless deleterious deletions and rearrangements have been identified (DiMauro and Schon, 2008).

For the severity of dysfunction due to mitochondrial DNA mutations it is critical if all mitochondrial DNA molecules within a cell are similar (homoplasmy) or if there is a mixture of different alleles of mitochondrial DNA molecules (heteroplasmy). Due to the central role of mitochondria in energy metabolism it is believed that most homoplasmic mitochondrial DNA mutations are lethal. In fact only very few homoplasmic mutations were described in patients. The effect of heteroplasmic mitochondrial point mutations strongly depends on the proportion of mutated DNA molecules. Usually mitochondrial function is only impaired if 
the mutant load is above a critical value (threshold), which is typically above $70-80 \%$ dependent on the mutation. Heteroplasmy and the threshold effect of mitochondrial DNA mutations account for the remarkable heterogeneity of mitochondrial DNA related diseases. Often there is a correlation of the severity of the disease and the mutant load of the mitochondrial point mutation. An example is the $T^{8993} \mathrm{G}$ mutation of the mitochondrial genome, which causes the Leigh Syndrome (see below) at 90\% mutant load or the much less severe NARP syndrome at 70-80\% mutant load (Holt et al., 1990; Tatuch et al., 1992).

In contrast to mitochondrial DNA mutations, nuclear mutations causing mitochondrial diseases affect multiple mitochondrial activities and functions. First, respiratory chain function can be affected by mutations of nuclear-encoded respiratory chain subunits or assembly factors. In addition, the Krebs cycle and the fatty acid metabolism is often impaired due to nuclear gene mutations (Pithukpakorn, 2005; Rinaldo et al., 2002). Defects in metabolic functions of mitochondria can be either caused by mutation of functional enzymes or metabolite transporters. Besides metabolic defects, also impaired mitochondrial biogenesis can lead to disease. In case of the Barth Syndrome (Barth et al., 1983), the synthesis of cardiolipin, a characteristic phospholipid of the mitochondrial inner membrane, is impaired (Vreken et al., 2000; Xu et al., 2006). In addition, mitochondrial biogenesis can be affected by mutations of components of the mitochondrial import machinery such as TIMM8A or HSPD1 (Hansen et al., 2002; Roesch et al., 2002). Furthermore, mitochondrial morphology and dynamics can be impaired by nuclear gene mutations as shown for mutations affecting OPA1, a factor involved in mitochondrial fusion (Alexander et al., 2000; Delettre et al., 2000).

Another class of nuclear gene mutations affects the maintenance or expression of the mitochondrial genome. Mutations affecting the mitochondrial replication machinery lead to depletion of mitochondrial DNA (see section 1.4.4). Additionally, mutations of ribosomal subunits, such as MRPS16 (Miller et al., 2004), as well as translation elongation factors (Smeitink et al., 2006; Valente et al., 2007) have been identified in patients leading to impaired mitochondrial translation.

Taken together, nuclear gene mutations affect various mitochondrial functions. However, the effects of these heterogeneous mutations often lead to similar clinical features due to general energy depletion. The heterogeneity of causes for similar clinical symptoms is a major complication for the development of specific treatments for mitochondrial diseases. Although 
the clinical symptoms are described in detail and many pathogenic mutations are identified, the molecular etiology and the relation of the mutations and the pathogenesis of most mitochondrial diseases remain still enigmatic.

\subsubsection{Leigh Syndrome}

Leigh Syndrome (LS), a severe neurodegenerative disorder of infancy, was first described in 1951 (Leigh, 1951). LS is believed to be the most common mitochondrial disorder of infancy with a prevalence of 1:40000 (Rahman et al., 1996). Characteristic for this disease are symmetrical necrotic lesions in the brain (cerebellum, brainstem, diencephalon). Typical clinical features of LS include mental retardation, loss of motor skills, general weakness and epileptic seizures (Finsterer, 2008; Rahman et al., 1996). In general LS manifests within the first year of life and most patients die within six years due to their severe neurological problems.

Biochemically LS is characterized by respiratory chain defects or in some cases defects of the pyruvate dehydrogenase complex. Although LS is clinically well defined an enormous number of different mutations in nuclear and mitochondrial (Fig 1.5B) genes have been found in patients (Finsterer, 2008). For instance mutations in all the 14 core subunits of complex I, both nuclear and mitochondria-encoded, have been described to cause LS. Moreover, also mutations affecting complex II, complex IV or the $\mathrm{F}_{1} \mathrm{~F}_{\mathrm{O}}$-ATPase can lead to LS. It should also be noted that not only mutations of structural subunits but also assembly factors of respiratory chain complexes can cause LS.

Although several respiratory chain complexes can be affected, cytochrome $c$ oxidase deficiency is the most common reason for LS (Pequignot et al., 2001). Because complete loss of cytochrome $c$ oxidase function is believed to be lethal, in affected LS patients a residual cytochrome $c$ oxidase activity of 10-50 \% can be determined (Brown and Brown, 1996; Merante et al., 1993).

Leigh Syndrome with isolated cytochrome $c$ oxidase deficiency is most commonly caused by SURF1 mutations, first described by Tiranti et al. (1998). To date, numerous different SURF1 mutations have been described in LS-patients (Pequignot et al., 2001), most of which are deletions, insertions and mutations of splicing sites leading to alterations of the coding sequence and absence of the SURF1 protein. In addition, most point mutations described so far introduce stop codons and only few missense mutations were identified (see table 1.1). 
Patient missense mutations that lead to exchange of conserved amino acids indicate the high functional relevance of the affected residues. Hence, the role of these residues is of special interest and might reveal the molecular function of SURF1 in more detail.

\subsubsection{Mitochondrial DNA Depletion Syndrome}

The Mitochondrial DNA Depletion Syndrome (MDDS) was first described in 1991 (Moraes et al., 1991). MDDS is characterized by a reduction of mitochondrial DNA copy number below 35\%. Interestingly, for unknown reasons, this loss of mitochondrial DNA occurs tissue specifically. Mitochondrial DNA depletion results in insufficient synthesis of respiratory chain subunits and energy deficiency. Dependent on the affected tissue, different clinical manifestations can be observed. The two most prevalent forms especially affect skeletal muscle (myopathic MDDS) or show progressive liver and brain dysfunctions (hepatocerebral MDDS). In addition mitochondrial DNA depletion also affects other tissues and organs and the clinical manifestations are heterogeneous (Spinazzola and Zeviani, 2007).

MDDS is caused by impaired mitochondrial DNA integrity. Although mitochondrial DNA replicates autonomously, the factors of the replication machinery are nuclear-encoded. Not surprising, mutations in these genes were identified in MDDS patients. Various mutations in $P O L G$, encoding the mitochondrial DNA polymerase ( $p o l \gamma)$ were described (Graziewicz et al., 2006; Hudson and Chinnery, 2006). Moreover mutations were identified affecting the mitochondrial DNA helicase Twinkle or factors involved in nucleotide transport and metabolism such as the adenine nucleotide translocator, the mitochondrial thymidine kinase and deoxyguanosine kinase (Kaukonen et al., 2000; Saada et al., 2001; Spelbrink et al., 2001). So far mutations in nine nuclear genes have been identified to cause MDDS (Copeland, 2008). Except of one, these genes encode for factors involved in mitochondrial DNA replication or nucleotide homeostasis. The exception is MPV17, which encodes a mitochondrial inner membrane protein of unknown function.

MPV17 mutations lead to severe forms of hepatocerebral MDDS, first reported by Spinazzola et al. (2006). To date 20 different mutations are described, most of which are missense mutations (see table 1.1) or short in frame deletions affecting one to three amino acids (ElHattab et al., 2010).

MPV17 is highly conserved in eukaryotes and also has peroxisomal paralogs. Although studies on mammalian and yeast members of this protein family have been performed, 
MPV17 function remains unresolved. Therefore it is unknown how MPV17 dysfunction results in mitochondrial DNA depletion.

\subsection{Yeast as a model for mitochondrial diseases}

Yeast cannot only be considered to be one of "humans best friends", since it has been used for millennia in brewing and baking processes (Samuel, 1996; Sicard and Legras, 2011), it has also been used to study biochemical processes since the $19^{\text {th }}$ century (Barnett, 1998, 2000). In the last decades advances in molecular biology revealed the extraordinary conservation of basal biochemical processes in all eukaryotes. Hence, yeast is an ideal model to study eukaryotic biochemistry. Interestingly, most of the current knowledge of the molecular basis of human mitochondrial function is derived from studies on the yeast Saccharomyces cerevisiae as a model. The facultative anaerobic character of S. cerevisiae allows analysis of respiratory chain dysfunction. Furthermore, the compact genome and the well established genetic manipulation techniques make $S$. cerevisiae an ideal model to study mitochondrial biogenesis and function.

Many human genes associated with mitochondrial diseases have yeast counterparts (Barrientos, 2003) that can be used to study the molecular etiology of these diseases. In this regard patient point mutations that affect amino acids also conserved in $S$. cerevisiae are most interesting since they indicate a functional importance of these amino acids. Amino acid exchanges due to these point mutations can be mimicked in the yeast protein. In this study, point mutations affecting SURF1 and MPV17, identified in patients of Leigh Syndrome and Mitochondrial DNA Depletion Syndrome respectively, were analyzed using the corresponding yeast proteins as a model. 
1. Introduction

Table 1.1 Pathogenic missense mutations affecting SURF1 and MPV17

\begin{tabular}{|c|c|c|}
\hline Amino acid exchanges in SURF1 & Reference & Conservation in yeast ${ }^{\mathrm{a}}$ \\
\hline $\mathrm{Y}^{274} \mathrm{D}$ & Teraoka et al. (1999) & $+\left(\mathrm{Y}^{344}\right)$ \\
\hline $\mathrm{G}^{124} \mathrm{R}$ & Coenen et al. (1999) & $+\left(G^{137}\right)$ \\
\hline $\mathrm{G}^{124} \mathrm{E}$ & Poyau et al. (2000) & $+\left(G^{137}\right)$ \\
\hline $\mathrm{I}^{246} \mathrm{~T}$ & Poyau et al. (2000) & $+/-\left(\mathrm{F}^{249}\right)$ \\
\hline $\mathrm{L}^{203} \mathrm{P}$ & Sacconi et al. (2003) & $+/-\left(V^{205}\right)$ \\
\hline $\mathrm{Q}^{80} \mathrm{R}$ & Coenen et al. (2006) & $+\left(Q^{91}\right)$ \\
\hline $\mathrm{V}^{177} \mathrm{G}$ & Yuksel et al. (2006) & $+/-\left(\mathrm{I}^{175}\right)$ \\
\hline $\mathrm{R}^{192} \mathrm{G}$ & Yang et al. (2006) & $+\left(\mathrm{R}^{190}\right)$ \\
\hline $\mathrm{D}^{202} \mathrm{H}$ & Yang et al. (2006) & $-\left(K^{204}\right)$ \\
\hline $\mathrm{R}^{192} \mathrm{~W}$ & Piekutowska-Abramczuk et al. (2009) & $+\left(\mathrm{R}^{190}\right)$ \\
\hline $\mathrm{M}^{235} \mathrm{~T}$ & Piekutowska-Abramczuk et al. (2009) & $+\left(\mathrm{M}^{238}\right)$ \\
\hline $\mathrm{Y}^{274} \mathrm{C}$ & Piekutowska-Abramczuk et al. (2009) & $+\left(\mathrm{Y}^{344}\right)$ \\
\hline Amino acid exchanges in MPV17 & Reference & Conservation in yeast $\mathrm{t}^{\mathrm{a}}$ \\
\hline $\mathrm{R}^{50} \mathrm{Q}$ & Spinazzola et al. (2006) & $+\left(\mathrm{R}^{51}\right)$ \\
\hline $\mathrm{R}^{50} \mathrm{~W}$ & Spinazzola et al. (2006) & $+\left(\mathrm{R}^{51}\right)$ \\
\hline $\mathrm{N}^{166} \mathrm{~K}$ & Spinazzola et al. (2006) & $+\left(\mathrm{N}^{172}\right)$ \\
\hline $\mathrm{G}^{24} \mathrm{~W}$ & Spinazzola et al. (2008) & $+\left(G^{24}\right)$ \\
\hline$S^{170} F$ & Kaji et al. (2009) & $+\left(S^{176}\right)$ \\
\hline $\mathrm{K}^{88} \mathrm{E}$ & El-Hattab et al. (2010) & $+/-\left(\mathrm{R}^{94}\right)$ \\
\hline $\mathrm{G}^{94} \mathrm{R}$ & El-Hattab et al. (2010) & $-\left(L^{100}\right)$ \\
\hline $\mathrm{P}^{98} \mathrm{~L}$ & El-Hattab et al. (2010) & $+\left(\mathrm{P}^{104}\right)$ \\
\hline $\mathrm{A}^{162} \mathrm{D}$ & El-Hattab et al. (2010) & $+\left(\mathrm{A}^{168}\right)$ \\
\hline
\end{tabular}

${ }^{\mathrm{a}}$ Corresponding conserved (+), similar (+/-) and non conserved (-) amino acids are given in brackets. 


\subsubsection{Shy1 - a model for SURF1 associated LS}

Mutations affecting SURF1 are the most prevalent cause of Leigh Syndrome with isolated cytochrome $c$ oxidase deficiency. SURF1 is encoded by the first of six genes of the surfeitlocus, a cluster of unrelated housekeeping genes (Duhig et al., 1998). SURF1 is highly conserved in respiring organisms. Most insights into the function of SURF1 came from studies on its yeast homolog Shy1. Initially SHY1 mutations were identified in pet mutants by Mashkevich et al. (1997) indicating that Shy1 is required for respiratory competence. In the same study it was shown that Shyl localizes to the mitochondrial inner membrane. In fact transmembrane span predictions and protease protection analyses indicate that SURF1/Shy1 contains a large inter membrane space domain and is inserted into the inner membrane with $\mathrm{N}$ - and C-terminal helices. The reduced cytochrome $c$ oxidase content in cells lacking Shy 1 (Mashkevich et al., 1997) was characterized by Nijtmans et al. (2001) indicating that in shy $1 \Delta$ cells the assembly of the cytochrome $c$ oxidase is strongly reduced. However, a residual cytochrome $c$ oxidase activity of $\sim 30 \%$ was determined, apparently not sufficient for respiratory growth. The defective cytochrome $c$ oxidase assembly in shyld cells was explained by studies of Barrientos et al. (2002) demonstrating that Shy1 is required for Cox1 expression. In addition, it was shown that mutations in MSS51 suppress a deletion of SHY1. Protein interaction analyses by Mick et al. (2010; 2007) revealed physical interactions of Shy1 with various Cox1 translational regulators such as Mss51, Coa1, Coa3 and Cox14, that are involved in translational feed-back regulation of Cox1. In addition, the assembly of Shy1 into high molecular weight complexes, possible Cox1 containing cytochrome $c$ oxidase assembly intermediates was demonstrated. Interestingly, in fibroblasts from patients with SURF1 associated Leigh Syndrome, an accumulation of cytochrome $c$ oxidase assembly intermediates could also be detected (Stiburek et al., 2005; Williams et al., 2004). Moreover in yeast, Shy1 association to the mature cytochrome $c$ oxidase was shown (Mick et al., 2007). However, a possible role of this association in repair processes or late assembly steps remains speculative. Although a role of Shy1/SURF1 in Cox1 expression and cytochrome $c$ oxidase assembly is evident, its exact molecular function is still elusive.

Interestingly, studies on bacterial Shy1/SURF1 homologs revealed its potential role in heme insertion into Cox1. Smith et al. (2005) showed that Rhodobacter sphaeroides lacking Surf1 still assemble an $a a_{3}$-type cytochrome $c$ oxidase. However, the activity of this cytochrome $c$ oxidase was reduced to $35 \%$ and heme $a_{3}$ was missing in about $50 \%$ of the enzyme 
complexes. This finding was verified using Paracoccus denitrificans Surf1, which was shown to bind heme a in vitro (Bundschuh et al., 2009; Bundschuh et al., 2008)

In yeast, although indication exists that heme insertion into Cox1 takes place in a Shy1 dependent step of assembly (Khalimonchuk et al., 2010; Khalimonchuk et al., 2007) the molecular mechanism of heme insertion is unresolved.

Interestingly, SURF1 point mutations have been identified in LS patients affecting highly conserved amino acids (see table 1.1). In the course of the present work Bestwick et al. (2010a) reported the analysis of three patient point mutations in SURF1, characterized using the correspondent SHY1 mutations. However, although in some cases loss of functionality due to the mutation could be shown, the molecular basis of protein dysfunction remains to be clarified.

\subsubsection{Sym1 - a model for MPV17, a protein involved in MDDS}

The Mitochondrial DNA Depletion Syndrome (MDDS) is caused by mutations of factors involved in mitochondrial DNA replication or MPV17, a protein of unknown function. The MPV17 gene was named after the Mpv17 mouse mutation, generated by random integration of provirus DNA into the mouse genome (Weiher et al., 1990). Homozygous MPV17 ${ }^{-/-}$ mutant mice showed progressive kidney dysfunction and were suggested to be an ideal model for kidney diseases. Although MPV17 was initially suggested to be a peroxisomal protein (Zwacka et al., 1994), studies on the yeast MPV17 homolog, termed Sym1 (stress inducible

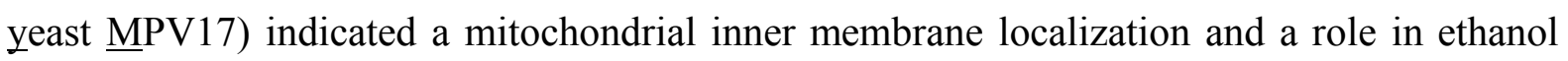
and heat tolerance (Trott and Morano, 2004). The role of MPV17 in the Mitochondrial DNA Depletion Syndrome was reported by Spinazzola et al. (2006), also confirming its mitochondrial inner membrane localization. Although to date multiple MPV17 mutations have been described in MDDS patients (El-Hattab et al., 2010), the molecular function of MPV17 is still unknown.

Interestingly, also peroxisomal paralogs of MPV17/Sym1, termed PMP22 or Yor292c in mammals or yeast respectively, could be identified. Studies on PMP22 indicated a pore forming activity of this protein (Rokka et al., 2009; Van Veldhoven et al., 1987).

It has been speculated that the MPV17/Sym1 protein family plays a role in the metabolism of reactive oxygen species. However different studies came to contradictory results (Trott and Morano, 2004; Wagner et al., 2001; Zwacka et al., 1994) and the role of MPV17/Sym1 in 
oxidative stress remains elusive. Additionally, recent studies on Sym1 suggested a role in energy metabolism and mitochondrial morphology (Dallabona et al., 2010). However, an indirect effect due to loss of mitochondrial DNA as previously reported (Spinazzola et al., 2006) could not be excluded.

The biogenesis of MPV17 and Sym1 in mitochondria and its molecular function remains unknown. Hence, the mechanism that leads to disease in patients with MPV17 associated MDDS is unclear. Yeast might be an ideal model to unravel the biogenesis, interactions and molecular functions of the mitochondrial members of the MPV17/Sym1 family. Furthermore, the use of corresponding yeast mutations might help to understand how the patient MPV17 mutations result in loss of mitochondrial DNA. 


\subsection{Aim of this work}

Mitochondrial dysfunctions are severe multisystem disorders, mostly caused by energy depletion due to mutations in the mitochondrial or nuclear genome. The few mitochondriaencoded proteins and their dysfunctions are studied and characterized very well. In contrast, the function of many nuclear-encoded mitochondrial proteins, involved in human diseases, is still ill-defined. Accordingly, the molecular etiology of mutations affecting these proteins is unresolved. In this work, two nuclear-encoded proteins (SURF1 and MPV17) involved in mitochondrial diseases were studied in detail.

SURF1, associated with Leigh Syndrome, is an assembly factor of the cytochrome $c$ oxidase with unclear molecular function. In patients, mutations affecting SURF1 lead to defective cytochrome $c$ oxidase and severe neurodegenerative disorders. Although most patients show deletions, nonsense mutations and mutations of splicing sites, a few point mutations have been identified. The first point mutations described affect the highly conserved amino acids $\mathrm{G}^{124}$ and $\mathrm{Y}^{274}$, which were analyzed in this study.

MPV17 mutations have been identified in patients of the Mitochondrial DNA Depletion Syndrome. The function of MPV17, the mitochondrial member of a big family of homolog proteins is not known. Therefore it remains enigmatic how mutant MPV17 leads to loss of mitochondrial DNA. The first MPV17 mutations found in patients affect the highly conserved amino acids $\mathrm{R}^{50}$ and $\mathrm{N}^{166}$. The effect of these mutations as well as the function and biogenesis of the wild-type protein is analyzed in this study.

The significance for diseases and the high conservation of amino acids in SURF1 and MPV17, affected by patient point mutations, indicate the functional importance of these residues. This study aims to resolve the molecular function of SURF1 and MPV17 by characterization of these amino acid exchanges and thereby to understand mechanisms that lead to mitochondrial disorders.

This work describes the development of yeast models for patient point mutations that were used for phenotypical and biochemical characterization. With these models the effect of the patient mutations on protein biogenesis, stability and function was analyzed. Furthermore the yeast homolog proteins were used to biochemically characterize the molecular function of wild-type SURF1 and MPV17. 


\section{Materials and Methods}

\subsection{Materials}

\subsubsection{Chemicals, enzymes and kit systems}

Standard chemicals were used in analytical grade quality and obtained from AppliChem (Darmstadt), Merck (Darmstadt), Serva (Heidelberg), Sigma Aldrich (Taufkirchen) or Roth (Karlsruhe). Restriction enzymes were ordered from Fermentas (St. Leon-Rot). Deoxyoligonucleotides were obtained from Metabion (Martinsried) and used as primers for PCR or site-directed mutagenesis. Special chemicals and enzymes are listed in table 2.1 and 2.2. Kit systems (listed in table 2.3) were used according to the manufacturer's recommendations.

\subsubsection{Microorganisms and cell lines}

Escherichia coli (E. coli) strain XL1-blue (Stratagene) was used for molecular cloning. Genotypes of Saccharomyces cerevisiae (S. cerevisiae) strains used in this study are listed in table 2.4. For human cell culture HEK $293 \mathrm{~T}$ cells (ATCC, CRL-11268), adherent, immortalized human embryonic kidney cells were used.

\subsubsection{Plasmids}

All plasmids used in this study are listed in table 2.5. Plasmids were generated as described in section 2.5.5 and propagated in E. coli XL1-blue cells.

\subsubsection{Antibodies}

Primary polyclonal antibodies were raised in rabbit, immunized either with peptides or purified proteins (Gramsch Laboratories, Schwabhausen). Secondary antibodies against rabbit immunoglobulin, coupled to horseradish-peroxidase were used for Western blot detection. Most important antibodies used for Western blot analysis and coimmunoprecipitation experiments, are listed in table 2.6. 
2. Materials and Methods

Table 2.1 Special chemicals and consumables

\begin{tabular}{|c|c|c|c|}
\hline Product & Manufacturer & Product & Manufacturer \\
\hline ATP & Roche & PEG4000 & Fluka \\
\hline NADH & Roche & Herring Sperm DNA & Promega \\
\hline Creatine phosphate & Roche & PVDF membrane & Millipore \\
\hline$\left[{ }^{35} \mathrm{~S}\right]$ methionine & Hartmann-Analytic & X-Ray films & GE-Healthcare \\
\hline $\begin{array}{l}\text { DNA-Marker: } \\
\text { GeneRuler }\end{array}$ & Fermentas & $\begin{array}{l}\text { ECL Western blotting } \\
\text { detection reagents }\end{array}$ & GE-Healthcare \\
\hline $\begin{array}{l}\text { BN-Marker: HMW } \\
\text { calibration kit }\end{array}$ & GE-Healthcare & $\begin{array}{l}\text { MitoTracker Orange } \\
\text { CMTMRos }\end{array}$ & Invitrogen \\
\hline $\begin{array}{l}\text { SDS-Marker: Broad } \\
\text { Range }\end{array}$ & Biorad & Rapamycin & LC Laboratories \\
\hline IgG (human) & Sigma Aldrich & AntimycinA & Sigma Aldrich \\
\hline IgG (bovine) & Biorad & Oligomycin & Sigma Aldrich \\
\hline Roti-Quant & Roth & Valinomycin & Calbiochem \\
\hline Digitonin & Calbiochem & Chloramphenicol & Serva \\
\hline Triton X-100 & Serva & Cycloheximide & Sigma Aldrich \\
\hline Complete EDTA free & Roche & ProteinA-Sepharose & GE-Healthcare \\
\hline PMSF & Sigma Aldrich & Ni-NTA-Agarose & Qiagen \\
\hline
\end{tabular}


Table 2.2 Enzymes used in this study

\begin{tabular}{lll}
\hline Enzyme & Manufacturer & Application \\
\hline TEV-Protease & Invitrogen & Protein purification \\
KOD Hot start DNA Polymerase & Novagen & PCR \\
Trypsin-EDTA & Gibco & Detaching of cultured cells \\
Creatin Kinase & Roche & In vitro import \\
Proteinase K & Roche & Protease protection assays \\
Zymolyase $20 \mathrm{~T}$ & Seikagaku & Spheroplasting of yeast cells
\end{tabular}

Table 2.3 Kit systems used in this study

\begin{tabular}{|c|c|c|}
\hline Kit & Manufacturer & Application \\
\hline $\begin{array}{l}\text { Wizard Plus SV Minipreps DNA } \\
\text { Purification System }\end{array}$ & Promega & Plasmid miniprep \\
\hline NucleoBond PC 100 & Macherey-Nagel & Plasmid midiprep \\
\hline $\begin{array}{l}\text { Wizard SV Gel and PCR Clean- } \\
\text { Up System }\end{array}$ & Promega & DNA fragment purification \\
\hline $\begin{array}{l}\text { High Pure PCR Template } \\
\text { Preparation Kit }\end{array}$ & Roche & $\begin{array}{l}\text { Isolation of highly purified yeast } \\
\text { genomic DNA }\end{array}$ \\
\hline Rapid DNA Ligation Kit & Fermentas & Ligation of DNA fragments \\
\hline $\begin{array}{l}\text { QuikChange Site-Directed } \\
\text { Mutagenesis Kit }\end{array}$ & Agilent & $\begin{array}{l}\text { Site directed mutagenesis of } \\
\text { plasmids }\end{array}$ \\
\hline $\begin{array}{l}\text { BigDye Terminator v1.1 Cycle } \\
\text { Sequencing Kit }\end{array}$ & Applied Biosystems & DNA sequencing \\
\hline mMESSAGE mMACHINE SP6 & Ambion & In vitro transcription \\
\hline MEGAclear & Ambion & Purification of RNA \\
\hline $\begin{array}{l}\text { Flexi Rabbit Reticulocyte Lysate } \\
\text { System }\end{array}$ & Promega & In vitro translation \\
\hline $\begin{array}{l}\text { TNT SP6 Quick Coupled } \\
\text { Transcription/Translation System }\end{array}$ & Promega & $\begin{array}{l}\text { Coupled in vitro } \\
\text { transcription/translation }\end{array}$ \\
\hline
\end{tabular}


Table 2.4 Yeast strains used in this study

\begin{tabular}{|c|c|c|}
\hline S. cerevisiae strain & Genotype & Reference \\
\hline YPH499 & $\begin{array}{l}\text { MATa ade2-101 his3- } \Delta 200 \text { leu2- } \Delta 1 \text { lys } 2-801 \\
\text { trp1- } \triangle 63 \text { ura3-52 }\end{array}$ & Sikorski and Hieter (1989) \\
\hline DaMY21 (coal $\Delta)$ & $\begin{array}{l}\text { MATa ade2-101 his } 3-\Delta 200 \text { leu2- } \Delta 1 \text { lys } 2-801 \\
\text { trp1- } \triangle 63 \text { ura3-52 coa1::HisMX6 }\end{array}$ & Mick et al. (2007) \\
\hline 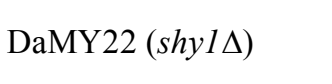 & $\begin{array}{l}\text { MATa ade2-101 his3- } \Delta 200 \text { leu2- } \Delta 1 \text { lys } 2-801 \\
\text { trp1- } \triangle 63 \text { ura3-52 shy1 }: \text { HisMX6 }\end{array}$ & Mick et al. (2007) \\
\hline 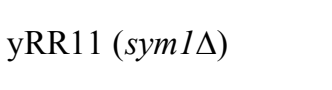 & 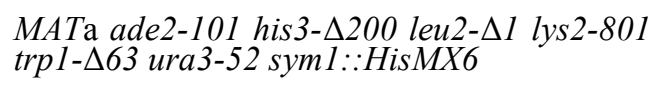 & this study \\
\hline yRR13 $\left(S Y M 1^{\text {Prot.A }}\right)$ & 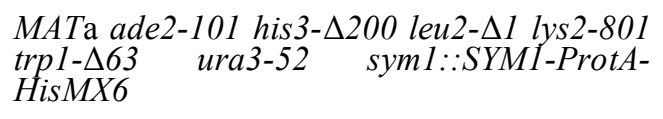 & this study \\
\hline yRR37 $\left(S Y M 1^{\mathrm{GFP}}\right)$ & 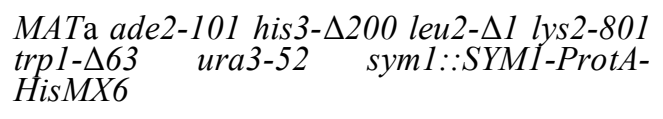 & this study \\
\hline YPH499 $\left[r h o^{0}\right]$ & 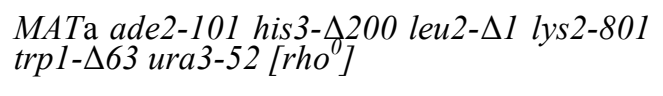 & AG Rehling \\
\hline $\begin{array}{l}\text { YPH-BG-54-1-1 } \\
(\text { tim54-11) }\end{array}$ & $\begin{array}{l}\text { MATa ade2-101 his3- } \Delta 200 \text { leu2- } \Delta 1 \text { lys2-801 } \\
\text { trp1- } \triangle 63 \text { ura3-52 tim54::tim54-11 }\end{array}$ & Wagner et al. (2008) \\
\hline $\begin{array}{l}\text { YPH-BG-tim12-4 } \\
\text { (tim 12-4) }\end{array}$ & $\begin{array}{l}\text { MATa ade2-101 his3- } \Delta 200 \text { leu2- } \Delta 1 \text { lys2-801 } \\
\text { trp1- } \triangle 63 \text { ura3-52 tim 12::ADE2 [pFL39- } \\
\text { TIM12-4ts] }\end{array}$ & Gebert et al. (2008) \\
\hline 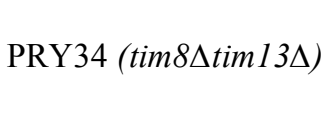 & $\begin{array}{l}\text { MATa ade2-101 his3- } \Delta 200 \text { leu2- }-1 \text { lys } 2-801 \\
\text { trp1- } \triangle 63 \quad \text { ura3-52 tim 13::kanMX4 } \\
\text { tim8::TRP1 }\end{array}$ & Truscott et al. (2002) \\
\hline GB102 (tim10-2) & 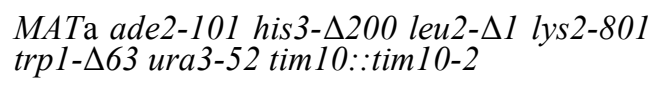 & Truscott et al. (2002) \\
\hline $\mathrm{yCS} 4(\operatorname{tim} 23 \downarrow)$ & 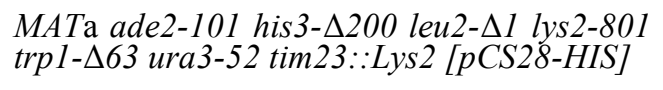 & Schulz et al. (2011) \\
\hline $\begin{array}{l}\text { YPH-BG-50-2A } \\
(\text { tim50-1) }\end{array}$ & 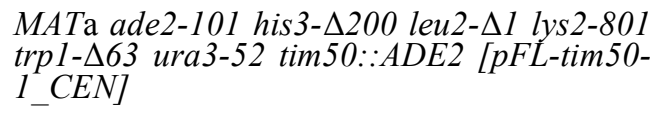 & Chacinska et al. (2005) \\
\hline BY4741 & MATa his $3-\Delta 1$ leu $2 \Delta 0$ met $15 \Delta 0$ ura $3 \Delta 0$ & Brachmann et al. (1998) \\
\hline Y05113 (uth1 1 ) & $\begin{array}{l}\text { MATa his3- } \Delta 1 \text { leu } 2 \Delta 0 \text { met15 } \Delta 0 \text { ura3 } \Delta 0 \\
\text { uth1::kanMX4 }\end{array}$ & Euroscarf \\
\hline Y02103 $(\operatorname{atg} 5 \Delta)$ & $\begin{array}{l}\text { MATa his3- } \Delta 1 \text { leu } 2 \Delta 0 \text { met15 } \Delta 0 \text { ura3 } \Delta 0 \\
\text { atg5::kanMX4 }\end{array}$ & Euroscarf \\
\hline 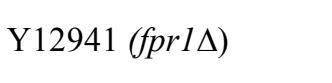 & $\begin{array}{l}\text { MATa his } 3-\Delta 1 \text { leu } 2 \Delta 0 \text { met15 } \Delta 0 \text { ura3 } \Delta 0 \\
\text { fprl::kanMX4 }\end{array}$ & Euroscarf \\
\hline
\end{tabular}


Table 2.5 Plasmids used in this study

\begin{tabular}{|c|c|c|c|}
\hline Plasmid & Backbone & Insert & Reference \\
\hline pRS416 & - & - & Stratagene \\
\hline pDaM10 (Shy $\left.1^{\mathrm{WT}}\right)$ & pGEM4Z & SHY1 & Mick et al. (2007) \\
\hline pDaM11 (Shy1 $\left.{ }^{\mathrm{G} 137 \mathrm{E}}\right)$ & pGEM4Z & SHY1-(G410A) & this study \\
\hline pDaM12 (Shy1 $\left.{ }^{\mathrm{G} 137 \mathrm{R}}\right)$ & pGEM4Z & SHY1-(G409A) & this study \\
\hline pDaM13 (Shy1 $\left.{ }^{\mathrm{Y} 34 \mathrm{D}}\right)$ & pGEM4Z & SHY1-(T1030G) & this study \\
\hline pDaM20 (Shy $\left.1^{\mathrm{WT}}\right)$ & pRS416 & $S H Y 1^{\mathrm{a}}$ & this study \\
\hline pDaM21 (Shy $\left.1^{\mathrm{G} 137 \mathrm{E}}\right)$ & pRS416 & $S H Y 1-(G 410 A)^{\mathrm{a}}$ & this study \\
\hline pDaM22 (Shy1 $\left.{ }^{\mathrm{G} 137 \mathrm{R}}\right)$ & $\mathrm{pRS} 416$ & $S H Y 1-(G 409 A)^{\mathrm{a}}$ & this study \\
\hline pDaM23 (Shy1 $\left.{ }^{\mathrm{Y} 34 \mathrm{D}}\right)$ & $\mathrm{pRS} 416$ & SHY1-(T1030G) ${ }^{\mathrm{a}}$ & this study \\
\hline pDaM24 (Shy $\left.{ }^{\mathrm{WT}} \uparrow\right)$ & $\mathrm{pRS} 426$ & $S H Y 1^{\mathrm{a}}$ & this study \\
\hline pDaM27 $\left(\right.$ Shy $\left.1^{\mathrm{Y} 344 \mathrm{D}} \uparrow\right)$ & $\mathrm{pRS} 426$ & SHY1-(T1030G) $)^{\mathrm{a}}$ & this study \\
\hline pRR7 (SURF1 $\left.{ }^{\mathrm{WT}}\right)$ & pGEM4Z & SURF1 & this study \\
\hline pRR8 (SURF1 ${ }^{\mathrm{G} 124 \mathrm{E}}$ ) & pGEM4Z & SURF1-(G371A) & this study \\
\hline pRR13 (SURF1 $\left.{ }^{\mathrm{G} 124 \mathrm{R}}\right)$ & pGEM4Z & SURF1-(G370A) & this study \\
\hline pRR14 (SURF1 $\left.{ }^{\mathrm{Y} 274 \mathrm{D}}\right)$ & pGEM4Z & $S U R F 1-(T 820 G)$ & this study \\
\hline pRR1 (Sym1 $\left.{ }^{\mathrm{WT}}\right)$ & pRS416 & $S Y M 1^{b}$ & this study \\
\hline pRR2 (Sym1 $\left.1^{\mathrm{R} 51 \mathrm{Q}}\right)$ & $\mathrm{pRS} 416$ & $S Y M 1-(A G 151 C A)^{b}$ & this study \\
\hline pRR3 $\left(\operatorname{Sym}^{\mathrm{N} 172 \mathrm{~K}}\right)$ & $\mathrm{pRS} 416$ & $S Y M 1-(C 516 A)^{b}$ & this study \\
\hline pJD27 (Sym1 $\left.{ }^{\mathrm{WT}}\right)$ & pCR-blunt-II-TOPO & $S Y M 1$ & this study \\
\hline pJD28 $\left(\mathrm{Sym}^{\mathrm{R} 51 \mathrm{Q}}\right)$ & pCR-blunt-II-TOPO & $S Y M 1-(A G 151 C A)$ & this study \\
\hline pJD30 $\left(\operatorname{Sym}^{\mathrm{N} 172 \mathrm{~K}}\right)$ & pCR-blunt-II-TOPO & $S Y M 1-(C 516 A)$ & this study \\
\hline O01 (OTC) & pGEM4 & OTC & AG Rehling (O01) \\
\hline A01 (NcAAC) & pGEM4 & $N c A A C$ & Pfanner et al. (1987) \\
\hline COX6A1 & pOTB7 & СOX6A1 & $\begin{array}{l}\text { Imagenes } \\
\text { (IRAUp969A0334D) }\end{array}$ \\
\hline
\end{tabular}

${ }^{a}$ Including $372 \mathrm{bp}$ upstream and $363 \mathrm{bp}$ downstream of the open reading frame.

${ }^{\mathrm{b}}$ Including $550 \mathrm{bp}$ upstream and $535 \mathrm{bp}$ downstream of the open reading frame. 
Table 2.6 Antibodies used in this study

\begin{tabular}{|c|c|c|}
\hline $\begin{array}{l}\text { Antibodies directed } \\
\text { against yeast proteins }\end{array}$ & Epitope, description & Reference \\
\hline$\alpha \operatorname{Sym} 1$ & C-terminal peptide, affinity purified antibody & AG Rehling (1570) \\
\hline aShy1 & Whole protein & AG Rehling (2241) \\
\hline$\alpha$ Shy $1^{\mathrm{C}}$ & C-terminal peptide, affinity purified antibody & AG Rehling (1094) \\
\hline$\alpha \operatorname{Cox} 1$ & C-terminal peptide & AG Rehling (1583) \\
\hline$\alpha \operatorname{Cox} 2$ & C-terminal peptide & AG Rehling (1948) \\
\hline$\alpha \operatorname{Cox} 4$ & Central peptide & AG Rehling (577) \\
\hline$\alpha \operatorname{Cox} 5 \mathrm{a}$ & C-terminal peptide & AG Rehling (1540) \\
\hline$\alpha$ Coa1 & C-terminal peptide & AG Rehling (1954) \\
\hline$\alpha \operatorname{Coa} 3$ & C-terminal peptide & AG Rehling (2047) \\
\hline$\alpha \operatorname{Rip} 1$ & C-terminal peptide & AG Rehling (543) \\
\hline$\alpha \mathrm{AAC}$ & Neurospora crassa protein & AG Rehling (51) \\
\hline$\alpha \operatorname{Prot} \mathrm{A}$ & Peroxidase Anti-Peroxidase & Sigma Aldrich \\
\hline $\begin{array}{l}\text { Antibodies directed } \\
\text { against human proteins }\end{array}$ & Epitope, description & Reference \\
\hline$\alpha \operatorname{SURF} 1$ & Whole protein & AG Rehling (2243) \\
\hline$\alpha \operatorname{COX} 1$ & C-terminal peptide & AG Rehling (2035) \\
\hline$\alpha \operatorname{COX} 5 \mathrm{~A}$ & C-terminal peptide & AG Rehling (2036) \\
\hline Secondary antibodies & Description & Reference \\
\hline GaR-HRP & $\alpha \operatorname{IgG(rabbit)~coupled~to~horseradish~peroxidase~}$ & Dianova \\
\hline
\end{tabular}

\subsection{Cultivation of bacteria}

E. coli cells were grown in LB medium $(0.5 \%$ yeast extract, $1 \%$ tryptone, $1 \%$ sodium chloride). Solid medium was made by addition of $1.5 \%$ agar before autoclaving. For selection of plasmids, carrying an $\mathrm{Amp}^{\mathrm{R}}$ marker $100 \mathrm{mg} / \mathrm{l}$ ampicillin was added to the medium. Liquid cultures were inoculated from a plate or 1:100-1:1000 and incubated on a shaker at $37^{\circ} \mathrm{C}$. The growth of E. coli cultures was monitored by $\mathrm{OD}_{600}$ measurements. Cryo-stocks were prepared to store E. coli strains: $1 \mathrm{ml}$ of bacterial culture was supplemented with $200 \mu 1$ of sterile $80 \%$ glycerol and stored at $-80{ }^{\circ} \mathrm{C}$. 


\subsection{Cultivation of yeast}

\subsubsection{Growth conditions and media}

S. cerevisiae strains were grown in rich medium, supplemented with $2 \%$ glucose (YPD) or $3 \%$ glycerol (YPG) as a fermentable or nonfermentable carbon source, respectively. Synthetic medium (SD, SGal, SG) was used for selection of genetic markers or for cultures that were used for in vivo labeling of mitochondrial translation products and for fluorescence microscopy. All components of the yeast media used in this study are listed in table 2.7. To obtain solid medium, $2 \%$ agar was added to the medium prior to sterilization and preparation of the plates.

Liquid yeast cultures were inoculated from a plate or 1:20 to 1:50 in order to get an appropriate cell density. Cell density was monitored by $\mathrm{OD}_{600}$ measurements $\left(\mathrm{OD}_{600}\right.$ of $1 \sim 10^{7}$ cells per ml). Cultures were shaken at $140 \mathrm{rpm}$ and incubated at $30^{\circ} \mathrm{C}$ if not indicated otherwise. For induction of autophagy, plates were supplemented with $0.2 \mu \mathrm{g} / \mathrm{ml}$ rapamycin.

Table 2.7 Media for yeast culture

\begin{tabular}{ll}
\hline Medium & Components \\
\hline YPD & $1 \%$ yeast extract, $2 \%$ peptone, $2 \%$ glucose \\
YPG & $1 \%$ yeast extract, $2 \%$ peptone, $3 \%$ glycerol \\
YPE & $1 \%$ yeast extract, $2 \%$ peptone, $3 \%$ ethanol \\
SD-Ura & $0.67 \%$ yeast nitrogen base, $0.07 \%$ CSM-Ura, $2 \%$ glucose \\
SD-His & $0.67 \%$ yeast nitrogen base, $0.07 \%$ CSM-His, $2 \%$ glucose \\
SGal-Ura & $0.67 \%$ yeast nitrogen base, $0.07 \%$ CSM-Ura, $2 \%$ galactose \\
SG-Ura & $0.67 \%$ yeast nitrogen base, $0.07 \%$ CSM-Ura, $3 \%$ glycerol \\
SE-Ura & $0.67 \%$ yeast nitrogen base, $0.07 \%$ CSM-Ura, $3 \%$ ethanol \\
SGal & $0.67 \%$ yeast nitrogen base, $0.07 \%$ CSM-Ura, 20 mg $/ 1$ uracil, $\%$ galactose \\
SG & $0.67 \%$ yeast nitrogen base, $0.07 \%$ CSM-Ura, 20 mg $/ 1$ uracil, $3 \%$ glycerol \\
\hline
\end{tabular}




\subsubsection{Yeast cryo-stocks}

To store yeast strains, $0.75 \mathrm{ml}$ of a yeast culture was supplemented with $0.75 \mathrm{ml}$ of sterile $30 \%$ glycerol and stored at $-80{ }^{\circ} \mathrm{C}$.

\subsubsection{Growth test of yeast strains}

Serial 10-fold dilutions of over night yeast cultures in YPD or SD-Ura were spotted on solid media plates, incubated for 3 to 5 days at indicated temperatures and growth was documented using a CCD camera (LAS 3000, Fujifilm).

\subsection{Culture of human cell-lines}

Human cells (HEK 293T) were cultured in Dulbecco's Modified Eagle Medium (Gibco) supplemented with $10 \%$ fetal bovine serum. Culturing was performed at $37{ }^{\circ} \mathrm{C}$ under $5 \% \mathrm{CO}_{2}$ atmosphere in $75 \mathrm{~cm}^{2}$ bottles or $175 \mathrm{~cm}^{2}$ dishes (Nunc). Prior to confluency, the culture was split 1:10 by detaching the cells from the surface using trypsin-EDTA (Gibco). Cells were washed with PBS (137 mM sodium chloride, $2.7 \mathrm{mM}$ potassium chloride, $12 \mathrm{mM}$ potassium phosphate, $\mathrm{pH} 7.4$ ), sedimented at $200 \mathrm{~g}$ for $5 \mathrm{~min}$ and seed in fresh culture medium.

To inhibit mitochondrial or cytosolic translation before harvesting, the medium was supplemented with $50 \mu \mathrm{g} / \mathrm{ml}$ chloramphenicol for $16 \mathrm{~h}$ or $100 \mu \mathrm{g} / \mathrm{ml}$ cycloheximide for $2 \mathrm{~h}$ respectively.

\subsection{Molecular biology methods}

\subsubsection{Determination of nucleic acid concentrations}

DNA and RNA concentrations were determined using the NanoVue spectrophotometer (GEHealthcare) assuming that $50 \mu \mathrm{g} / \mathrm{ml}$ DNA or $40 \mu \mathrm{g} / \mathrm{ml}$ RNA solutions would correspond to an $\mathrm{OD}_{260}$ of 1.

\subsubsection{DNA electrophoresis}

DNA fragments were separated in a gel of $1 \%$ agarose in TAE $(40 \mathrm{mM}$ Tris/acetate, $\mathrm{pH} 8.0$, $2 \mathrm{mM}$ EDTA) supplemented with $1 \mu \mathrm{g} / \mathrm{ml}$ ethidium bromide. Before loading, DNA samples were mixed with loading dye (4x stock: $40 \%$ saccharose, $1 \%$ OrangeG) and electrophoresis was performed in Mini-Sub Cell GT chambers (Biorad) for $30 \mathrm{~min}$ at $120 \mathrm{~V}$. As a standard, 
GeneRuler DNA Ladder Mix (Fermentas) was used. DNA bands were documented using an UV-transilluminator or cut out using a UV-light table.

\subsubsection{Purification of plasmids}

Plasmids were isolated from E. coli using the Wizard Plus SV Minipreps DNA Purification System (Promega) or NucleoBond PC 100 (Macherey-Nagel) according to the manufacturer's instructions.

\subsubsection{PCR-amplification of DNA fragments}

DNA fragments were amplified for cloning and for in vitro transcription by PCR using KOD Hot Start DNA Polymerase (Novagen) according to the recommendations of the manufacturer. Standard PCR reactions were performed in $50 \mu 1$ scale. Templates for amplification were either $25 \mathrm{ng}$ plasmid DNA or $200 \mathrm{ng}$ yeast genomic DNA (purified using the High Pure PCR Template Preparation Kit, Roche).

\subsubsection{Molecular cloning}

Using yeast genomic DNA or cDNA clones of human genes as a template, PCR was used to amplify inserts and introduce restriction sites for cloning. The PCR products were verified by agarose gel electrophoresis and purified using the Wizard SV Gel and PCR Clean-Up System (Promega).

Restriction of vector backbones and insert DNA-fragments was performed using Fast Digest restriction enzymes (Fermentas) and fragments were again purified. Subsequently the fragments were ligated using the Rapid DNA Ligation Kit (Fermentas) and the ligated constructs were transformed in E. coli. The clones were verified by restriction analysis and sequencing.

\subsubsection{Site-directed mutagenesis of plasmids}

The QuikChange Site-Directed Mutagenesis Kit (Agilent) was used to introduce point mutations in previously cloned genes. For this purpose complementary primers were designed which contained the nucleotide exchanges. Mutagenesis was performed according to the manufacturer's instructions and success was verified by sequencing. 


\subsubsection{Sequencing of DNA}

Sequencing of DNA was performed by the Sanger method using the BigDye Terminator v1.1 Cycle Sequencing Kit: The sequencing reaction was performed in $10 \mu$ scale containing $300 \mathrm{ng}$ plasmid, $8 \mathrm{pmol}$ primer, $1.5 \mu \mathrm{l}$ sequencing-mix and $1.5 \mu \mathrm{l}$ sequencing-buffer. After the sequencing reaction $\left(25\right.$ cycles: $\left.96{ }^{\circ} \mathrm{C}, 10 \mathrm{sec} ; 55^{\circ} \mathrm{C}, 15 \mathrm{sec} ; 60{ }^{\circ} \mathrm{C}, 4 \mathrm{~min}\right), 1 \mu 1125 \mathrm{mM}$ EDTA and $1 \mu 13 \mathrm{M}$ sodium acetate was added and DNA was precipitated by adding $50 \mu \mathrm{l}$ $100 \%$ ethanol. Subsequently the DNA was pelleted (20000 g, $15 \mathrm{~min}$ ), washed with $70 \%$ ethanol and dried (vacuum concentrator). After the DNA was resuspended in $15 \mu \mathrm{Hi}$-Diformamide it was analyzed using the Genetic Analyzer 3100 (Applied Biosystems) in the Göttingen Center for Molecular Biosciences.

\subsubsection{Transformation of $E$. coli}

Competent $E$. coli cells were prepared from a $100 \mathrm{ml}$ culture of $\mathrm{OD}_{600} \sim 0.6$. The culture was incubated at $4{ }^{\circ} \mathrm{C}$ for $5 \mathrm{~min}$ and harvested $\left(5000 \mathrm{~g}, 5 \mathrm{~min}, 4^{\circ} \mathrm{C}\right)$. Subsequently cells were resuspended in $40 \mathrm{ml}$ of ice cold $30 \mathrm{mM}$ potassium acetate, $100 \mathrm{mM}$ rubidium chloride, $10 \mathrm{mM}$ calcium chloride, $50 \mathrm{mM}$ manganese(II) chloride, 15\% glycerol, $\mathrm{pH} 5.8$ and incubated on ice for $5 \mathrm{~min}$. Cells were pelleted again, resuspended in $4 \mathrm{ml} 10 \mathrm{mM}$ rubidium chloride, $75 \mathrm{mM}$ calcium chloride, 15\% glycerin, $10 \mathrm{mM}$ MOPS, $\mathrm{pH}$ 6.8, and aliquots of $100 \mu 1$ were stored at $-80^{\circ} \mathrm{C}$.

For transformation, competent E. coli cells were thawed on ice and $25 \mathrm{mM}$ 2mercaptoethanol was added. After $10 \mathrm{~min}$ of incubation on ice, $25 \mathrm{ng}$ plasmid DNA or a ligation reaction was added to the cells. After $30 \mathrm{~min}$ incubation on ice and a heat shock at $42{ }^{\circ} \mathrm{C}$ for $45 \mathrm{sec}, 1 \mathrm{ml} \mathrm{LB}$ was added and cells were further incubated at $37^{\circ} \mathrm{C}$ for $60 \mathrm{~min}$. Cells were sedimented and plated on a LB plate supplemented with appropriate antibiotics for plasmid selection.

\subsubsection{Transformation of S. cerevisiae}

Yeast strains were transformed with plasmids and constructs for integration into the genome using the lithium acetate method, first described by Ito et al. (1983) and improved since then. A detailed protocol is described in Gietz and Schiestl (2007). For integration of constructs in the yeast genome, the integration-cassettes were amplified by PCR as described (Janke et al., 
2004). The integration in the genome was validated by PCR or by Western blot analysis of the target protein. The strains generated in this work are listed in table 2.4.

\subsection{Biochemical Methods}

\subsubsection{Determination of protein concentrations}

Protein concentration was determined by Bradford analysis using Roti-Quant (Roth) according to the manufacturer's recommendations. The absorbance of the protein solutions at $600 \mathrm{~nm}$ was measured in microtiter plates or cuvettes using a Spectra ELISA Reader (Tecan) or a BioPhotometer (Eppendorf), respectively. A standard curve was prepared using bovine IgG or BSA.

\subsubsection{Preparation of whole cell extracts}

Protein extracts from yeast cells were prepared as described (Yaffe and Schatz, 1984) with minor modifications: Cells were sedimented $\left(20000 \mathrm{~g}, 10 \mathrm{~min}, 4{ }^{\circ} \mathrm{C}\right)$ and resuspended in $\mathrm{H}_{2} \mathrm{O}$. After $250 \mathrm{mM}$ sodium hydroxide and $15 \mathrm{mM}$ 2-mercaptoethanol was added, cells were lyzed on ice for $10 \mathrm{~min}$. By addition of $6 \%$ TCA and incubation for $10 \mathrm{~min}$ on ice, protein was precipitated and could be sedimented by centrifugation $\left(20000 \mathrm{~g}, 2 \mathrm{~min}, 4^{\circ} \mathrm{C}\right)$.

\subsubsection{SDS-PAGE}

Denaturating protein electrophoresis (Laemmli, 1970) was performed using standard methods: Protein samples were resuspended in SDS-sample-buffer, incubated at $95{ }^{\circ} \mathrm{C}$ for $5 \mathrm{~min}$ and subsequently loaded on a polyacrylamide gel. In this study, gels were used, containing $0.1 \%$ SDS and 4\% acrylamide for the stacking gel or $12-14 \%$ acrylamide for the resolving gel. Acrylamide stock solutions contained 30\% acrylamide/bisacrylamide (37.5:1) with the exception for in vivo labeling experiments (see section 2.9) where a ratio of $75: 1$ acrylamide/bisacrylamide was used. Electrophoresis was performed using the MINI-Protean II (Biorad) system $(200 \mathrm{~V}, 25 \mathrm{~mA}$ per gel) or custom-made midi gel systems $(230 \mathrm{~V}, 30 \mathrm{~mA}$ per gel). As a marker the SDS-PAGE standard Broad Range (Biorad) was used.

SDS-sample-buffer: $10 \%$ glycerol, 2\% SDS, 0.01\% Bromphenol blue, 0.5\% 2-mercaptoethanol, $60 \mathrm{mM}$ Tris, pH 6.8

SDS-running-buffer: $25 \mathrm{mM}$ Tris, $191 \mathrm{mM}$ glycine, 0.1\% SDS 
Stacking-gel-buffer (10x): $0.8 \mathrm{M}$ Tris/HCl, pH 6.8

Resolving-gel-buffer (5x): $1.875 \mathrm{M}$ Tris/ $\mathrm{HCl}, \mathrm{pH} 8.8$

\subsubsection{BN-PAGE}

BN-PAGE (Schägger and von Jagow, 1991) was used to separate native protein complexes as described (Dekker et al., 1997): Mitochondria were resuspended in solubilization-buffer (1 $\mu 1$ buffer per $\mu \mathrm{g}$ protein) and incubated on ice for $30 \mathrm{~min}$. Unsoluble material was sedimented (20000 g, $15 \mathrm{~min}, 4{ }^{\circ} \mathrm{C}$ ) and the supernatant was supplemented with BN-sample-buffer to a final concentration of $1 \mathrm{x}$. After further incubation $\left(5 \mathrm{~min}, 4{ }^{\circ} \mathrm{C}\right)$ and a clarifying spin (20000 g, $5 \mathrm{~min}, 4{ }^{\circ} \mathrm{C}$ ) the sample was loaded on a $4-13 \%$ or $6-16 \%$ gradient gel with a $4 \%$ stacking gel. Electrophoresis was performed using the SE600 Ruby system (GE-Healthcare) at $100 \mathrm{~V}$ over night or $600 \mathrm{~V}$ for 4 - 5 hours. In case of subsequent Western blot analysis the cathode buffer was exchanged to buffer without Coomassie brilliant blue in the middle of the run. As a marker the HMW calibration kit (GE Healthcare) was used.

Solubilization-buffer: $1 \%$ digitonin, $20 \mathrm{mM}$ Tris/HCl, $\mathrm{pH}$ 7.4, $0.1 \mathrm{mM}$ EDTA, $50 \mathrm{mM}$ sodium chloride, 10\% glycerol, $1 \mathrm{mM}$ PMSF

BN-sample-buffer (10x): 5\% Coomassie brilliant blue G-250, $500 \mathrm{mM}$ e-amino n-caproic acid, $100 \mathrm{mM}$ BisTris, $\mathrm{pH} 7.0$

BN-gel buffer (3x): 200 mM $\varepsilon$-amino n-caproic acid. 150 mM Bis-Tris, $\mathrm{pH} 7.0$

BN-anode buffer: $50 \mathrm{mM}$ Bis-Tris/HCl, $\mathrm{pH} 7.0$

BN-cathode buffer: $50 \mathrm{mM}$ Tricine, $15 \mathrm{mM}$ Bis-Tris, $0.2 \%$ Coomassie brilliant blue G-250

Acrylamide stock solution: $48 \%$ acrylamide, $1.5 \%$ bisacrylamide (= 49.5\% T, 3\% C)

\subsubsection{D BN-PAGE/SDS-PAGE}

For 2 dimensional BN-PAGE/SDS-PAGE (Wittig et al., 2006) complexes were separated by BN-PAGE, lanes were cut and subjected to SDS-PAGE in the second dimension using same buffers and settings as described previously.

\subsubsection{Western blotting}

After proteins were separated by polyacrylamide-gelelectrophoresis, they were transferred to PVDF membranes, using semi dry blotting chambers (Peqlab). Gels, PVDF membranes, and Whatman papers were soaked in blotting buffer (20 mM Tris, $150 \mathrm{mM}$ glycine, $0.02 \%$ SDS, $20 \%$ methanol), assembled and finally blotting was performed at $250 \mathrm{~mA}$ for 2 hours. 


\subsubsection{Immunodetection of proteins on PVDF membranes}

PVDF membranes were blocked using 5\% milk powder in TBST (125 mM sodium chloride, $20 \mathrm{mM}$ Tris, $0.1 \%$ Tween $20, \mathrm{pH} 7.5$ ) for 1 hour at room temperature or at $4{ }^{\circ} \mathrm{C}$ over night. Subsequently, membranes were incubated in primary antibodies for 1 hour at room temperature and washed 3 times for 15 min with TBST. After incubation with HRP coupled secondary antibodies for 1 hour, membranes were washed again 3 times and signals were detected using the ECL system and X-Ray films (GE Healthcare).

\subsubsection{Coomassie staining}

Proteins on PVDF membranes or in polyacrylamide gels were Coomassie-stained using $2.5 \mathrm{~g} / 1$ Coomassie brilliant blue R-250, 40\% ethanol, 10\% acetic acid. After staining of PVDF membranes $(5 \mathrm{~min}$ ) or gels (3 hours) the background staining was removed by incubation in $40 \%$ ethanol, $10 \%$ acetic acid. Additionally PVDF membranes were completely destained using methanol.

\subsubsection{Colloidal Coomassie staining}

Highly sensitive protein staining in polyacrylamide gels was performed by Colloidal Coomassie staining (Neuhoff et al., 1988). Gels were fixed in 50\% methanol, $2 \%$ phosphoric acid for $16 \mathrm{~h}$ and washed three times with $\mathrm{H}_{2} \mathrm{O}$ for $30 \mathrm{~min}$ before they were incubated for $1 \mathrm{~h}$ in $34 \%$ methanol, $2 \%$ phosphoric acid, $17 \%(\mathrm{w} / \mathrm{v})$ ammonium sulfate. Subsequently gels were stained in $34 \%$ methanol, $2 \%$ phosphoric acid, 17\% (w/v) ammonium sulfate, $0.66 \mathrm{~g} / 1$ Coomassie brilliant blue G-250 for 3 days and destained with $\mathrm{H}_{2} \mathrm{O}$.

\subsubsection{Drying of polyacrylamide gels}

Polyacrylamide gels were placed on Whatman paper, covered by a plastic bag and subsequently dried using a vacuum gel drier (Scie-Plas) at $65^{\circ} \mathrm{C}$ for $2 \mathrm{~h}$.

\subsubsection{Autoradiography and quantification of radiolabeled proteins}

To detect radiolabeled proteins, separated by SDS-PAGE or BN-PAGE, gels were stained, dried and exposed to Storage Phosphor Screens (GE-Healthcare). After exposure for appropriate times, the signals were digitized using the Storm820 scanner (GE-Healthcare). 
Quantification of radiolabeled proteins was performed using ImageQuant TL software (GEHealthcare).

\subsection{Isolation of mitochondria}

\subsubsection{Isolation of yeast mitochondria}

Yeast mitochondria were isolated as described (Meisinger et al., 2006). In brief, yeast cultures were grown to $\mathrm{OD}_{600}$ of 2 and harvested by centrifugation at $7000 \mathrm{~g}$ for $15 \mathrm{~min}$. Cells were washed with $\mathrm{H}_{2} \mathrm{O}$ and incubated $30 \mathrm{~min}$ in $10 \mathrm{mM} \mathrm{DTT}, 100 \mathrm{mM}$ Tris $/ \mathrm{H}_{2} \mathrm{SO}_{4}$, $\mathrm{pH} 9.4(2 \mathrm{ml} / \mathrm{g}$ of cells $)$ at $30^{\circ} \mathrm{C}$. Subsequently cells were washed with zymolyase-buffer (1.2 $\mathrm{M}$ sorbitol, $20 \mathrm{mM}$ potassium phosphate, $\mathrm{pH}$ 7.4) and spheroplasted using Zymolyase$20 \mathrm{~T}\left(4 \mathrm{mg} / \mathrm{g}\right.$ of cells) in zymolyase-buffer $\left(7 \mathrm{ml} / \mathrm{g}\right.$ of cells) for $45 \mathrm{~min}$ at $30{ }^{\circ} \mathrm{C}$. Spheroplasts were washed in zymolyase-buffer, resuspended in ice cold homogenization buffer $(0.6 \mathrm{M}$ sorbitol, $10 \mathrm{mM}$ Tris/HCl, pH 7.4, 1 mM EDTA, $1 \mathrm{mM} \mathrm{PMSF,} \mathrm{0.2 \%} \mathrm{BSA)} \mathrm{and} \mathrm{homogenized}$ using a glass / teflon homogenizer (Potter S, Sartorius). From this extract, cell debris and the nuclear fraction was sedimented $\left(2000 \mathrm{~g}, 10 \mathrm{~min}, 4^{\circ} \mathrm{C}\right)$. Subsequently the supernatant was centrifuged $\left(17000 \mathrm{~g}, 15 \mathrm{~min}, 4^{\circ} \mathrm{C}\right)$ to pellet the mitochondrial fraction.

Mitochondria were washed with SEM (250 mM sucrose, $1 \mathrm{mM}$ EDTA, $10 \mathrm{mM}$ MOPS, $\mathrm{pH}$ 7.2), protein concentration was determined by Bradford analysis using bovine IgG as a standard and mitochondria were diluted to $10 \mathrm{mg} / \mathrm{ml}$ with SEM. After shock freezing in liquid nitrogen mitochondria were stored at $-80{ }^{\circ} \mathrm{C}$.

It should also be noted that incubation steps were performed at the respective temperatures when mitochondria were isolated from cultures that were grown at nonpermissive temperatures (e.g. $\left.19^{\circ} \mathrm{C}\right)$.

\subsubsection{Isolation of mitochondria from human cells}

Mitochondria of human cultured cells (HEK 293T) were isolated as described (Lazarou et al., 2009). Cells were harvested, washed with PBS (200 g, 5 min) and homogenized in $220 \mathrm{mM}$ mannitol, $70 \mathrm{mM}$ sucrose, $20 \mathrm{mM}$ Hepes, pH 7.6, $1 \mathrm{mM}$ EDTA, $0.5 \mathrm{mM}$ PMSF using a glass / teflon homogenizer (Potter S, Sartorius). Cell debris and nuclei were removed by centrifugation $\left(800 \mathrm{~g}, 10 \mathrm{~min}, 4^{\circ} \mathrm{C}\right)$ and mitochondria were pelleted at $10000 \mathrm{~g}, 10 \mathrm{~min}$, $4{ }^{\circ} \mathrm{C}$. Protein concentration was determined by Bradford analysis using BSA as standard. 
Table 2.8 Conditions for in vitro translation of precursor proteins

\begin{tabular}{ll}
\hline Precursor & In vitro translation-system (comments) \\
\hline Shy1 $^{\text {a }}$ & TNT SP6 Quick Coupled Transcription/Translation System \\
OTC & TNT SP6 Quick Coupled Transcription/Translation System \\
SURF1 & Flexi Rabbit Reticulocyte Lysate System (100 mM KCl extra) \\
COX6A1 & TNT SP6 Quick Coupled Transcription/Translation System \\
Uth1 & Flexi Rabbit Reticulocyte Lysate System (120 mM KCl and 2 mM MgOAc extra) \\
Sym1 ${ }^{\text {a }}$ & TNT SP6 Quick Coupled Transcription/Translation System \\
AAC & TNT SP6 Quick Coupled Transcription/Translation System \\
Sym1-truncations & Flexi Rabbit Reticulocyte Lysate System (70 mM KCl extra) \\
\hline
\end{tabular}

awild-type and mutant forms

\subsection{In vitro import analysis in isolated mitochondria}

\subsubsection{In vitro labeling of precursor proteins}

\subsubsection{In vitro transcription}

RNA was transcribed from DNA templates using the SP6 RNA polymerase. Therefore PCR products were used, amplified from yeast genomic DNA (the SP6 promoter was introduced with the primers), or the open reading frame was first cloned under the control of the SP6 promoter in pGEM4Z and the plasmid was used as a template for PCR (using an SP6 and T7 primers). After purification of the PCR-products, in vitro transcription was performed using the mMESSAGE mMACHINE SP6 Kit (Ambion) and the RNA was purified using the MEGAclear kit (Ambion) according to the manufacturer's recommendations.

\subsubsection{In vitro translation}

Proteins were labeled with $\left[{ }^{35} \mathrm{~S}\right]$ methionine by in vitro translation from purified RNA using the Flexi Rabbit Reticulocyte Lysate System (Promega): $33 \mu 1$ of Lysate, $1 \mu 1$ of amino acid mix, $50 \mu \mathrm{Ci}$ of $\left[{ }^{35} \mathrm{~S}\right]$ methionine, $1 \mu \mathrm{g}$ RNA was supplemented with different concentrations of potassium chloride and magnesium acetate (see table 2.8), and adjusted to a total volume of $50 \mu \mathrm{l}$ with $\mathrm{H}_{2} \mathrm{O}$. After in vitro translation for $90 \mathrm{~min}$ at $30^{\circ} \mathrm{C}$, the reaction was stopped on 
ice and excess methionine $(20 \mathrm{mM})$ and $250 \mathrm{mM}$ sucrose was added. Lysates were frozen in liquid nitrogen and stored at $-80^{\circ} \mathrm{C}$.

\subsubsection{Coupled in vitro transcription/translation}

Coupled transcription/translation of radiolabeled proteins was performed with the TNT SP6 Quick Coupled Transcription/Translation System (Promega): Open reading frames were cloned under control of the SP6 promoter in pGEM4Z and plasmids were purified. $50 \mu 1$ lysate mix was supplemented with $1 \mu \mathrm{g}$ plasmid DNA and $50 \mu \mathrm{Ci}\left[{ }^{35} \mathrm{~S}\right]$ methionine and incubated at $30{ }^{\circ} \mathrm{C}$ for $90 \mathrm{~min}$. The reaction was stopped on ice and $20 \mathrm{mM}$ methionine and $250 \mathrm{mM}$ sucrose was added. The lysate was frozen in liquid nitrogen and stored at $-80{ }^{\circ} \mathrm{C}$.

\subsubsection{In vitro import into isolated mitochondria}

Radiolabeled precursor proteins were in vitro imported into isolated yeast or human mitochondria as previously described in Wiedemann et al. (2006b) or Lazarou et al. (2009) respectively.

First, mitochondria were resuspended in Import-buffer to $0.5-0.75 \mathrm{mg} / \mathrm{ml}$. As a negative control the membrane potential was dissipated by addition of $1 \%$ AVO mix $(1 \mathrm{mM}$ antimycin A, $0.1 \mathrm{mM}$ valinomycin, $2 \mathrm{mM}$ oligomycin in ethanol). After addition of $5-10 \%$ of the radiolabeled precursor protein lysate, import was performed at $25^{\circ} \mathrm{C}$ (yeast) or $37^{\circ} \mathrm{C}$ (human) before it was stopped on ice and by addition of $1 \%$ AVO mix. If indicated the unimported precursor protein was degraded by addition of $40 \mu \mathrm{g} / \mathrm{ml}$ proteinase $\mathrm{K}$, which was inactivated with $2 \mathrm{mM}$ PMSF after incubation for $10 \mathrm{~min}$ at $4{ }^{\circ} \mathrm{C}$.

After import, mitochondria were reisolated $\left(20000 \mathrm{~g}, 10 \mathrm{~min}, 4^{\circ} \mathrm{C}\right)$, washed with SEM and subsequently analyzed by SDS-PAGE or BN-PAGE.

Import analysis in temperature sensitive, mutant yeast mitochondria was performed at $25^{\circ} \mathrm{C}$, as described, with an additional heat shock $\left(15 \mathrm{~min}, 37^{\circ} \mathrm{C}\right)$ after resuspension in Import-buffer.

For antibody shift experiments after in vitro import, mitochondria were resuspended in SEM to $1 \mathrm{mg} / \mathrm{ml}$ and osmotic swelling of the mitochondrial outer membrane was performed by addition of 10 volumes of $1 \mathrm{mM}$ EDTA, $10 \mathrm{mM} \mathrm{MOPS} / \mathrm{KOH}, \mathrm{pH}$ 7.2. After addition of antibody (1\% serum) samples were incubated for $45 \mathrm{~min}$ on ice, mitochondria were washed with SEM and samples were analyzed by BN-PAGE analysis.

Import-buffer (human): $250 \mathrm{mM}$ sucrose, $5 \mathrm{mM}$ magnesium acetate, $80 \mathrm{mM}$ potassium acetate, $20 \mathrm{mM}$ Hepes /KOH, pH 7.4, 2 mM ATP, $2 \mathrm{mM} \mathrm{NADH,} 10 \mathrm{mM}$ sodium succinate, $10 \mathrm{mM}$ malic acid 
Import-buffer (yeast): $3 \% \mathrm{BSA}, 250 \mathrm{mM}$ sucrose, $80 \mathrm{mM}$ potassium chloride, $5 \mathrm{mM}$ magnesium chloride, $2 \mathrm{mM}$ potassium phosphate, $5 \mathrm{mM}$ methionine, $10 \mathrm{mM} \mathrm{MOPS} / \mathrm{KOH}, \mathrm{pH} 7.2,2 \mathrm{mM}$ ATP, $2 \mathrm{mM} \mathrm{NADH}$ (for assembly studies $5 \mathrm{mM}$ creatin phosphate and $100 \mu \mathrm{g} / \mathrm{ml}$ Creatin Kinase was added as ATP-regenerating system)

\subsubsection{Stability assay after in vitro import}

To analyze the stability of proteins after import a pulse/chase study was performed (Röttgers et al., 2002). In vitro import was performed for $5 \mathrm{~min}$ in $500 \mu 1$ reactions using $375 \mu \mathrm{g}$ isolated yeast or human mitochondria at $25^{\circ} \mathrm{C}$ or $37^{\circ} \mathrm{C}$, respectively. The import was stopped by addition of $1 \%$ AVO mix and samples of $50 \mu 1$ were taken before and after the precursor protein was degraded by incubation with $40 \mu \mathrm{g} / \mathrm{ml}$ proteinase $\mathrm{K}$ for $10 \mathrm{~min}$ on ice. Mitochondria were reisolated $\left(20000 \mathrm{~g}, 10 \mathrm{~min} 4{ }^{\circ} \mathrm{C}\right)$ and resuspended in pre-warmed, energized Import-buffer. Chase samples of $50 \mu 1$ were taken after incubation at $25^{\circ} \mathrm{C}$ (yeast) or $37^{\circ} \mathrm{C}$ (human) for the indicated times. All samples were analyzed by SDS-PAGE and digital autoradiography.

\subsection{In vivo labeling of mitochondrial translation products}

Translation efficiency of mitochondria-encoded proteins in different yeast strains was analyzed as previously described (Mick et al., 2010). Yeast cultures in SGal-ura or SGal were grown to $\mathrm{OD}_{600}$ of 1.0 and $1.0 \mathrm{OD}_{600}$ equivalent of cells was harvested by centrifugation (20000 g, $2 \mathrm{~min}$ ). Cells were washed and resuspended in $1 \mathrm{ml}$ Labeling-buffer ( $2 \%$ galactose, $40 \mathrm{mM}$ potassium phosphate, $\mathrm{pH}$ 6.0). After incubation at the respective labeling temperature $\left(30^{\circ} \mathrm{C}\right.$ or $19^{\circ} \mathrm{C}$ ) for $10 \mathrm{~min}$, cytosolic translation was stopped by addition of cycloheximide to $150 \mu \mathrm{g} / \mathrm{ml}$. Labeling was started after $5 \mathrm{~min}$ incubation by addition of $40 \mu \mathrm{Ci}$ $\left[{ }^{35} \mathrm{~S}\right]$ methionine. After 5 or $15 \mathrm{~min}$ labeling at $30^{\circ} \mathrm{C}\left(10\right.$ or $30 \mathrm{~min}$ at $\left.19{ }^{\circ} \mathrm{C}\right)$, samples were taken, excess methionine $(4 \mathrm{mM})$ was added, and after further 2 min incubation put on ice. Whole cell extracts of these samples were analyzed by SDS-PAGE and digital autoradiography.

Stability of mitochondria-encoded proteins was analyzed by in vivo labeling (pulse) followed by further incubation (chase) after mitochondrial translation has been stopped: Mitochondrial translation products of $1.0 \mathrm{OD}_{600}$ equivalent of cells were labeled as described for $15 \mathrm{~min}$ at $30{ }^{\circ} \mathrm{C}$. Labeling was stopped by addition of $4 \mathrm{mM}$ methionine and $100 \mu \mathrm{g} / \mathrm{ml}$ chloramphenicol and cells were further incubated at $30^{\circ} \mathrm{C}$. After 2 min a pulse sample was 
taken. Chase samples were taken after additional incubation for 5, 15, 45 or $135 \mathrm{~min}$. Samples were analyzed by SDS-PAGE of whole cell extracts and digital autoradiography.

\subsection{Localization and topology of mitochondrial proteins}

\subsubsection{Subcellular localization analysis by fluorescence microscopy}

Proteins, containing a GFP tag, were localized by in vivo fluorescence microscopy. Yeast cultures were grown in SG medium to mid $\log$ phase at $30^{\circ} \mathrm{C}$. Mitochondria were stained by addition of $0.2 \mu \mathrm{g} / \mathrm{ml}$ MitoTracker Orange CMTMRos (Invitrogen) to the culture for $15 \mathrm{~min}$. Subsequently $5 \mu$ of the culture was transferred to a glass slide, a cover slip was fixed and microscopy was performed using a DeltaVision Deconvolution microscope (Olympus IX71, Applied Precision). Fluorescence of GFP or MitoTracker was detected using FITC or TRITC filter setup, respectively. Whole cells were visualized using differential interference contrast optics.

\subsubsection{Submitochondrial localization analyses}

Submitochondrial localization of proteins was determined by protease protection assays as described (Mick et al., 2007) in intact mitochondria and mitoplasts, generated by osmotic swelling of the outer membrane with $1 \mathrm{mM}$ EDTA, $10 \mathrm{mM} \mathrm{MOPS/KOH,} \mathrm{pH} \mathrm{7.2.}$ Mitochondria or mitoplasts $(1 \mathrm{mg} / \mathrm{ml})$ were incubated with proteinase $\mathrm{K}(0-90 \mu \mathrm{g} / \mathrm{ml})$ for $10 \mathrm{~min}$ on ice. Proteinase $\mathrm{K}$ was inactivated by addition of $2 \mathrm{mM}$ PMSF and samples were analyzed by SDS-PAGE and Western blotting.

\subsubsection{Analysis of membrane association by carbonate treatment}

To distinguish peripheral membrane association from membrane integration of proteins, carbonate extraction was performed as described (Mick et al., 2007). Incubation of isolated mitochondria $(0.5 \mathrm{mg} / \mathrm{ml})$ in $100 \mathrm{mM}$ sodium carbonate $(\mathrm{pH} 11.5)$ for $20 \mathrm{~min}$ on ice releases peripheral membrane proteins. Subsequently, membranes and transmembrane proteins were sedimented by ultracentrifugation $\left(100000 \mathrm{~g}, 45 \mathrm{~min}, 4^{\circ} \mathrm{C}\right)$. 


\subsection{Purification of mitochondrial protein complexes}

\subsubsection{IgG-chromatography}

Mitochondrial complexes of ProteinA tagged proteins were isolated as described (Geissler et al., 2002; Rehling et al., 2003). Isolated yeast mitochondria were resuspended to $1 \mathrm{mg} / \mathrm{ml}$ in Solubilization-buffer, unsoluble material was spun out $\left(20000 \mathrm{~g}, 15 \mathrm{~min}, 4{ }^{\circ} \mathrm{C}\right)$ and the supernatant was applied to IgG-sepharose $\left(150 \mu \mathrm{l}\right.$ beads). After binding for 3 hours at $4{ }^{\circ} \mathrm{C}$ and extensive washing using 20 column volumes Wash-buffer, the complexes were eluted by TEV-protease (Invitrogen) cleavage of the ProteinA-tag $\left(16 \mathrm{~h}, 6^{\circ} \mathrm{C}\right)$. Subsequently, the Histagged TEV-protease was removed by addition of $5 \mu 1$ Ni-NTA-Agarose (Qiagen) and the eluated fraction was analyzed by BN-PAGE analysis.

Solubilisation-buffer: $1 \%$ digitonin, $20 \mathrm{mM}$ Tris/HCl, $\mathrm{pH}$ 7.4, $0.1 \mathrm{mM}$ EDTA, $80 \mathrm{mM}$ sodium chloride, $10 \%$ glycerol, $1 \mathrm{mM}$ PMSF

Wash-buffer: $0.3 \%$ digitonin, $20 \mathrm{mM}$ Tris/ $\mathrm{HCl}, \mathrm{pH} 7.4,0.1 \mathrm{mM}$ EDTA, $80 \mathrm{mM}$ sodium chloride, $10 \%$ glycerol, $1 \mathrm{mM}$ PMSF

\subsubsection{Co-immunoprecipitation}

Isolated mitochondria were incubated $30 \mathrm{~min}$ at $4{ }^{\circ} \mathrm{C}$ in solubilization buffer $(1 \%$ digitonin, $60 \mathrm{mM}$ sodium chloride, $5 \mathrm{mM}$ EDTA, $10 \%$ glycerol, $20 \mathrm{mM}$ Tris/HCl, pH 7.4) and unsoluble material was spun out. The supernatant (total) was applied to antibodies, which were crosslinked (using dimethyl-pimelimidate) to ProteinA-Sepharose beads (GEHealthcare). After binding ( $60 \mathrm{~min}, 4{ }^{\circ} \mathrm{C}$ ) and extensive washing of the beads, bound proteins were eluted using $0.1 \mathrm{M}$ glycine $\mathrm{pH}$ 2.5. Subsequently samples were neutralized with $1 \mathrm{M}$ Tris and analyzed by SDS-PAGE and Western blotting.

\subsection{Electrophysiological characterization of mitochondrial proteins}

\subsubsection{Isolation of proteins from yeast mitochondria for electrophysiological characterization}

Mitochondrial proteins, fused to a ProteinA-His ${ }_{7}$ tag at their C-terminus, were purified by $\mathrm{Ni}^{2+}$ affinity- and subsequent IgG-chromatography followed by TEV-protease cleavage of the tag. $15-20 \mathrm{mg}$ isolated yeast mitochondria were centrifuged down $\left(20000 \mathrm{~g}, 10 \mathrm{~min}, 4^{\circ} \mathrm{C}\right)$, 
resuspended to $10 \mathrm{mg} / \mathrm{ml}$ in SDS-buffer and incubated $15 \mathrm{~min}$ at $25^{\circ} \mathrm{C}$. Subsequent $20 \mathrm{x}$ dilution with $0.2 \%$-triton-buffer $\left(0.5 \mathrm{mg} / \mathrm{ml}\right.$ protein) and incubation at $4{ }^{\circ} \mathrm{C}$ for $30 \mathrm{~min}$ allowed refolding of the proteins. After a clarifying spin $\left(20000 \mathrm{~g}, 15 \mathrm{~min}, 4{ }^{\circ} \mathrm{C}\right)$ the supernatant was subjected to $500 \mu \mathrm{l}$ Ni-NTA beads (Qiagen). After binding and extensive washing with 25 column volumes $0.1 \%$-triton-buffer, the bound proteins were eluted with $7.5 \mathrm{ml} 300 \mathrm{mM}$ imidazole in $0.1 \%$-triton-buffer. After dilution of the sample with $22.5 \mathrm{ml}$ $0.1 \%$-triton-buffer, $100 \mu \mathrm{IgG}$-sepharose beads were added and protein was bound at $4{ }^{\circ} \mathrm{C}$ for $16 \mathrm{~h}$. After extensive washing of the beads ( 25 column volumes with $0.1 \%$ triton buffer), bound protein was eluted by cleavage of the ProteinA tag (30 U TEV-protease, Invitrogen). TEV-protease was removed by addition of $10 \mu 1$ Ni-NTA beads and samples were subjected to electrophysiological analysis.

SDS-buffer: $1 \%$ SDS, $50 \mathrm{mM}$ sodium phosphate, $\mathrm{pH}$ 7.4, $100 \mathrm{mM}$ sodium chloride, 10\% glycerol, $10 \mathrm{mM}$ imidazole, $1 \mathrm{mM}$ PMSF, proteinase inhibitor cocktail (Complete EDTA free, Roche)

0.2\%-triton-buffer: $0.2 \%$ triton $\mathrm{X}-100,50 \mathrm{mM}$ sodium phosphate, $\mathrm{pH} 7.4,100 \mathrm{mM}$ sodium chloride, $10 \%$ glycerol, 10 mM imidazole, $1 \mathrm{mM}$ PMSF, proteinase inhibitor cocktail (Complete EDTA free, Roche)

0.1\%-triton-buffer: $0.1 \%$ triton $\mathrm{X}-100,50 \mathrm{mM}$ sodium phosphate, $\mathrm{pH} 7.4,100 \mathrm{mM}$ sodium chloride, $10 \%$ glycerol, $10 \mathrm{mM}$ imidazole, $1 \mathrm{mM}$ PMSF, proteinase inhibitor cocktail (Complete EDTA free, Roche)

\subsubsection{Electrophysiology}

Electrophysiological measurements were performed by Dr. M. Meinecke and V. Krüger (AG-Wagner, Universität Osnabrück).

Purified protein was refolded into liposomes using detergent mediated reconstitution. Proteoliposomes were fused to a planar lipid bilayer. Subsequently current recordings were performed at different holding potentials. For all measurements symmetrical buffer conditions were used (250 mM potassium chloride, $20 \mathrm{mM}$ MOPS/Tris, $\mathrm{pH}$ 7.0), except for the determination of the reverse potential, where $20 \mathrm{mM}$ potassium chloride, $20 \mathrm{mM}$ MOPS/Tris, pH 7.0 was used at the trans-side of the membrane and $250 \mathrm{mM}$ potassium chloride, $20 \mathrm{mM}$ MOPS/Tris, $\mathrm{pH} 7.0$ was used at the cis-side.

To validate the specificity of the pore forming activity, antibodies were added to each side of the lipid bilayer during current recordings. 


\section{Results}

\subsection{SHY1 as a model for SURF1 mutations, identified in Leigh Syndrome patients}

\subsubsection{SHY1 mutations, mimicking patient SURF1 alleles lead to cytochrome $c$ oxidase deficiency}

\subsubsection{Yeast models for SURF1 mutations show respiratory deficiency}

SURF1 is a highly conserved assembly factor of the human cytochrome $c$ oxidase (Zhu et al., 1998). Moreover, mutations in SURF1 are the most prevalent cause of Leigh Syndrome with isolated cytochrome $c$ oxidase deficiency (Pequignot et al., 2001): In patients, point mutations were identified, leading to an exchange of glycine ${ }^{124}$ of SURF1 to glutamic acid or arginine (Coenen et al., 1999; Poyau et al., 2000). Furthermore, Teraoka et al. (1999) reported an exchange of tyrosine ${ }^{274}$ of SURF1 to aspartic acid. Interestingly, these amino acids are highly conserved (Fig 3.1A), indicating their functional importance. In this study, these point mutations in SURF1 were analyzed, using the yeast homolog SHY1 as a model: The affected $\mathrm{G}^{124}$ or $\mathrm{Y}^{274}$ residues in SURF1 correspond to Shy $1 \mathrm{G}^{137}$ or $\mathrm{Y}^{344}$, respectively.

To map the site of these amino acid exchanges in Shy1, a prediction of transmembrane spans (Fig 3.1B) was compared to the topology of Shy1 (Mashkevich et al., 1997). This indicated that $\mathrm{G}^{137}$ of Shy1 resides in the IMS domain, whereas $\mathrm{Y}^{344}$ locates at the interface of the IMS domain and the second transmembrane span of Shy1 (Fig 3.1C).

To utilize $S H Y 1$ as a model for the SURF1 patient mutations, the $S H Y 1$ open reading frame with its endogenous promoter and terminator sequences was cloned into the yeast vector pRS416 and the point mutations were introduced using site directed mutagenesis. These plasmids, or an empty plasmid as control, were transformed in a shyls strain and growth analyses on fermentable and nonfermentable carbon sources were performed to test for respiratory competence (Fig 3.1D). All strains showed growth on fermentable carbon sources. Interestingly, the strain expressing Shy $1^{\mathrm{G} 137 \mathrm{E}}$ shows respiratory deficiency at all temperatures tested and resembles the shyl $\Delta$ phenotype as reported previously (Bestwick et al., 2010a). In contrast to that, a $\mathrm{G}^{137} \mathrm{R}$ exchange in Shy1 leads to a temperature dependent 
A

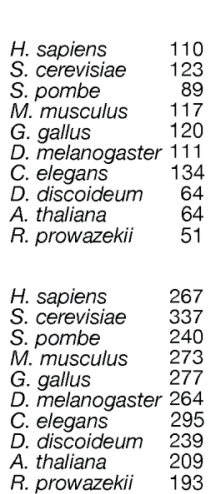

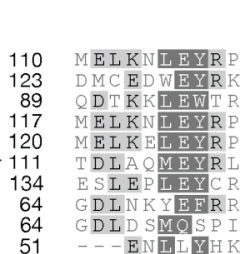
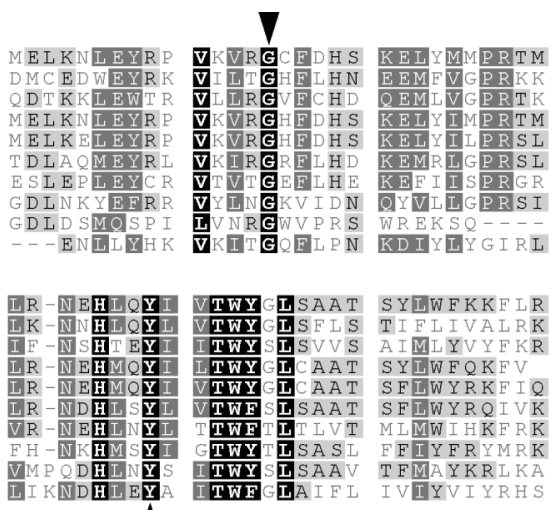

$\boldsymbol{\Lambda}$
B

139
152
118
146
149
140
163
93
89
77

295
365
268
300
305
296
323
267
238
222

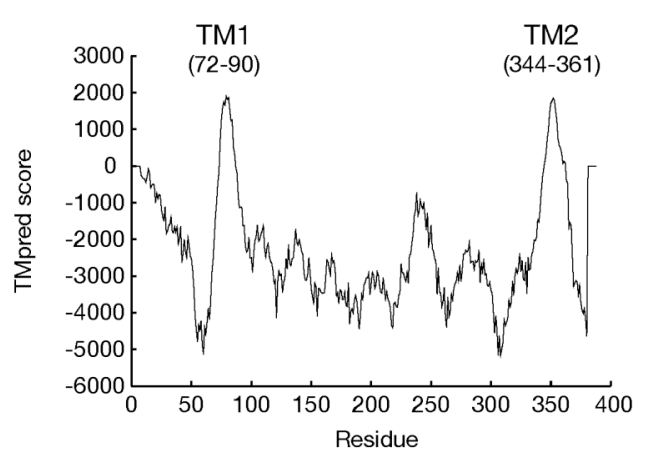

C

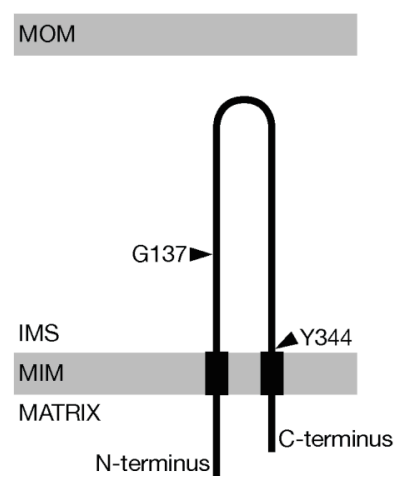

D

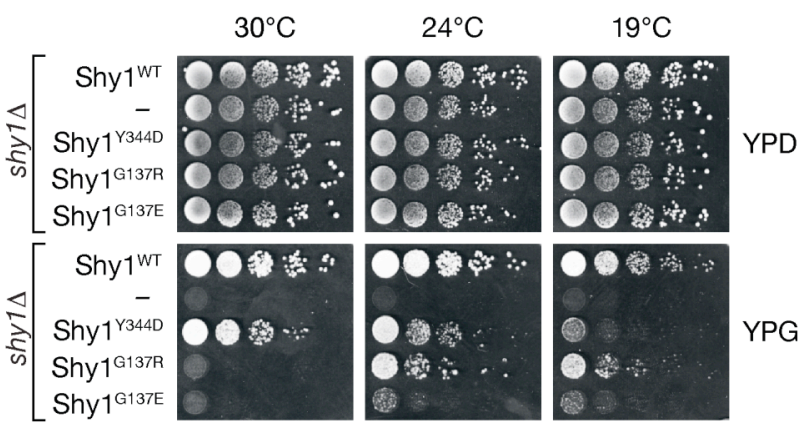

Figure 3.1 Mimicking highly conserved $S U R F 1$ patient mutations in yeast leads to respiratory deficiency

(A) Partial alignment of SURF1/Shy1 homolog proteins using ClustalW2 (Blosum62 score matrix) surrounding the amino acids $\mathrm{G}^{124}$ and $\mathrm{Y}^{274}$ of SURF1 (arrowheads), which are affected by pathogenic SURF1 mutations. Black boxes indicate $100 \%$, dark gray $80-100 \%$, light gray $60-80 \%$ similarity. (B) Prediction of transmembrane spans (TM) of Shyl, using TMpred. (C) Topology of Shyl, indicating the localization of amino acids $\mathrm{G}^{137}$ and $\mathrm{Y}^{344}$, corresponding to the affected SURF1 residues in Leigh Syndrome patients. MOM, mitochondrial outer membrane; IMS, inter membrane space; MIM, mitochondrial inner membrane. (D) Serial dilutions of shyl $\Delta$ cells, containing an empty plasmid (-) or expressing Shy ${ }^{\mathrm{WT}}$, Shy $1^{\mathrm{Y} 344 \mathrm{D}}$, Shy $1^{\mathrm{G} 137 \mathrm{R}}$ or Shy $1^{\mathrm{G} 137 \mathrm{E}}$ were spotted on fermentable (YPD) or nonfermentable (YPG) medium and were incubated at indicated temperatures.

respiratory deficiency. Cells expressing Shy $1^{\mathrm{G} 137 \mathrm{R}}$ do not grow at $30{ }^{\circ} \mathrm{C}$ but show residual growth at reduced temperatures. Remarkably, the strain expressing the Shy $1^{\mathrm{Y} 344 \mathrm{D}}$ variant however, showed a cold sensitive respiratory deficiency and only slightly reduced growth on non fermentable carbon sources at $30^{\circ} \mathrm{C}$.

\subsubsection{SHY1 mutant strains lack cytochrome $c$ oxidase}

To analyze the respiratory deficiency of yeast strains expressing the mutant variants of Shy 1 in more detail, mitochondria were isolated from these strains, grown at $30{ }^{\circ} \mathrm{C}$. Subsequently 
A

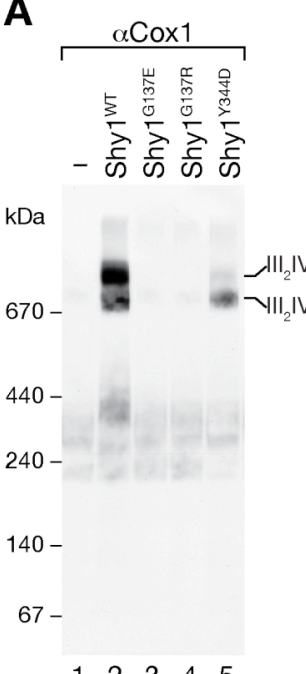

$\begin{array}{llllllll}1 & 2 & 3 & 4 & 5\end{array}$
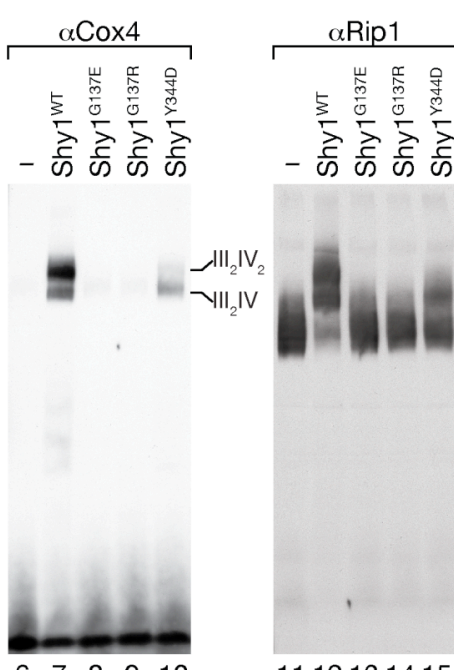

$\begin{array}{lllll}6 & 7 & 8 & 9 & 10\end{array}$
一旁高旁言
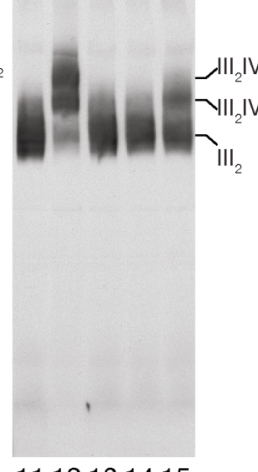

1112131415

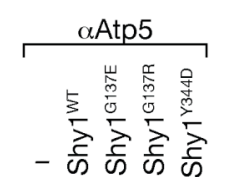

B

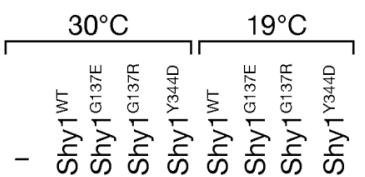

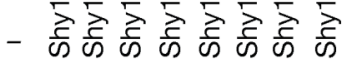

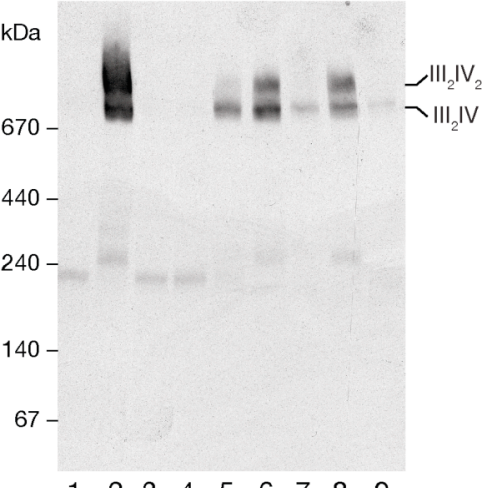

Figure 3.2 Point mutations in $S H Y 1$ lead to lack of cytochrome $c$ oxidase

(A) Mitochondria, isolated from indicated strains, grown at $30^{\circ} \mathrm{C}$ were solubilized and respiratory chain complexes were analyzed by BN-PAGE and Western blotting using indicated antibodies (B) Cytochrome $c$ oxidase of mitochondria, isolated from strains grown at $30^{\circ} \mathrm{C}$ or $19{ }^{\circ} \mathrm{C}$ was analyzed as in (A) using Cox1 specific antibody.

the respiratory chain complexes were analyzed by BN-PAGE and Western blotting (Fig 3.2A). Cytochrome $c$ oxidase was analyzed, using antibodies against the mitochondriaencoded core subunit Cox 1 and the nuclear-encoded subunit Cox4. As reported previously (Mashkevich et al., 1997; Nijtmans et al., 2001), in mitochondria from shyl $\Delta$ cells, no mature cytochrome $c$ oxidase could be detected. In contrast, cytochrome $c$ oxidase assembles to supercomplexes $\left(\mathrm{III}_{2} \mathrm{IV}, \mathrm{III}_{2} \mathrm{IV}_{2}\right)$ in wild-type mitochondria (Fig 3.2A lanes 2, 7). Explaining the growth phenotype, in mitochondria from shyl $1^{G 137 E}$ and $s h y 1^{G 137 R}$ cells, no mature cytochrome $c$ oxidase could be detected (Fig 3.2A lanes 3,8 and 4,9, respectively) and in shy $1^{Y 344 D}$ mitochondria, cytochrome $c$ oxidase could only be detected in minute amounts. Using Rip1 specific antibodies, the cytochrome $b c_{1}$ complex was analyzed. This complex was mainly present in its dimeric form $\left(\mathrm{III}_{2}\right)$ in the mutant mitochondria, and failed to form supercomplexes $\left(\mathrm{III}_{2} \mathrm{IV}_{1}\right.$ and $\left.\mathrm{III}_{2} \mathrm{IV}_{2}\right)$ due to the lack of cytochrome $c$ oxidase. As a control, the $\mathrm{F}_{1} \mathrm{~F}_{\mathrm{O}}$-ATPase complexes $\left(\mathrm{V}_{1}, \mathrm{~V}_{2}\right)$ were analyzed, which were unaffected by the SHY1 mutations.

To study the temperature sensitive respiratory phenotype, caused by the mutant Shy1 variants in more detail, mitochondria from cells grown at $19^{\circ} \mathrm{C}$ were analyzed by BN-PAGE and Western blotting (Fig 3.2B). In consistence with the growth phenotype at $19{ }^{\circ} \mathrm{C}$, cytochrome 
$c$ oxidase levels in shyl $1^{G 137 R}$ mitochondria were comparable to wild-type but were hardly detectable in mitochondria from the cold sensitive shyl ${ }^{Y 344 D}$ strain.

In all tested mutant strains, a lack of cytochrome $c$ oxidase could be shown, which was even enhanced at non-permissive temperatures. Therefore it can be concluded that the respiratory deficiency, observed in the mutant strains, is caused by the lack of cytochrome $c$ oxidase.

\subsubsection{Mutations affecting $\mathbf{G}^{137}$ of Shy1 lead to reduced abundance of Shy1}

It was reported that $S U R F 1$ mutations lead to reduction or absence of SURF1 in patient mitochondria (Pequignot et al., 2001). Furthermore, yeast Shyl is known to play a central role in Cox1 biogenesis as well as in early steps of cytochrome $c$ oxidase assembly (Barrientos et al., 2002; Mick et al., 2007). Therefore, steady state levels of Shyl as well as subunits and assembly factors of the cytochrome $c$ oxidase were analyzed in detail in mitochondria isolated from the SHY1 mutant strains (Fig 3.3). Shy1 was detected using antibodies raised against full length Shy1 or the C-terminus of Shy1, which is not affected by the mutations. Interestingly Shyl could hardly be detected with both antibodies in shyl ${ }^{\text {G137E }}$ and shyl $1^{G 137 R}$ mitochondria. Furthermore, Cox1 and Cox2, mitochondria-encoded core subunits of the cytochrome $c$ oxidase, were strongly reduced in $s h y 1^{G 137 E}$ and $s h y 1^{G 137 R}$ mitochondria, similar to mitochondria lacking Shy1. This findings are consistent with recent studies by Bestwick et al. (2010a). In contrast, Shy1 was present in comparable amounts in shy $1^{Y 344 D}$ and wild-type mitochondria. However, Cox1 and Cox2 also showed reduced
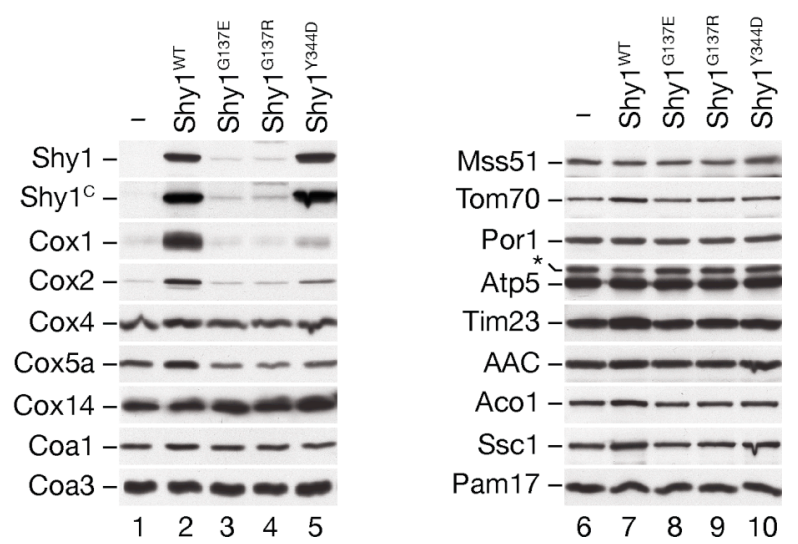

Figure 3.3 SHY1 point mutations lead to reduced abundance of Shy1 and core cytochrome $c$ oxidase subunits

$15 \mu \mathrm{g}$ mitochondria, isolated from indicated strains, were analyzed by SDS-PAGE and Western blotting. Asterisk, cross reactive band; Shy1, antibody against full-length Shy1; Shy ${ }^{\mathrm{C}}$, antibody against Cterminal peptide of Shy1. 
abundance in shyl $1^{Y 344 D}$ mitochondria. Moreover, nuclear-encoded subunits (Cox4, Cox5a) as well as early assembly factors (Cox14, Coa1, Coa3, Mss51) of the cytochrome $c$ oxidase were not affected by the SHY1 mutations. As controls, marker proteins of different mitochondrial sub-compartments were analyzed, which showed no differences in the mitochondria tested.

It can be concluded that the lack of Shy1 in mitochondria from shy $1^{G 137 E}$ and shy $1^{G 137 R}$ cells, explains their respiratory deficiency, which phenotypically resembles a deletion of SHY1.

\subsubsection{Import and stability of Shy1 in yeast mitochondria}

\subsubsection{Mutant Shy1 is efficiently imported into yeast mitochondria}

The observed absence of Shyl in shyl $1^{G 137 E}$ and $s h y l^{G 137 R}$ mitochondria could be caused by impaired mitochondrial import or by destabilization and rapid turnover of the mutant Shy 1 proteins.

To analyze mitochondrial import, wild-type and mutant Shy1 precursor proteins were synthesized and $\left[{ }^{35} \mathrm{~S}\right]$ labeled using rabbit reticulocyte lysate and an in vitro import assay was established as described in Mick et al. (2007) (Fig 3.4A and B). Upon import, the Shy1 precursor was processed to the faster migrating mature Shyl protein. Dependent on the membrane potential $(\Delta \psi)$, mature $\left[{ }^{35}\right.$ S $]$ Shy 1 accumulated in mitochondria and was protected

A

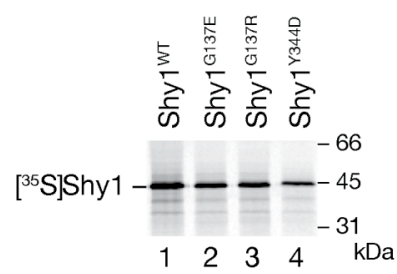

B

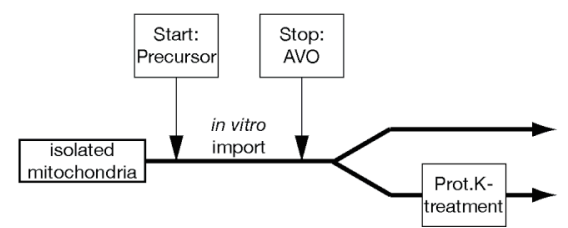

C

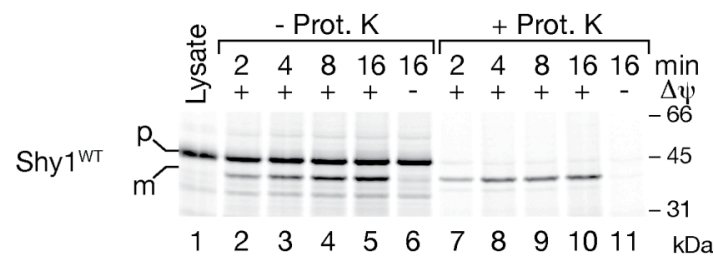

Figure 3.4 In vitro import assay of radiolabeled Shy1

(A) Wild-type and mutant Shyl precursor proteins were synthesized and $\left[{ }^{35} \mathrm{~S}\right]$ labeled in rabbit reticulocyte lysate and analyzed by SDS-PAGE and digital autoradiography. (B) Scheme of an in vitro import assay of $\left[{ }^{35} \mathrm{~S}\right]$ Shyl into isolated yeast mitochondria. (C) Wild-type $\left[{ }^{35} \mathrm{~S}\right]$ Shyl was in vitro imported in mitochondria as depicted in (B) for indicated time-points, with or without membrane potential $(\Delta \psi)$ and proteinase K (Prot.K) treatment. Samples were analyzed by SDS-PAGE and digital autoradiography. Precursor $(\mathrm{p})$ and mature $(\mathrm{m})$ forms of Shy 1 are indicated 
A

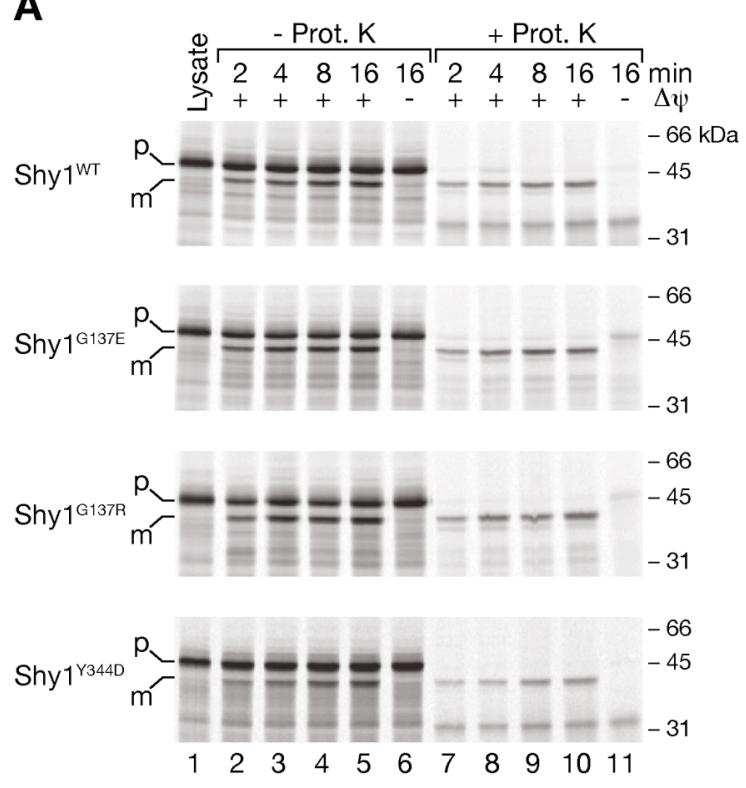

B
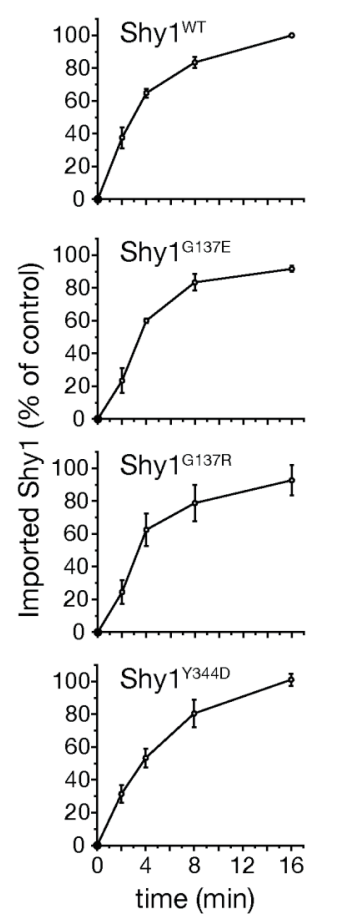

C

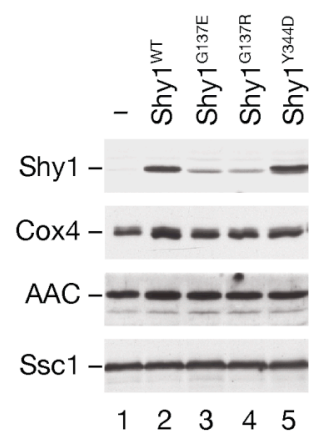

Figure 3.5 Mitochond rial import of mutant Shy1 proteins is not affected

(A) $\left[{ }^{35}\right.$ S]labeled wild-type and mutant Shy1 precursors were in vitro imported into isolated yeast mitochondria in presence or absence of a membrane potential $(\Delta \psi)$ for indicated times. After proteinase $\mathrm{K}$ (Prot.K) treatment, where indicated, samples were analyzed by SDS-PAGE and digital autoradiography. As a standard, 5\% precursor protein (Lysate) was used. p, precursor; m, mature. (B) Quantification of three independent experiments as in (A), using ImageQuant TL (GE-Healthcare). Import efficiency was calculated as protease protected mature Shy1 relative to the input (Lysate). Import efficiency of Shy $1{ }^{\text {WT }}$ after 16 min was set to $100 \%$. (SEM, n=3). (C) Whole-cell extracts of indicated strains were analyzed by SDS-PAGE and Western blotting.

from protease treatment, whereas the Shyl precursor was degraded (Fig 3.4C). Subsequently, this assay was used to compare the import efficiency of wild-type and mutant Shy1.

Surprisingly, mutant Shyl proteins were in vitro imported in a comparable manner into isolated yeast mitochondria (Fig 3.5A). This finding was confirmed by a quantitative import assessment of the Shy1 variants, which revealed identical import efficiency and kinetics (Fig 3.5B). In addition, it was shown in whole cell extracts from the different mutants, that no Shyl precursor accumulated in the cytosol, although mature Shy1 was reduced in shy $1^{G 137 E}$ and shy $1^{G 137 R}$ cells (Fig 3.5C) in consistence with the reduction shown in mitochondria, isolated from these cells (Fig 3.3). Hence, it can be excluded that an impaired import of Shy $1^{\text {G137E }}$ and Shy $1^{\text {G137R }}$ accounts for the reduced abundance of this proteins in mitochondria. 
A

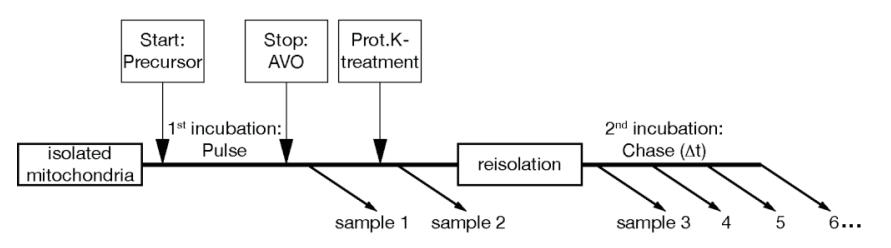

C

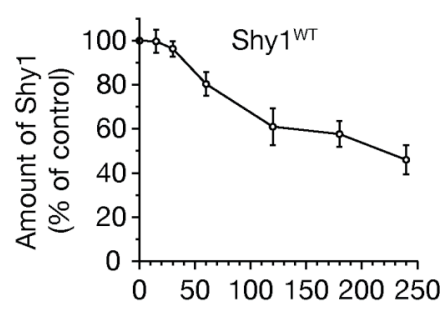

B

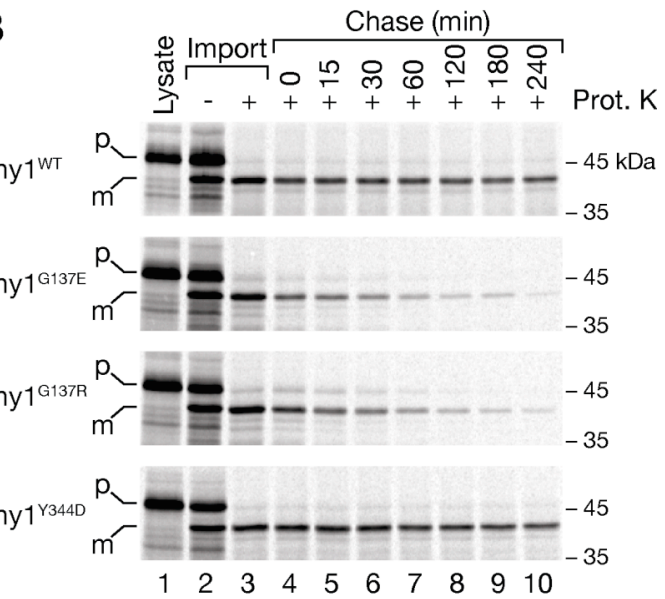

Figure 3.6 Shy $1^{\mathrm{G} 137 \mathrm{E}}$ and Shy ${ }^{\mathrm{G} 137 \mathrm{R}}$ are destabilized in yeast mitochondria

(A) Experimental setup of the stability assay after in vitro import. (B) As shown in (A), $\left[{ }^{35}\right.$ S $]$ labeled Shy 1 precursors were imported in yeast mitochondria for $5 \mathrm{~min}$ (import). Import was stopped, unimported precursor protein (p) was degraded by proteinase $\mathrm{K}$ treatment (Prot.K) and mitochondria were reisolated and resuspended in energized buffer. After further incubation for different times (chase), samples were taken and analyzed by SDS-PAGE and digital autoradiography. m, mature. (C) Three independent experiments as in (B) were quantified using ImageQuant TL (GE-Healthcare). Values represent means of mature Shy1 signals after the indicated time of chase relative to 0 min chase $(=100 \%)$. SEM $(n=3)$.

\subsubsection{Mutation of $\mathbf{G}^{137}$ in Shy1 leads to rapid turnover after import}

The hypothesis of a destabilization of Shy 1 due to the exchange of $\mathrm{G}^{137}$ was analyzed by pulse-chase analysis after in vitro import: Wild-type or mutant $\left[{ }^{35} \mathrm{~S}\right]$ Shy1 was imported into isolated mitochondria, precursor proteins were degraded by protease treatment and samples were taken after different times of further incubation (Fig 3.6A). Although Shy $1^{\text {WT }}$ and Shy $1^{\text {Y344D }}$ were stable, the signal intensity of Shy $1^{\text {G137E }}$ and Shy $1^{\text {G137R }}$ strongly decreased over time (Fig 3.6B). Quantitative analyses of these experiments revealed a half-life of about 4 hours of Shy $1^{\mathrm{WT}}$ and Shy $1^{\mathrm{Y} 344 \mathrm{D}}$ in mitochondria, whereas Shy $1^{\mathrm{G} 137 \mathrm{E}}$ and Shy $1^{\mathrm{G} 137 \mathrm{R}}$ were turned over with a half-life of less than $30 \mathrm{~min}$ (Fig 3.6C). Consequently the reduced 
A

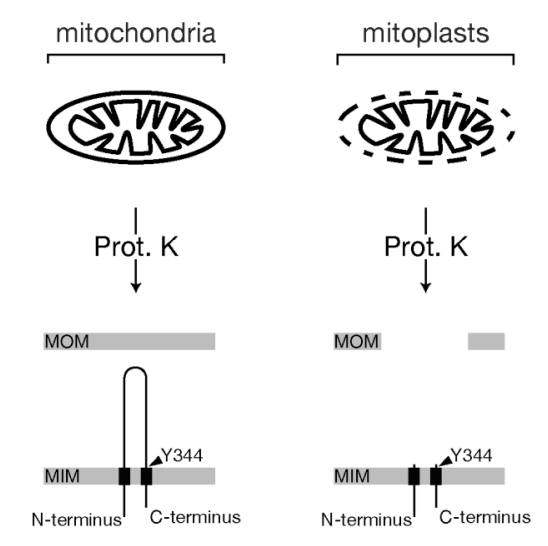

B

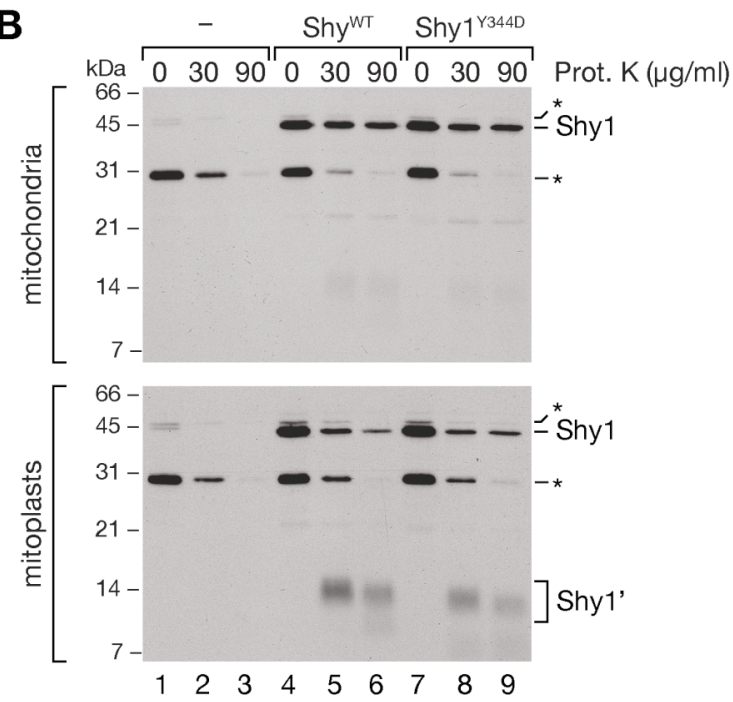

Figure 3.7 Shy $1^{\mathrm{Y} 344 \mathrm{D}}$ is correctly inserted in the mitochondrial inner membrane

(A) Scheme of the experiment shown in (B). (B) From indicated strains, mitochondria or mitoplasts, generated by osmotic swelling, were incubated with increasing concentrations of proteinase K (Prot.K) for $10 \mathrm{~min}$ on ice. Samples were analyzed by SDS-PAGE and Western blotting using antiserum against the C-terminus of Shy1. Asterisk, cross reactive band; Shy1', C-terminal Shy1 fragment; MOM, mitochondrial outer membrane; MIM, mitochondrial inner membrane.

abundance of Shy1 ${ }^{\mathrm{G} 137 \mathrm{E}}$ and Shy $1^{\mathrm{G} 137 \mathrm{R}}$ in mitochondria can be explained by their rapid degradation after mitochondrial import.

\subsubsection{Shy $1^{\mathrm{Y} 344 \mathrm{D}}$ maintains the physiologically correct topology}

It could be shown that Shy $1^{\mathrm{Y} 344 \mathrm{D}}$ is imported into mitochondria and remains stable. However, since the mutation affects an amino acid at the interface of the second transmembrane span, it was hypothesized that the insertion of this transmembrane span into the inner mitochondrial membrane could be impaired. To assess the topology of the second transmembrane span, a protease protection analysis of the C-terminal domain of Shy1 was established (Fig 3.7A): Mitochondria and mitoplasts, generated by osmotic swelling, were incubated with different amounts of protease. Samples were analyzed by SDS-PAGE and Western blotting, using an antibody raised against the C-terminus of Shy1. In mitochondria, Shy1 was protected from protease treatment by the outer mitochondrial membrane (Figure 3.7B). However, in mitoplasts, Shy $1^{\mathrm{WT}}$ and Shy ${ }^{\mathrm{Y} 34 \mathrm{D}}$ were degraded by the protease. Interestingly, in both cases C-terminal domains were detected, which remained protected by the mitochondrial inner membrane, indicating a similar localization in the mitochondrial matrix. Therefore it can be 
A

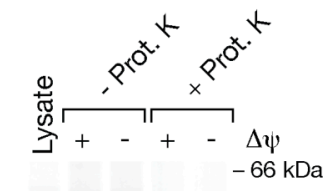

ОтС

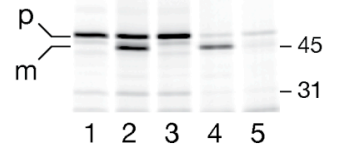

B

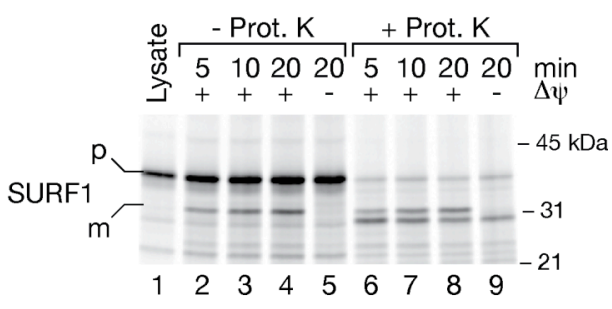

Figure 3.8 In vitro import of SURF1 into human mitochondria

(A) OTC precursor protein was in vitro synthesized and $\left.{ }^{35} \mathrm{~S}\right]$ labeled in reticulocyte lysate (Lysate). In vitro import into isolated HEK293T mitochondria for $20 \mathrm{~min}$ was performed in presence or absence of membrane potential $(\Delta \psi)$ and subsequent proteinase $\mathrm{K}$ (Prot.K) treatment where indicated. (B) $\left[{ }^{35} \mathrm{~S}\right] \mathrm{SURF} 1$ was in vitro synthesized and imported in HEK293T mitochondria for indicated times with and without membrane potential and subsequent proteinase K treatment. Samples of (A) and (B) were analyzed by SDS-PAGE and digital autoradiography. p, precursor; m, mature.

concluded that the exchange of $\mathrm{Y}^{344}$ of Shy1 does not affect the correct physiological topology of the protein.

\subsubsection{Import and stability of SURF1 in human mitochondria}

\subsubsection{Establishment of an in vitro import assay of SURF1}

Considering the destabilization of Shy $1^{\mathrm{G} 137 \mathrm{E}}$ and Shy $1^{\mathrm{G} 137 \mathrm{R}}$ in yeast mitochondria, it was speculated that SURF1 $1^{\mathrm{G} 124 \mathrm{E}}$ and SURF1 ${ }^{\mathrm{G} 124 \mathrm{R}}$ could also be rapidly degraded in human mitochondria. Destabilization of SURF1 in affected patients, similar to a SURF1 deletion in other individuals, would explain the development of Leigh Syndrome.

To confirm a destabilization of SURF1 $1^{\mathrm{G} 124 \mathrm{E} / \mathrm{G} 124 \mathrm{R}}$ in human mitochondria, an in vitro import assay was established for mitochondria, isolated from cultured HEK293T cells. To optimize in vitro import conditions, ornithine transcarbamylase (OTC), a well characterized substrate for in vitro import into mammalian mitochondria (Sztul et al., 1987), was used (Fig 3.8A). Upon incubation of $\left[{ }^{35} \mathrm{~S}\right] \mathrm{OTC}$ precursor with isolated human mitochondria, the faster migrating mature form of OTC accumulated at a protease protected location, dependent on the membrane potential. To use this in vitro import assay for SURF1, the open reading frame of SURF1 was cloned, $\left[{ }^{35} \mathrm{~S}\right] \mathrm{SURF} 1$ was synthesized in reticulocyte lysate and imported in isolated HEK293T mitochondria using the same conditions (Fig 3.8B). Similar to Shy1, also the SURF1 precursor was processed to the mature form, which accumulated in mitochondria dependent on a membrane potential. 


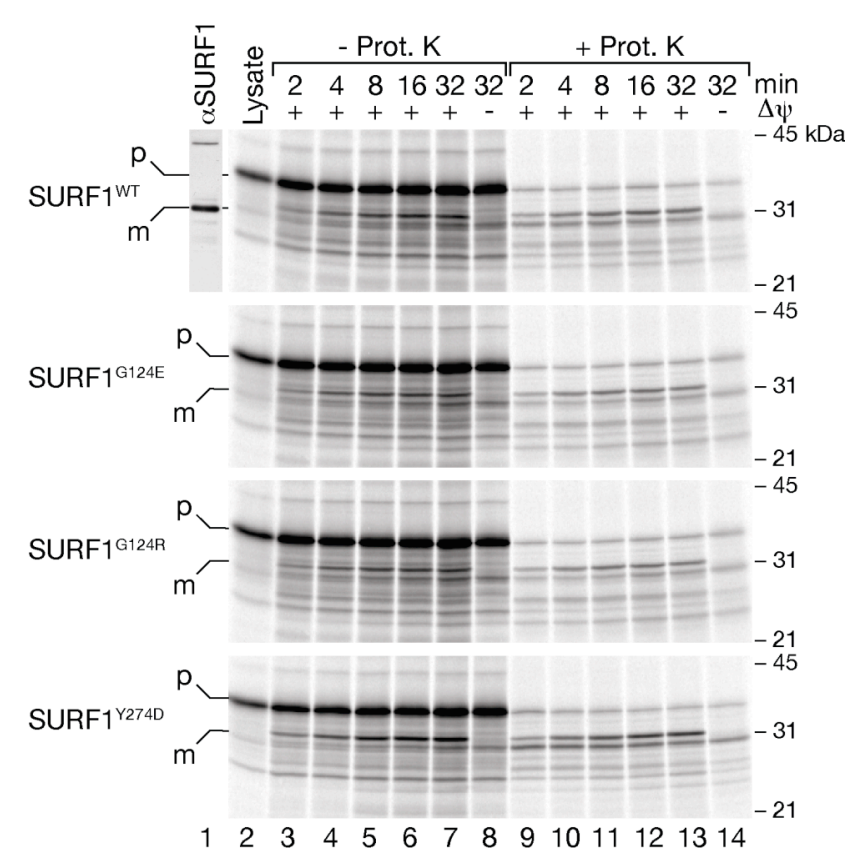

Figure 3.9 Mutant SURF1 imports efficiently into human mitochondria

In vitro import of wild-type and mutant SURF1 precursors (p) into isolated HEK293T mitochondria for indicated times in presence or absence of membrane potential $(\Delta \psi)$. After proteinase $\mathrm{K}$ (Prot.K) treatment (were indicated), samples were subjected to SDS-PAGE and digital autoradiography. As a marker mature (m) SURF1 was visualized by SDS-PAGE and Western blotting (lane 1).

\subsubsection{Mutant SURF1 is efficiently imported into mitochondria}

The import efficiency of the mutant variants of SURF1 was analyzed by in vitro import analyses. Therefore the patient point mutations were introduced into the cloned SURF1 using site directed mutagenesis. Subsequently, $\left[{ }^{35} \mathrm{~S}\right]$ labeled wild-type and mutant SURF1 proteins were synthesized and in vitro import was performed into HEK293T mitochondria (Fig 3.9). Similar to the wild-type protein, SURF1 ${ }^{\mathrm{G} 124 \mathrm{E}}$, SURF1 ${ }^{\mathrm{G} 124 \mathrm{R}}$ and SURF1 ${ }^{\mathrm{Y} 274 \mathrm{D}}$ were processed to the mature form upon incubation with mitochondria and accumulated at a protease protected location dependent on the membrane potential. Moreover, quantification of the signals revealed that the import efficiency was in a range of $\pm 10 \%$ of the wild-type protein (not shown). Hence, it was concluded that similar to the yeast counterparts, the mutant SURF1 proteins are efficiently imported into human mitochondria. 


\subsubsection{Mutations affecting $\mathrm{G}^{124}$ in SURF1 lead to degradation of the mature protein}

Since the mutant SURF1 proteins are efficiently imported into mitochondria, it was speculated that similar to the yeast models, an exchange of $\mathrm{G}^{124}$ in SURF1 leads to destabilization of the protein.

Therefore, the stability of SURF1 proteins, affected by the patient point mutations, was compared with the wild-type protein. To analyze this, the degradation of in vitro imported

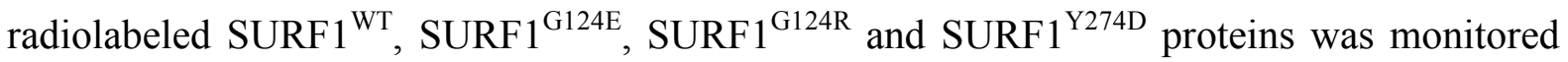
by pulse-chase analysis (Fig3.10A).

Similar to the yeast model, mature SURF $1^{\mathrm{WT}}$ and SURF1 ${ }^{\mathrm{Y} 274 \mathrm{D}}$ remained stable after in vitro import into mitochondria, degradation of the precursor and further incubation for up to 4 hours (Fig 3.10A upper and lower panel). In contrast, SURF1 $1^{\mathrm{G} 124 \mathrm{E}}$ and SURF1 ${ }^{\mathrm{G} 124 \mathrm{R}}$ signals drastically decreased over time. In fact, at incubation times of more than $60 \mathrm{~min}$, signals of SURF1 ${ }^{\mathrm{G} 124 \mathrm{E}}$ and SURF1 ${ }^{\mathrm{G} 124 \mathrm{R}}$ were hardly detectable (Fig 3.10A middle panels).

A quantitative analysis of these experiments revealed a half-life of mature SURF1 ${ }^{\mathrm{WT}}$ and SURF1 ${ }^{\text {Y274D }}$ of more than 4 hours. On the other hand, SURF1 ${ }^{\text {G124E }}$ and SURF1 ${ }^{\text {G124R }}$ were rapidly degraded with half-lifes of about $10 \mathrm{~min}$ (Fig 3.10B).

Taken together, it can be concluded that mutations, affecting the highly conserved amino acid $\mathrm{G}^{124}$ of SURF1, lead to destabilization of the protein, similar to mutations affecting the corresponding $\mathrm{G}^{137}$ of the yeast model Shyl. Exchanges of the highly conserved $\mathrm{Y}^{274}$ in SURF1 ( $\mathrm{Y}^{344}$ in Shy1) however, do not alter the stability of the protein. 
A

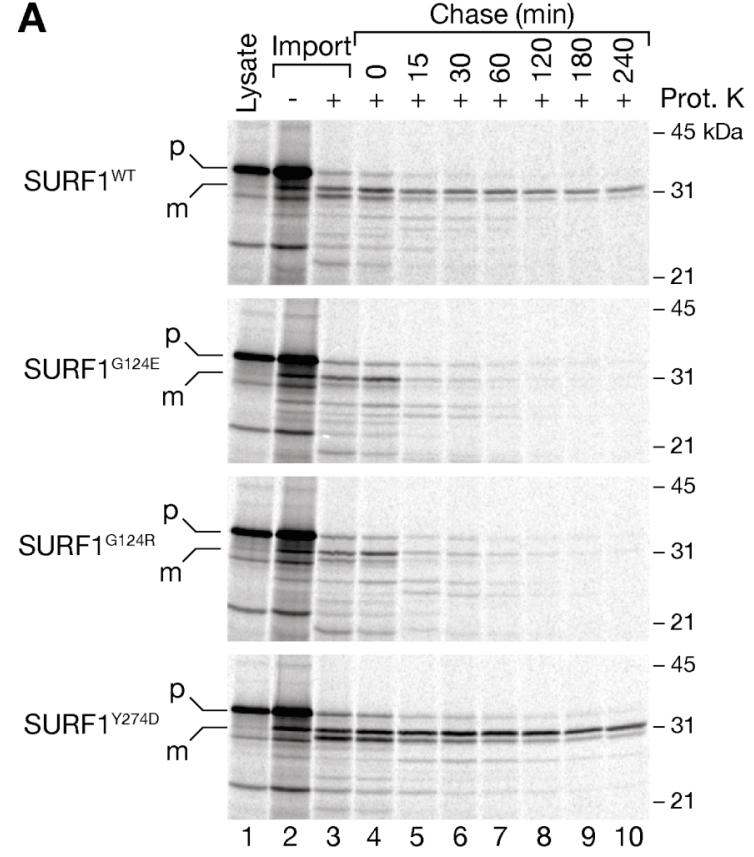

B

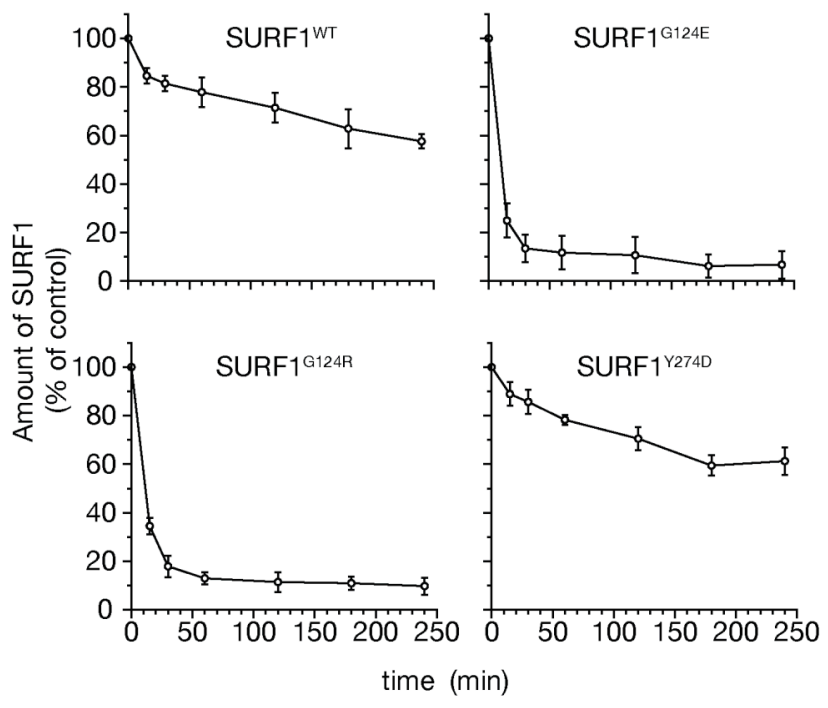

Figure 3.10 Exchange of $G^{124}$ in SURF1 leads to rapid degradation in mitochondria

(A) Stability assay of wild-type and mutant SURF1 after in vitro import as depicted in (Fig 6A): $\left[{ }^{35}\right.$ S]SURF1 precursors ( $p$ ) were in vitro imported into HEK293T mitochondria, after proteinase $\mathrm{K}$ (Prot.K) treatment, mitochondria were reisolated and further incubated for indicated times (chase). Samples were analyzed by SDS-PAGE and digital autoradiography. m, mature. (B) Three independent experiments as in (A) were quantified using ImageQuant TL (GE-Healthcare). Shown are mean ratios of SURF1 signal after indicated times of chase relative to 0 min chase $(=100 \%)$. SEM $(n=3)$.

\subsubsection{SURF1 ${ }^{\mathrm{Y} 274 \mathrm{D}}$ association with cytochrome $c$ oxidase assembly intermediates is affected}

It could be shown that mutations affecting $\mathrm{G}^{124}$ of SURF1 lead to rapid degradation of the protein in mitochondria, which fully explains the cytochrome $c$ oxidase deficiency in the

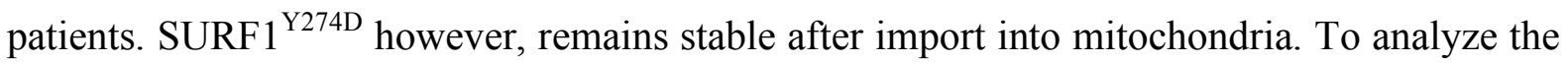
molecular pathology of SURF1 ${ }^{\mathrm{Y} 274 \mathrm{D}}$ in more detail, the assembly of SURF1 to inner mitochondrial membrane complexes was examined. Therefore, protein complexes of isolated HEK293T mitochondria were analyzed by BN-PAGE analysis and Western blotting (Fig 3.11A). Interestingly, using 1\% digitonin as a detergent for solubilization, two SURF1 containing complexes of about 100 and $200 \mathrm{kDa}$ were resolved (Fig 3.11A lane 2). In contrast, mature cytochrome $c$ oxidase migrated as a complex of approximately $400 \mathrm{kDa}$ (Fig 3.11A lanes 3-5). Moreover, COX1-containing putative assembly intermediates were 
also detected at about $200 \mathrm{kDa}$ (Fig 3.11A lane 3), which apparently did not co-migrate with the $200 \mathrm{kDa}$ SURF1 complex.

To analyze this in more detail, the assembly of in vitro imported $\left[{ }^{35} \mathrm{~S}\right] \mathrm{SURF} 1$ was examined (Fig 3.11B). Dependent on the membrane potential, SURF $1^{\mathrm{WT}}$ was imported and assembled into complexes of similar size as seen in the Western blot analysis. Interestingly, minute amounts of SURF $1^{\text {WT }}$ were also detected at the size of the mature cytochrome $c$ oxidase, which was visualized by the assembly of COX6A1 (Fig 3.11B lane 5). In contrast, assembly analysis of the mutant SURF1 ${ }^{\mathrm{Y} 274 \mathrm{D}}$ revealed a preferential association to the $200 \mathrm{kDa}$ complex (Fig 3.11C). Essentially no assembly of SURF1 ${ }^{\mathrm{Y} 274 \mathrm{D}}$ into the $100 \mathrm{kDa}$ complex or into the mature cytochrome $c$ oxidase could be detected.

Subsequently, the assembly of SURF $1^{\text {WT }}$ and SURF1 ${ }^{\mathrm{Y} 274 \mathrm{D}}$ was directly compared and the nature of the observed SURF1 complexes was addressed in mitochondria, depleted of cytochrome $c$ oxidase assembly intermediates (Fig 3.11D). Therefore, mitochondrial or cytosolic translation was inhibited by chloramphenicol (CAP) or cycloheximide (CHX) respectively, prior to isolation of mitochondria. Interestingly, although SURF1 ${ }^{\mathrm{WT}}$ and SURF1 ${ }^{\mathrm{Y} 274 \mathrm{D}}$ import was undistinguishable (Fig 3.11D lower panel), the assembly of SURF1 ${ }^{\mathrm{Y} 274 \mathrm{D}}$ into the $200 \mathrm{kDa}$ complex was strongly increased compared to SURF1 ${ }^{\mathrm{WT}}$ (Fig 3.11D lane 2 and 6). Moreover, compared to untreated mitochondria, in mitochondria treated with CAP, the assembly of SURF $1^{\mathrm{WT}}$ and SURF1 ${ }^{\mathrm{Y} 274 \mathrm{D}}$ into the $200 \mathrm{kDa}$ complex was strongly decreased (Fig 3.11D lane 3 and 7). This result indicates that the assembly of SURF1 into the $200 \mathrm{kDa}$ complex depends on mitochondrial translation and most likely assembly intermediates of the cytochrome $c$ oxidase.

Taken together it could be shown that SURF1 assembles to putative cytochrome $c$ oxidase assembly intermediates, which are uncharacterized so far. Interestingly an $\mathrm{Y}^{274} \mathrm{D}$ exchange in SURF1 leads to an altered association of the protein to these complexes. 
A

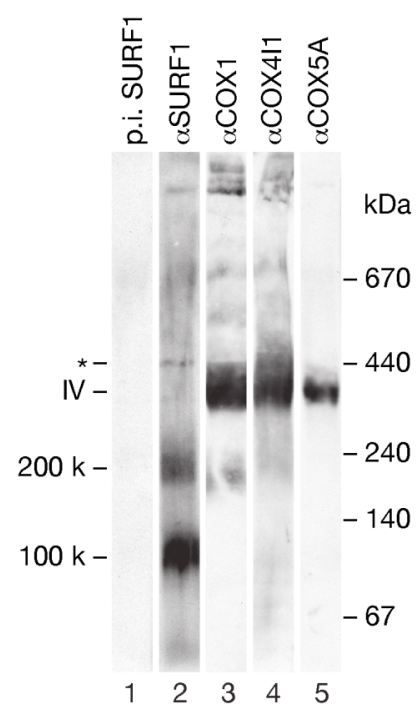

C

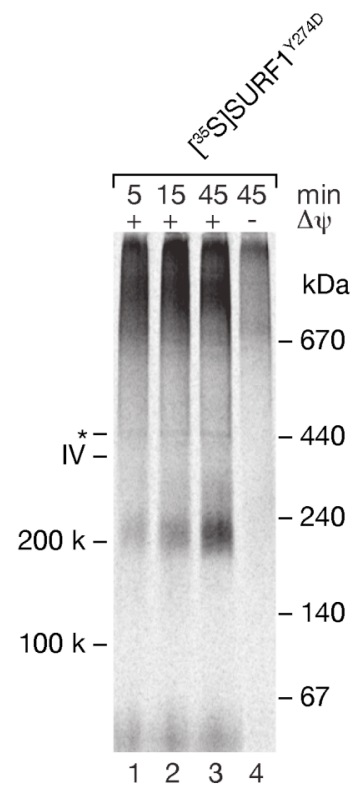

B

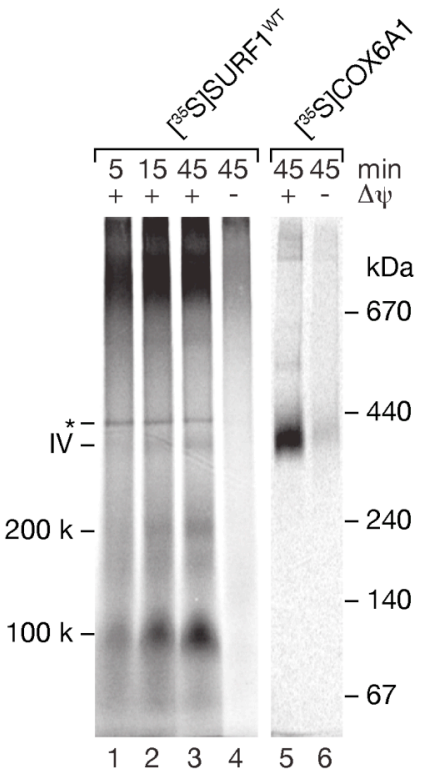

D
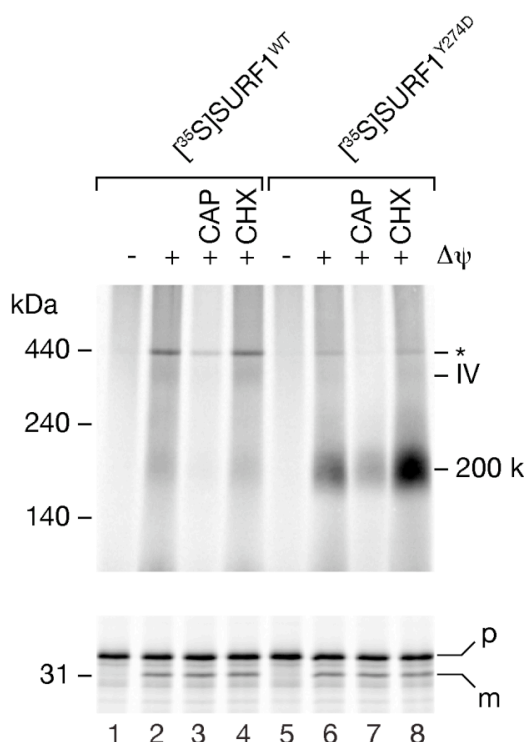

Figure 3.11 Altered association of SURF1 ${ }^{\mathrm{Y} 274 \mathrm{D}}$ to cytochrome $c$ oxidase assembly intermediates

(A) Mitochondrial protein complexes of HEK293T cells were solubilized in 1\% digitonin and analyzed by BN-PAGE and Western blotting using indicated antibodies or preimmune serum (p.i.). (B, C) $\left[{ }^{35}\right.$ S $]$ labeled SURF $1^{\mathrm{WT}}$ and COX6A1 (B) or SURF $1{ }^{\mathrm{Y} 274 \mathrm{D}}$ (C) were in vitro imported into HEK293T mitochondria for indicated times in presence or absence of a membrane potential $(\Delta \psi)$. Subsequently, complexes were solubilized in 1\% digitonin and analyzed by BN-PAGE and digital autoradiography. (D) In vitro import of SURF $1^{\mathrm{WT}}$ or SURF $1^{\mathrm{Y} 274 \mathrm{D}}$ for $45 \mathrm{~min}$ into mitochondria, isolated from chloramphenicol (CAP), cycloheximide (CHX) or untreated HEK293T cells. Subsequently, samples were split and analyzed by SDS-PAGE (lower panel) or BN-PAGE (upper panel) followed by digital autoradiography. Asterisk, frequently observed unspecific protein band; $p$, precursor; $m$, mature. 


\subsubsection{Shy $1^{\text {Y344D }}$ uncouples Cox1 expression from cytochrome $c$ oxidase assembly}

\subsubsection{Shy $1^{\mathrm{Y} 344 \mathrm{D}}$ permits Cox1 expression}

In contrast to SURF1 ${ }^{\mathrm{WT}}$, SURF1 ${ }^{\mathrm{Y} 274 \mathrm{D}}$ accumulates preferentially in a $200 \mathrm{kDa}$ complex. The effects of this accumulation apparently lead to lack of cytochrome $c$ oxidase, as shown in affected patients (Pequignot et al., 2001; Teraoka et al., 1999). The exact role of SURF1 in cytochrome $c$ oxidase assembly remains enigmatic. However, using its yeast homolog as a model, it could be shown that Shyl couples cytochrome $c$ oxidase assembly to translational regulation of its central subunit Cox1 (Barrientos et al., 2002; Mick et al., 2007).

Hence, it was speculated that Cox 1 translational regulation is affected by the mutations in SHY1. This would also explain the reduced steady state levels of Cox1 in cells expressing mutant Shy1 (Fig 3.3). Therefore, mitochondrial translation was analyzed by in vivo labeling of mitochondria-encoded proteins (Fig 3.12A). As reported previously (Barrientos et al., 2002), deletion of SHY1 leads to strongly reduced expression of Cox1. As expected, in cells

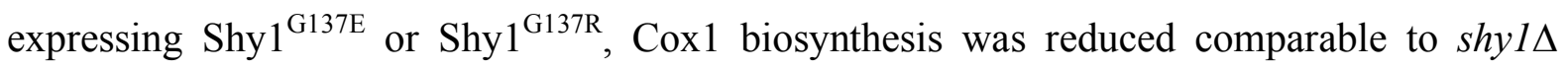
cells due to the rapid degradation of the mutant Shy1 proteins. However, cells expressing Shy $1^{\text {Y344D }}$ displayed Cox1 expression, which was indistinguishable from wild-type cells (Fig 3.12 A and B).

In addition, it could be shown that even at non-permissive temperature $\left(19^{\circ} \mathrm{C}\right)$ shyl $1^{Y 344 D}$ cells showed Cox1 expression that was similar to wild-type cells (Fig 3.12 C and D). 

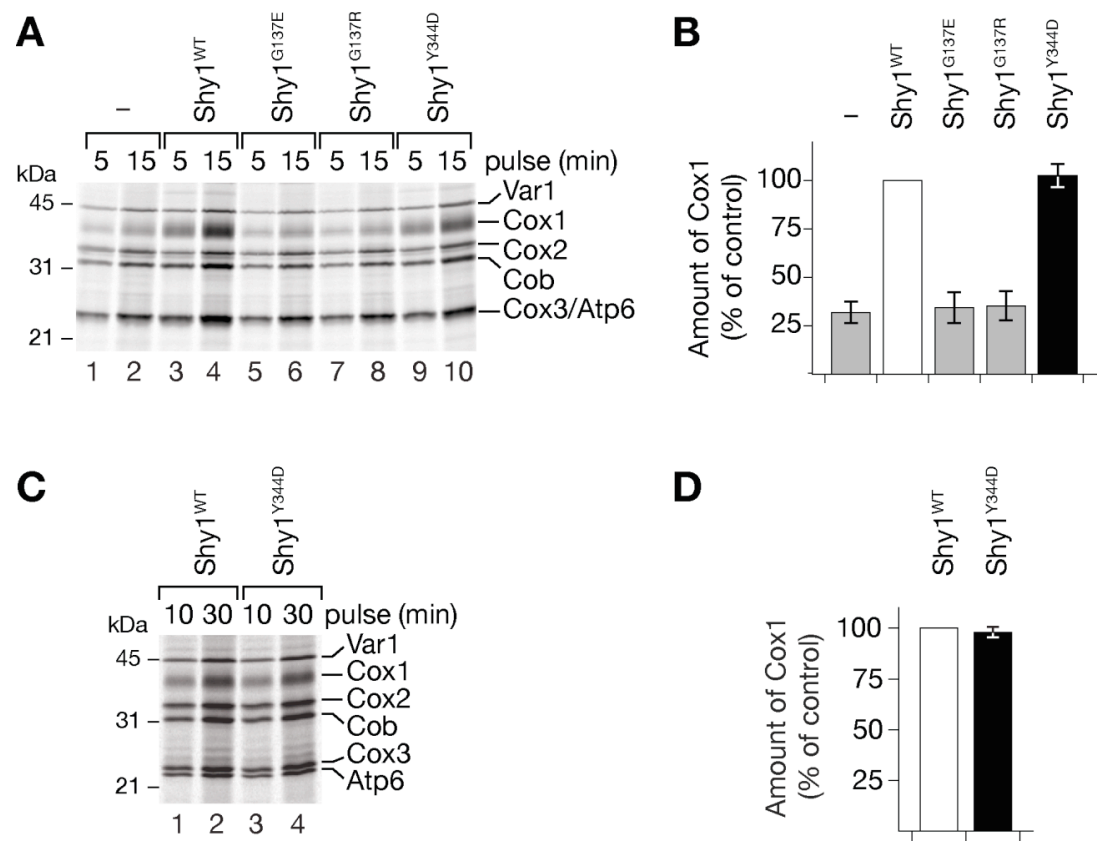

Figure 3.12 shy $1^{\text {Y344D }}$ cells efficiently translate mitochondria-encoded proteins

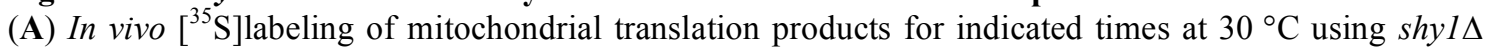
cells, grown at $30^{\circ} \mathrm{C}$, expressing wild-type or mutant Shy1 or containing an empty vector (-). Subsequently, whole cell extracts were prepared and analyzed by SDS-PAGE and digital autoradiography. (B) Signals of three independent experiments as in (A) were quantified using ImageQuant TL (GE-Healthcare). Bars represent mean ratios of Cox1/Cob after 15 min labeling relative to the wild-type $(=100 \%)$. SEM $(n=3)$. Cox1 signals were normalized to Cob, which is expressed independent of defective cytochrome $c$ oxidase assembly $(\mathbf{C}, \mathbf{D})$ shyl $1 \Delta$ cells, expressing Shy $1^{\text {WT }}$ or Shy $1^{\mathrm{Y} 344 \mathrm{D}}$, were grown at $19^{\circ} \mathrm{C}$ and in vivo labeling (C) was performed as in (A) but at $19^{\circ} \mathrm{C}$. Quantification (D) of the signals after 30 min labeling was performed as in (B). SEM ( $n=3)$.

Hence, in shy $1^{Y 344 D}$ cells, Cox1 expression is unaffected although assembly of the cytochrome $c$ oxidase is impaired. Therefore, Cox1 biosynthesis is apparently uncoupled from translational feedback regulation in $s h y 1^{Y 344 D}$ mitochondria.

\subsubsection{Shy $1^{\mathrm{Y} 344 \mathrm{D}}$ releases Cox1 from translational feedback regulation}

Interestingly, in $s h y 1^{Y 344 D}$ mitochondria, the Cox 1 translational feedback regulation system is non-functional: Although cytochrome $c$ oxidase assembly is defective, Cox 1 translation is not shut down.

To address Cox 1 translational regulation in shyl $1^{Y 344 D}$ mitochondria, early cytochrome $c$ oxidase assembly intermediates were analyzed, which are known to link to feedback regulation of Cox 1 translation. These assembly intermediates, containing Coa1, Coa3 and Mss51, were analyzed by co-immunoprecipitation analyses of Coa1 (Fig 3.13A) and Coa3 (Fig 3.13B). 
A

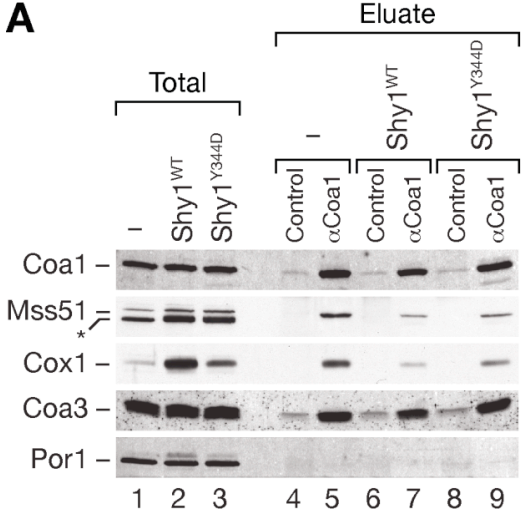

B

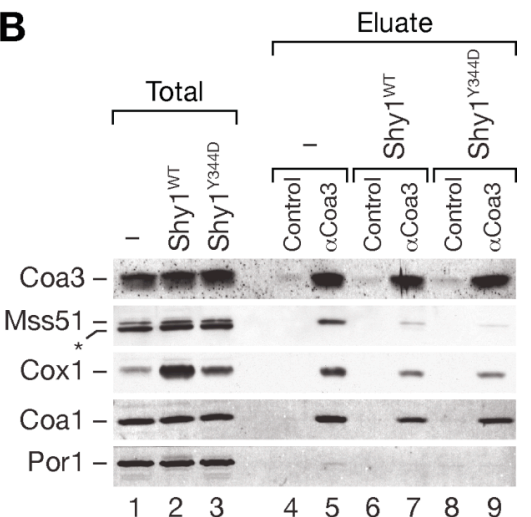

Figure 3.13 Analysis of early cytochrome $c$ oxidase assembly intermediates in yeast

(A, B) Co-immunoprecipitation of Coa1 (A) or Coa3 (B) from mitochondria, solubilized in 1\% digitonin, isolated from shy $1 \Delta$ cells, expressing Shy $1^{\mathrm{WT}}$, Shy $1^{\mathrm{Y} 344 \mathrm{D}}$ or containing an empty vector (-): Lysates were incubated with specific or control antibodies. Bound proteins were eluted after extensive washing and analyzed by SDS-PAGE and Western blotting. Total, 6\%; eluate, 100\%; Asterisk, cross-reactive band.

Antibodies, directed against Coal, efficiently precipitated Coal in shyl 1, shyl ${ }^{Y 344 D}$ and wildtype mitochondria (Fig 3.13A). Interestingly, compared to the wild-type, in shyls mitochondria increased amounts of Cox1 and Mss51 were co-precipitated, indicating an accumulation of cytochrome $c$ oxidase assembly intermediates that shut down Cox1 translation. In contrast, wild-type and shy $1^{Y 344 D}$ mitochondria showed comparable amounts of co-precipitated Cox1 and Mss51, indicating no increased accumulation of these assembly intermediates due to the $\mathrm{Y}^{344} \mathrm{D}$ exchange in Shy1. As a control, Coa3, a known interactor of Coal was detected, and Por1 was analyzed to check for unspecific binding. This finding was confirmed in co-immunoprecipitation experiments, using antibodies directed against Coa3, which also interacts with Cox1 at earlier steps of its biogenesis independent of Coal (Fig 3.13B). Again, an accumulation of early intermediates of cytochrome $c$ oxidase biogenesis, containing Cox1 and Mss51 could be shown in shy1s mitochondria, which were less abundant in wild-type and shyl $1^{Y 344 D}$ mitochondria.

This finding fully explains the wild-type like expression of Cox1 in mitochondria from shy $1^{Y 344 D}$ cells, where no cytochrome $c$ oxidase assembly intermediates accumulate that sequester Mss51 and thereby repress Cox1 translation.

\subsubsection{Cox1 is rapidly degraded in shy $1^{Y 344 D}$ cells}

The wild-type-like expression of Cox 1 in shyl $1^{Y 344 D}$ mitochondria is in contradiction to the observed reduced steady state level of Cox1 (Fig 3.3). Therefore it was speculated that Cox1, 


\section{A}

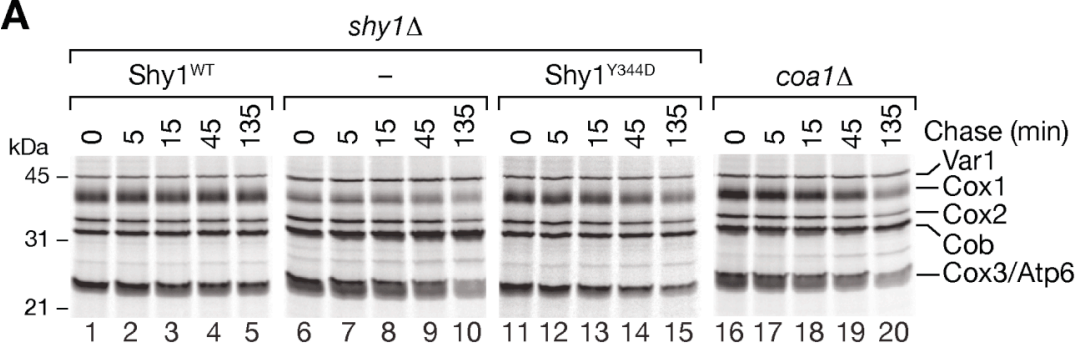

B

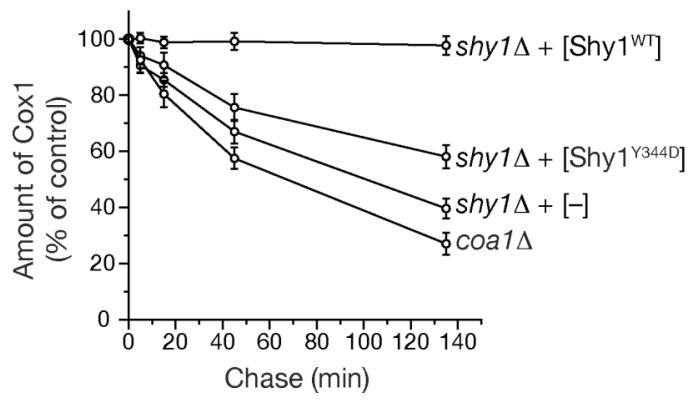

Figure 3.14 Cox1 is rapidly turned over in shy $1^{Y 344 D}$ cells

(A) Mitochondrial translation products of indicated strains were in vivo $\left[{ }^{35} \mathrm{~S}\right]$ labeled for $15 \mathrm{~min}$. After labeling was stopped by addition of $100 \mu \mathrm{g} / \mathrm{ml} \mathrm{CAP}$ and $4 \mathrm{mM}$ unlabeled methionine, incubation was continued at $30^{\circ} \mathrm{C}$. After indicated times of chase, samples were taken, whole cell extracts were prepared and analyzed by SDS-PAGE and digital autoradiography. (B) Experiments as in (A) were quantified (ImageQuant TL, GE-Healthcare) and Cox 1 stability was determined as mean ratios of Cox 1/Cob at each time-point relative to 0 min chase $(=100 \%)$ for the individual strain. SEM $(n=3)$.

that is synthesized in shyl $1^{Y 344 D}$ mitochondria must be rapidly turned over. To test this hypothesis, a stability assay of mitochondrial translation products was established: Mitochondrial translation products were labeled in vivo using $\left[{ }^{35} \mathrm{~S}\right]$ methionine, translation was stopped and degradation was monitored after further incubation of the cells for different times (Fig 3.14A). In wild-type cells, all mitochondrial translation products remained stable and no decrease of signal intensity was observed. However, in shyl $1 \Delta$ and shyl ${ }^{Y 344 D}$ cells, the mitochondria-encoded subunits of the cytochrome $c$ oxidase Cox1, Cox 2 and Cox 3 were degraded.

A quantitative analysis of Cox1 stability in the different strains (Fig 3.14B) revealed that

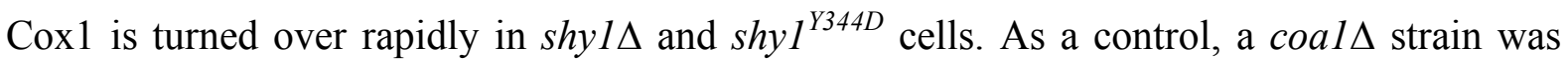
used, which is known to have destabilized Cox1 (Pierrel et al., 2007). This strain showed even faster Cox 1 degradation than shyl $1 \Delta$ or shy $1^{Y 344 D}$ cells. The destabilization of Cox 1 in shy $1^{Y 344 D}$ cells explains the lack of Cox 1 at steady state although Cox1 expression is not affected in this strain. 
A

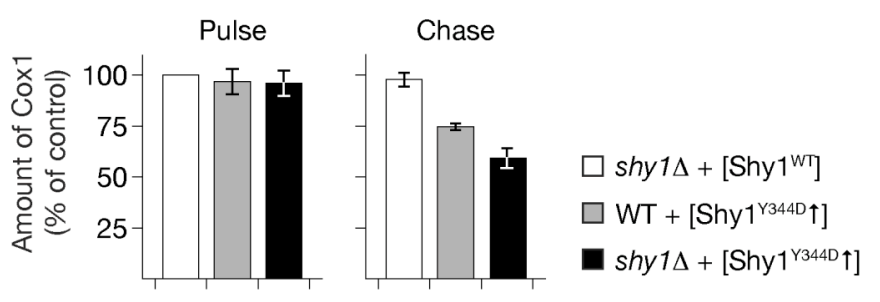

B

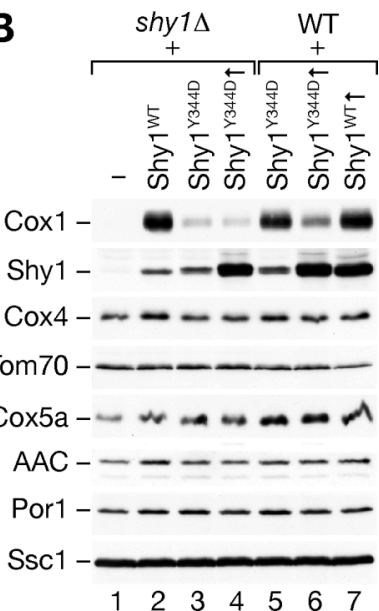

Figure 3.15 Shy $1^{\text {Y344D }}$ has a dominant negative effect on Cox1 stability in yeast

(A) Quantification of Cox1 synthesis (pulse for $15 \mathrm{~min}$ ) and stability (chase for $135 \mathrm{~min}$ ) as analyzed in (Fig 3.14B) in indicated strains. In brackets are the proteins, expressed from a plasmid ( $\uparrow$ indicates overexpression). (B) From wild-type or shyls cells, expressing indicated proteins from a plasmid ( $\uparrow$ indicates overexpression) whole cell extracts were prepared and analyzed by SDS-PAGE and Western blotting.

\subsubsection{Shy $1^{\mathrm{Y} 344 \mathrm{D}}$ has a dominant negative effect on Cox1 stability}

It could be shown that Shy $1^{\mathrm{Y} 34 \mathrm{D}}$ is stable after mitochondrial import but is nonfunctional and leads to rapid Cox1 degradation due to defective cytochrome $c$ oxidase assembly. To test if Shy $1^{\mathrm{Y} 344 \mathrm{D}}$ has a dominant negative effect, the open reading frame encoding for Shy $1^{\mathrm{Y} 344 \mathrm{D}}$ was cloned in a multicopy vector, which was transformed in wild-type and shyls cells. Growth analyses of these strains did not show a dominant effect (not shown). However, pulse-chase experiments after $\left[{ }^{35} \mathrm{~S}\right]$ labeling of mitochondrial translation products revealed a destabilization of Cox1 in wild-type cells, which overexpress Shy $1^{\text {Y344D }}$ (Fig 3.15A). To confirm this dominant negative effect, steady-state levels of Cox1 were analyzed in strains overexpressing Shy $1^{\mathrm{Y} 344 \mathrm{D}}$ in shyld and wild-type background (Fig 3.15B). In agreement with the pulse-chase experiments, it was shown that overexpression of Shy $1^{\mathrm{Y} 344 \mathrm{D}}$ in wild-type background leads to reduced Cox1 levels, whereas Cox1 is little affected when Shy $1^{\mathrm{Y} 344 \mathrm{D}}$ is co-expressed with the wild-type copy in similar amounts. Hence, Shy $1^{\mathrm{Y} 344 \mathrm{D}}$ not only shows lack of function in regard to cytochrome $c$ oxidase assembly, it even has a dominant negative effect on Cox 1 stability when overexpressed in the wild-type background. 


\subsubsection{Mitochondrial quality control by mitophagy}

Cells expressing Shy $1^{\text {Y344D }}$ show wild-type like expression of Cox1. However, Cox1 is rapidly degraded. The degradation of accumulated assembly intermediates of the cytochrome $c$ oxidase plays an essential role for cell viability, since some assembly intermediates are prone to production of reactive oxygen species (Khalimonchuk et al., 2007; Rigoulet et al., 2011). These highly reactive compounds lead to damage of proteins, DNA and lipids. Cells counteract oxidative stress by expressing detoxifying enzymes such as catalase and superoxide dismutase (Jamieson, 1998). Moreover damaged mitochondria can be degraded via selective autophagic degradation of mitochondria (mitophagy) (Kanki and Klionsky, 2010; Tal et al., 2007).

It is conceivable, that in shy $1^{Y 344 D}$ cells, an increased production of reactive oxygen species due to impaired cytochrome $c$ oxidase assembly, triggers mitophagy. This would also explain the rapid Cox 1 turnover observed in shy $1^{Y 344 D}$ mitochondria. The first mitochondrial proteins, reported to be involved in mitophagy are Uth1 and Aup1 (Kissová et al., 2004; Tal et al., 2007). It was hypothesized that Uth1 is a mitochondrial outer membrane receptor for autophagosome formation (Camougrand et al., 2003; Kissová et al., 2004). Therefore it was speculated that in shy $1^{Y 344 D}$ cells, Uth1 could be involved in degradation of mitochondria. Interestingly, there is a controversy about the $\mathrm{N}$-terminus of Uth1: Based on sequence homology to ortholog yeast proteins and mapping of transcription start sites (Kellis et al., 2003; Zhang and Dietrich, 2005) it was suggested, that Uth1 translation starts at the second start-codon of the open reading frame (Fig 3.16A). To analyze its N-terminus and mitochondrial localization, Uth1 starting from the first start codon of the open reading frame was in vitro translated and $\left[{ }^{35} \mathrm{~S}\right]$ labeled using rabbit reticulocyte lysate. Subsequently, in vitro import experiments into isolated yeast mitochondria were performed (Fig 3.16B). Interestingly, $\left[{ }^{35} \mathrm{~S}\right] \mathrm{Uth} 1 \mathrm{was}$ processed to a faster migrating form upon incubation with mitochondria. Moreover, this mature form accumulated in a membrane potential $(\Delta \psi)$ dependent manner and was protected against protease treatment. This is strong evidence that Uth1, with its predicted transmembrane span, in fact is located in the mitochondrial inner membrane. The previously suggested outer membrane localization of Uth1 might be due to mislocalization of the tagged protein used in this study (Velours et al., 2002). Furthermore the potential of $\left[{ }^{35} \mathrm{~S}\right] \mathrm{U}$ th1, starting from the first possible start methionine, to be imported in vitro and its processing led to the hypothesis, that Uth1 translation starts at the first start- 


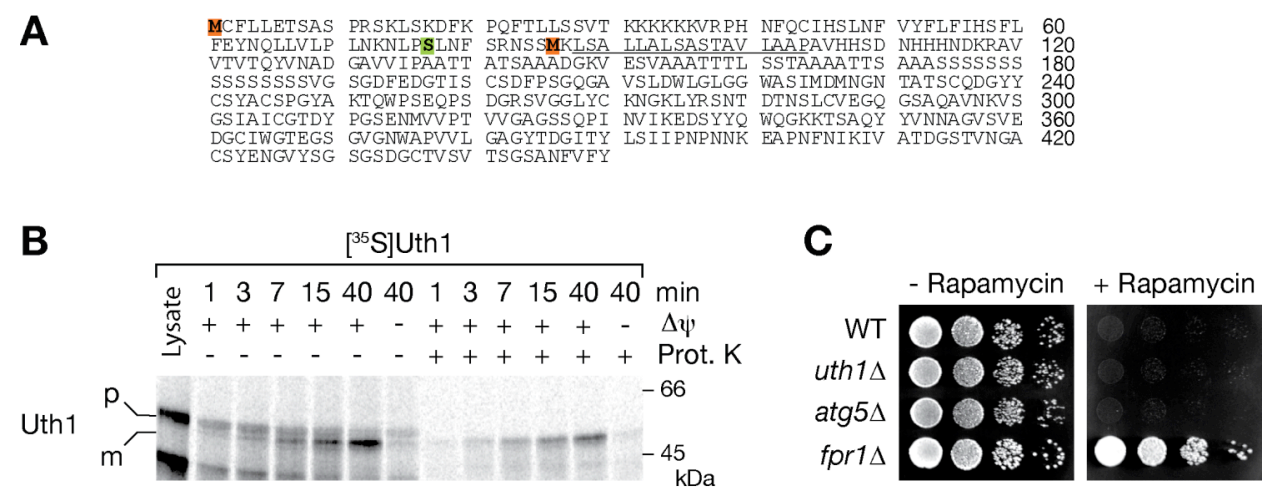

Figure 3.16 Analysis of Uth1, the suggested mitophagy receptor in the outer membrane

(A) Amino acid sequence of Uth1. The potential start-methionines (red), the predicted transmembrane span (underlined) and the N-terminus identified by mass spectrometry (green) are indicated. (B) In vitro import of $\left[{ }^{35} \mathrm{~S}\right] \mathrm{Uth} 1$ into isolated yeast mitochondria in presence or absence of a membrane potential $(\Delta \psi)$ for different times with subsequent proteinase $\mathrm{K}$ (Prot.K) treatment as indicated. The $\left[{ }^{35} \mathrm{~S}\right] \mathrm{Uth} 1$ precursor (Lysate) was used as a standard. p, precursor; m, mature. (C) Serial dilutions of indicated yeast strains were spotted on YPD plates supplemented with $0.2 \mu \mathrm{g} / \mathrm{ml}$ rapamycin if indicated. Plates were incubated for 3 days at $30^{\circ} \mathrm{C}$.

codon of the open reading frame and that the protein is processed to a mature form after mitochondrial import. Interestingly, no N-terminal Uth1 peptide was found in a screen to identify the N-termini of all mitochondrial proteins (Vögtle et al., 2009). Using this data however, an Uth1 peptide was identified in collaboration with Dr. R. Zahedi (Leibniz Institut für Analytische Wissenschaften, Dortmund), which started between the two possible startmethionines (Fig 3.16A). This finding confirms the translation-start of Uth1 at the first potential start methionine and its processing after mitochondrial import.

To study the role of Uth1 in autophagy, the response of uthls cells to rapamycin was analyzed. Previous studies (Kissová et al., 2004) showed a resistance of uth $1 \Delta$ cells to rapamycin, which induces growth defects in wild-type cells by artificial induction of autophagy. In contrast, in the present study no resistance to rapamycin of uth $1 \Delta$ cells (BY4741 background) could be observed (Fig 3.16C). As a control fprls cells were used, which are resistant to rapamycin (Heitman et al., 1991). As an additional control atg5 $\Delta$ cells were analyzed, which are defective of autophagy. These cells do not survive starvation and show reduced viability (Kametaka et al., 1996; Kissová et al., 2004).

Moreover, also a uth1s strain derived from YPH499 was analyzed which also showed no resistance to rapamycin treatment (not shown).

Taken together, it was concluded, that Uth1 is localized to the mitochondrial inner membrane and that the role of Uth1 in autophagic degradation of mitochondria remains questionable. 


\subsection{Sym1, a model for MPV17-associated Mitochondrial DNA Depletion Syndrome}

\subsubsection{Sym1 is a polytopic protein of the mitochondrial inner membrane}

\subsubsection{MPV17 patient mutations affect highly conserved amino acids}

MPV17, a highly conserved mitochondrial inner membrane protein, is involved in the Mitochondrial DNA Depletion Syndrome (Spinazzola et al., 2006). In patients, mutations were identified that lead to an $\mathrm{R}^{50} \mathrm{Q}$ or $\mathrm{N}^{166} \mathrm{~K}$ exchange in MPV17. Interestingly, these amino acids are invariant in homolog proteins (Fig 3.17A) and correspond to $\mathrm{R}^{51}$ or $\mathrm{N}^{172}$ in the yeast ortholog Sym1. Hence, Sym1 can be used as a model to analyze these patient mutations.

Characteristic for MPV17, Sym1 and their homologs are four predicted transmembrane spans (Fig 3.17B), which might be affected in the mutant proteins. The exchange of the basic $\operatorname{arginine}^{51}$ of Sym1 ( $\mathrm{R}^{50}$ in MPV17) to a glutamine residue could influence protein interactions or the insertion of the second predicted transmembrane span (Fig 3.17C). Similarly, the exchange of the asparagine at position 172 in Sym1 ( ${ }^{166}$ in MPV17) to a basic lysine residue in the fourth predicted transmembrane span (Fig 3.17B and C) could also be deleterious for membrane insertion and protein interactions in a hydrophobic environment. In this study, Sym1 was used as a model, to resolve the molecular function and biogenesis of MPV17/Sym1, which is to date unknown.

\subsubsection{SYM1 deletion does not affect respiratory competence in yeast}

Sym1 has been reported to be required for stress tolerance in yeast. Studies by Trott and Morano (2004) and Spinazzola et al. (2006) showed that strains, lacking Sym1, derived from BY4743 or BY4741 respectively, did not grow on ethanol containing media under heat shock conditions. To validate this finding, SYM1 was deleted in YPH499 background and growth tests were performed (Fig 3.18A). As controls, wild-type cells and cells, lacking mitochondrial DNA $\left(r h o^{0}\right)$, were used. As reported previously, syml $\Delta$ cells were able to grow on nonfermentable carbon sources (YPG). However, sym1 $1 \Delta$ cells also grew comparable to wild-type cells on ethanol containing media (YPE) at all temperatures tested. 


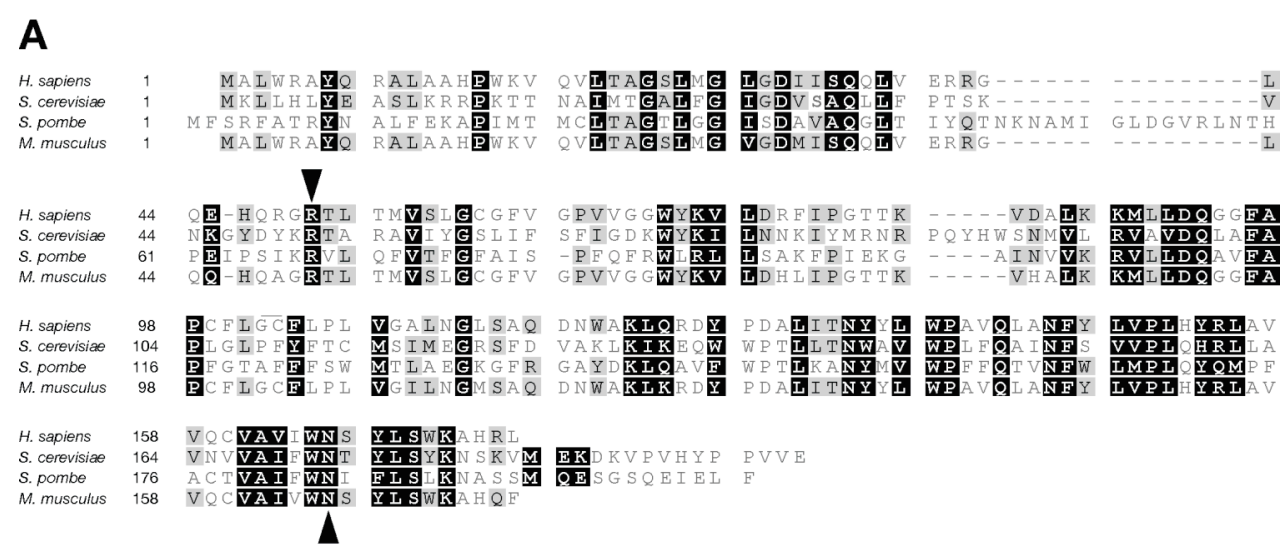

B
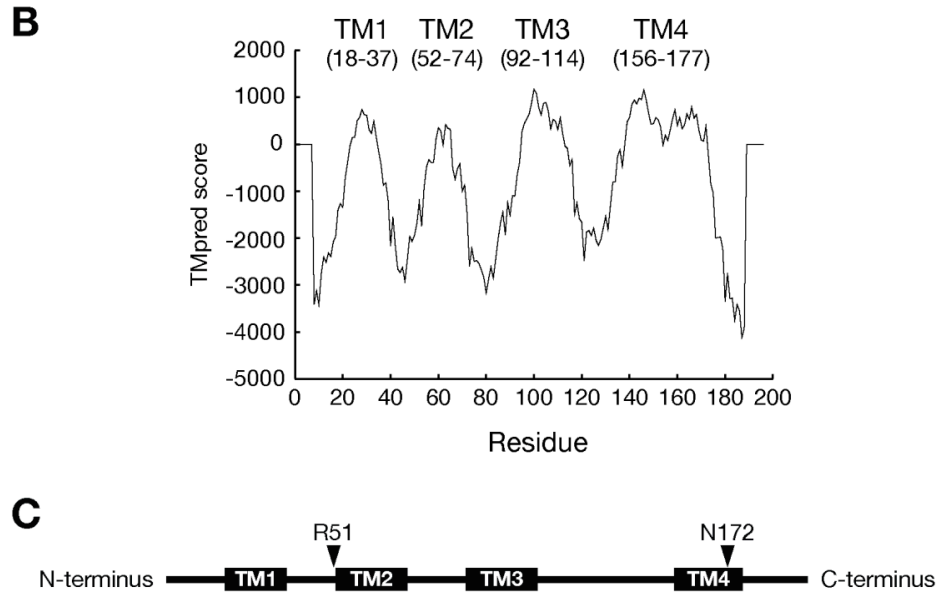

Figure 3.17 Patient mutations in MPV17 affect conserved residues in putative transmembrane spans

(A) Alignment of MPV17 homolog proteins using ClustalW2 (Blosum62 matrix): Black boxes indicate $100 \%$; dark gray $80-100 \%$; light gray $60-80 \%$ similarity. Arrowheads show amino acids affected by patient mutations. (B) Transmembrane span prediction of Sym1 using TMpred. Putative transmembrane (TM) segments are indicated. (C) Predicted domain-structure of Sym1: Transmembrane domains (TM) and patient mutations (arrowheads) are indicated.

Interestingly, altered respiratory chain activities were reported in patients with mutations in MPV17 (Wong et al., 2007). Therefore, steady-state levels of respiratory chain subunits and complexes in isolated mitochondria were analyzed by SDS-PAGE and BN-PAGE (Fig 18B and C). Marker proteins of the outer and inner membrane (Tom70 and Tim23) and the mitochondrial matrix (Pam17, Ssc1) remained unchanged in sym1s mitochondria compared to the wild-type. Moreover, structural subunits and assembly factors of the respiratory chain were indistinguishable in wild-type and $\operatorname{sym} 1 \Delta$ mitochondria (Fig 3.18B), confirming the growth analysis. Furthermore, BN-PAGE analysis of respiratory chain supercomplexes in sym $1 \Delta$ and wild-type mitochondria showed no differences (Fig 3.18C). 
A

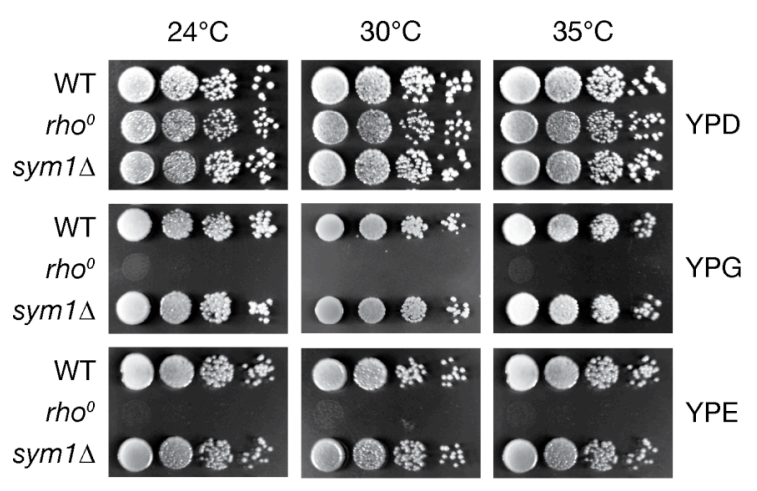

B
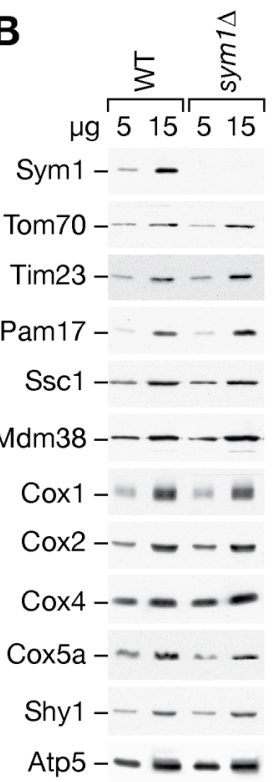

$\begin{array}{llll}1 & 2 & 3 & 4\end{array}$
C

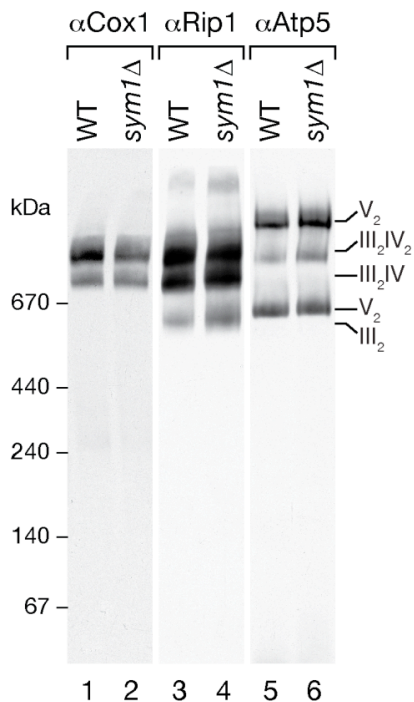

Figure 3.18 Cells lacking Sym1 show respiratory competence

(A) Serial dilutions of wild-type (WT), cells lacking mitochondrial DNA $\left(r h o^{0}\right)$ or $\operatorname{sym} 1 \Delta$ cells were spotted on rich media plates, containing glucose (YPD), glycerol (YPG) or ethanol (YPE) as a carbon source and incubated at the indicated temperatures. (B) Indicated amounts $(\mu \mathrm{g})$ of isolated mitochondria from wild-type or $\operatorname{sym} 1 \Delta$ cells were analyzed by SDS-PAGE and Western blotting with antibodies as indicated. (C) Wild-type or sym $1 \Delta$ mitochondria were solubilized and respiratory chain complexes were analyzed by BN-PAGE and Western blotting.

Hence, no respiratory phenotype and altered respiratory chain composition in $s y m 1 \Delta$ cells could be observed. Therefore, it was speculated, that the phenotype, reported by Trott and Morano (2004) and Spinazzola et al. (2006) might be specific to the strains, used in these studies and represent an indirect effect due to increased loss of mitochondrial DNA in these strains as reported (Spinazzola et al., 2006).

\subsubsection{Localization and topology of Sym1}

Initially, mouse MPV17 was reported to be localized in peroxisomes (Zwacka et al., 1994). However, more recent studies indicate a mitochondrial localization of human MPV17 (Spinazzola et al., 2006) as well as its yeast homolog Sym1 (Trott and Morano, 2004).

To verify this, a strain was generated that expresses GFP, fused to the C-terminus of Sym1 and the mitochondrial localization of Sym1 was confirmed by fluorescence microscopy (Fig 3.19A). Co-localization of MitoTracker Orange, a dye, specific for mitochondria, with Sym $1{ }^{\text {GFP }}$, indicated the mitochondrial localization of Sym1. 
Since Sym1 contains four predicted transmembrane spans, it was hypothesized, that it must be an integral membrane protein in mitochondria. To confirm this, isolated mitochondria were analyzed by carbonate treatment (Fig 3.19B). Aco1 (aconitase), a soluble matrix marker, and Tim44, which is peripherally associated to the inner membrane, could be extracted from the mitochondrial membranes by carbonate. In contrast, Sym1 and Por1, a polytopic outer membrane protein, were sedimented with the membrane fraction. Hence, it can be concluded that Sym1 is an integral membrane protein.

Furthermore, the submitochondrial localization and topology of Sym1 was addressed by protease protection assays of Sym1 (Fig 3.19C). Mitochondria from wild-type and a strain expressing ProteinA, fused to the C-terminus of Sym1, were incubated with increasing amounts of proteinase K. In contrast to Tom70, an outer membrane marker protein, Sym1 and Sym $1^{\text {Prot.A }}$ remained protected by the outer membrane. However, when mitoplasts were generated by osmotic swelling of the outer membrane, Sym1 and Sym1 Prot.A became accessible to protease. In SYMI Prot.A mitochondria, the protease accessibility of the Cterminal ProteinA tag revealed, that the C-terminus of Sym1 is exposed to the intermembrane space. Taking the four predicted transmembrane spans into consideration, it was concluded, that the N- and C- terminus as well as the central loop of Sym1 is exposed to the intermembrane space, whereas two loops are exposed to the mitochondrial matrix (Fig 3.19D). Interestingly this finding experimentally disproved the topology predicted by Wong et al. (2007) which suggested a matrix localization of the $\mathrm{N}$ - and C-terminus.

In wild-type mitochondria, Sym1 was detected using antiserum directed against the Cterminus (Fig 3.19C). Interestingly, the Sym1 C-terminus is more stable than the C-terminal ProteinA tag at lower protease concentrations. Most likely, the short C-terminal extension of Sym1 into the inter membrane space is not big enough to be accessible at lower protease concentrations. In addition, the C-terminus could be protected by interactions with proteins or membranes. 
A

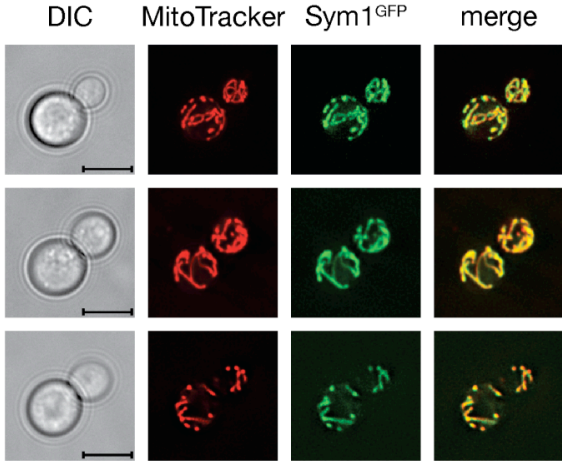

C

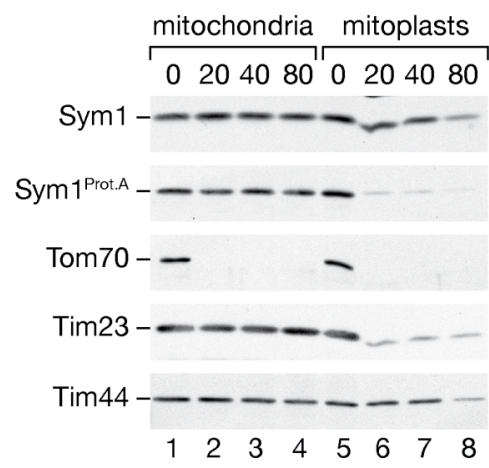

B

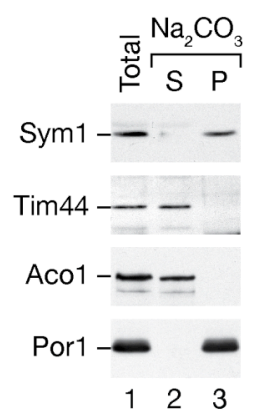

D

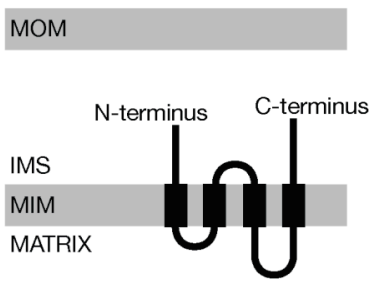

Figure 3.19 Sym1 is a protein of the mitochondrial inner membrane

(A) Cells, expressing Sym1 $1^{\text {GFP }}$ were visualized using a DeltaVision Deconvolution microscope in differential interference contrast (DIC). The mitochondrial network, stained with MitoTracker Orange (Invitrogen), and GFP signals were detected by fluorescence microscopy and merged to show colocalization. Bar, $5 \mu \mathrm{m}$. (B) Isolated wild-type mitochondria were incubated in $100 \mathrm{mM}$ sodium carbonate $(\mathrm{pH}$ 11.5). The soluble fraction (S) was separated from the membrane pellet (P) by centrifugation. $(\mathbf{C})$ Mitochondria and mitoplasts, generated by osmotic swelling, were incubated with increasing amounts of proteinase K (Prot. K), followed by SDS-PAGE and Western blotting. (D) Topology of Sym1. MOM, mitochondrial outer membrane; IMS, inter membrane space; MIM mitochondrial inner membrane.

\subsubsection{Formation and assembly of Sym1 complexes}

\subsubsection{Sym1 forms high molecular membrane complexes}

The peroxisomal homolog of Sym1/MPV17, Pmp22, was suggested to form multimeric complexes (Rokka et al., 2009). To address if Sym1 forms complexes in the mitochondrial inner membrane, mitochondria from cells, expressing Sym1, Sym1 ${ }^{\text {Prot.A }}$ or lacking Sym1 were solubilized in digitonin buffer and membrane protein complexes were separated by BNPAGE. Western blot detection of the ProteinA tag revealed two complexes of about 120 and $220 \mathrm{kDa}$ (Fig 3.20A). These complexes, named Sym $1_{\mathrm{c} 120}$ and Sym $1_{\mathrm{c} 220}$, could not be detected using antibody specific to the C-terminus of Sym1 (not shown), indicating that the Cterminus is buried inside of the complex and therefore not accessible for antibodies. 

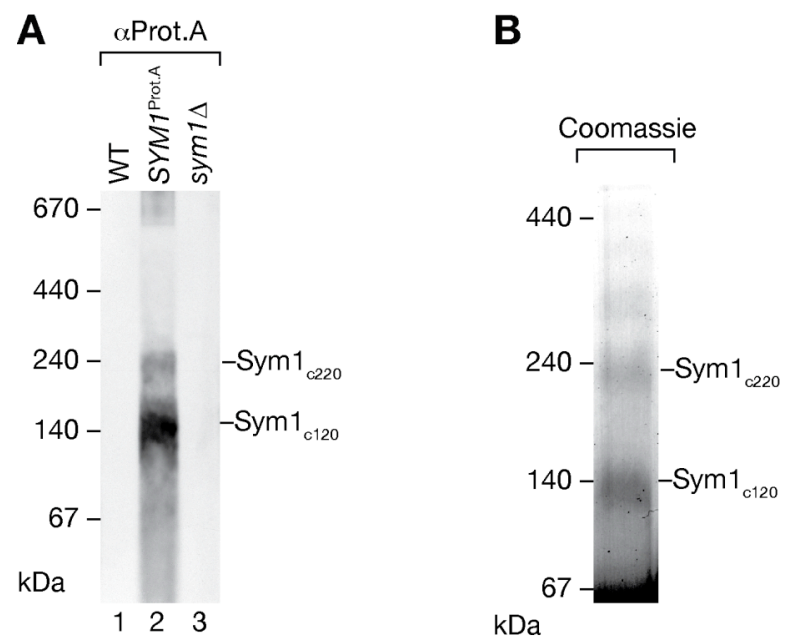

Figure 3.20 High molecular weight membrane complexes of Sym1

(A) Isolated mitochondria from indicated yeast stains were solubilized in 1\% digitonin and membrane complexes were analyzed by BN-PAGE and Western blotting. (B) Sym1 complexes were isolated by IgG chromatography from SYMI ${ }^{\text {Prot.A }}$ mitochondria. After elution by TEV-protease, complexes were subjected to BN-PAGE and Coomassie staining.

Additionally, these complexes were purified by IgG chromatography, eluted by TEVprotease cleavage under native conditions and could be Coomassie stained (Fig 3.20B). Hence, it could be shown, that Sym1 is present in high molecular weight complexes of about 120 and $220 \mathrm{kDa}$ in the mitochondrial inner membrane. Analysis of the composition and assembly of these complexes will help to understand the molecular function and biogenesis of Sym1.

\subsubsection{Establishment of an in vitro import assay of Sym1}

To analyze the mitochondrial assembly of the observed Sym1 complexes, an in vitro import/assembly assay of Sym1 was established. Therefore, $\left[{ }^{35} \mathrm{~S}\right]$ labeled Sym1 was synthesized in vitro using rabbit reticulocyte lysate. Subsequently, $\left[{ }^{35} \mathrm{~S}\right] \mathrm{Sym} 1$ was in vitro imported into isolated yeast mitochondria (Fig 3.21A). Protease protected Sym1 accumulated over time dependent on the membrane potential $(\Delta \psi)$. Interestingly, no processed form of Sym1 was detected. Therefore, in contrast to predictions by Spinazzola et al. (2006), Sym1 lacks a cleavable presequence. This was also validated by Edman degradation and mass spectrometric identification of the N-terminus of Sym1, purified from yeast mitochondria (not shown). 
A

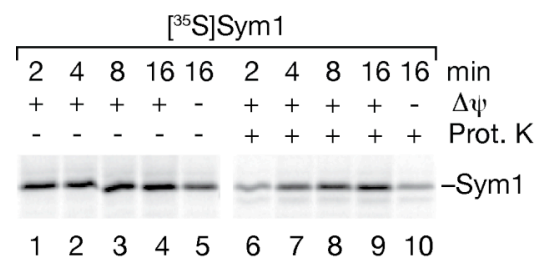

B

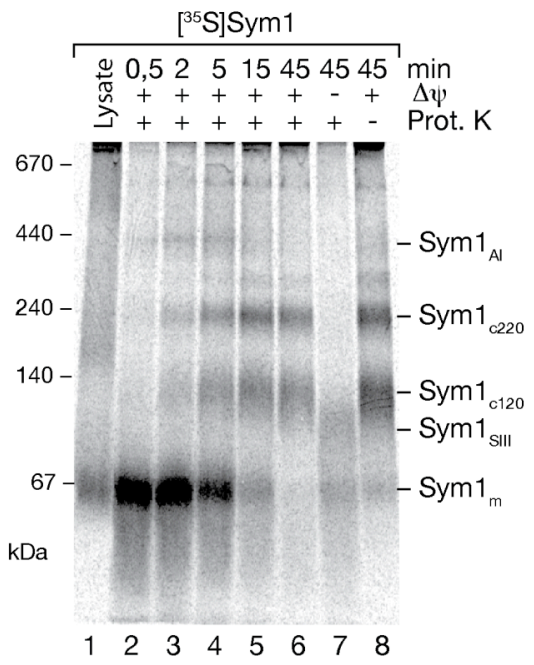

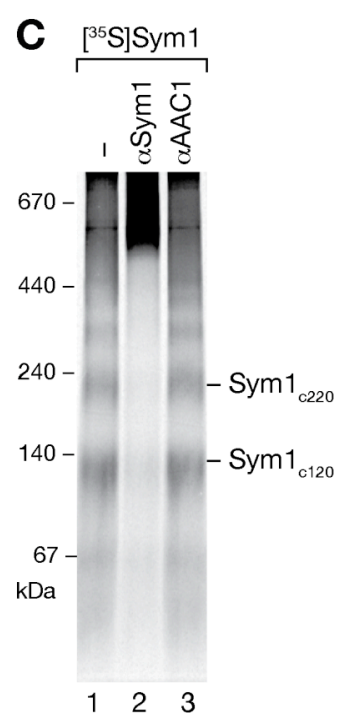

Figure 3.21 Assembly of Sym1 complexes

(A and B) $\left[{ }^{35} \mathrm{~S}\right] \mathrm{Sym} 1$ was in vitro imported into isolated yeast mitochondria for indicated time points in presence or absence of a membrane potential $(\Delta \psi)$ followed by proteinase K (Prot.K) treatment and SDSPAGE (A) or BN-PAGE (B) analysis. In (B) a lysate sample was used as a marker for monomeric Sym 1 $\left(\mathrm{Sym} 1_{\mathrm{m}}\right) . \mathrm{Sym} 1_{\mathrm{c} 120} / \mathrm{Sym} 1_{\mathrm{c} 220}$, Sym 1 complexes of about $120 / 220 \mathrm{kDa}$; Sym $1_{\mathrm{AI}}$, assembly intermediate; Sym1 $_{\text {SIII }}$, stage III intermediate. (C) After in vitro import of Sym1 and osmotic swelling of the outer mitochondrial membrane, indicated antibodies were added to the mitoplasts and samples were analyzed by BN-PAGE and digital autoradiography.

The assembly of the Sym $1_{\mathrm{c} 120 / \mathrm{c} 220}$ complexes were resolved in detail by BN-PAGE analysis of the newly assembled complexes after in vitro import (Fig 3.21B). After short incubation times and dependent on the membrane potential $(\Delta \psi)$, Syml accumulated at a protease protected location (Fig 3.21B lane 2 and 3). Because of the co-migration with the in vitro synthesized protein (lysate) at about $65 \mathrm{kDa}$, it was speculated that this band represents monomeric Sym1 $\left(\mathrm{Sym} 1_{\mathrm{m}}\right)$. Additionally, only after short incubation times, high molecular weight complexes of $420 \mathrm{kDa}$, most likely transient assembly intermediates (Sym1 $1_{\mathrm{AI}}$ ), were detected. After longer incubation (Fig 3.21B lanes 4 - 6), Sym1 assembled to the respective mature Sym $1_{\mathrm{c} 120}$ and $\mathrm{Sym} 1_{\mathrm{c} 220}$ complexes. Sym $1_{\mathrm{m}}$ and the assembly intermediate complexes $\left(\mathrm{Sym}_{\mathrm{AI}}\right)$ were not longer detected. Although there was no assembly to the $\mathrm{Sym} 1_{\mathrm{c} 120}$ and Sym $1_{\mathrm{c} 220}$ complexes without a membrane potential, minute amounts of $\mathrm{Sym} 1_{\mathrm{m}}$ and a complex of about $90 \mathrm{kDa}$ were detected (Fig 3.21B lane 7), which could represent a stage III import intermediate $\left(\mathrm{Sym}_{\mathrm{SIII}}\right)$ that accumulates during outer membrane translocation at the TOM in the absence of a membrane potential as also shown for AAC (Ryan et al., 1999).

Additionally, antibody shift experiments, revealed the specificity of the imported complexes (Fig 3.21C). 
Taken together, it could be shown, that Sym1 imports into mitochondria in a membrane potential dependent manner, and subsequently assembles into high molecular complexes.

\subsubsection{Sym1 assembly is not dependent on the TIM22-translocase}

Polytopic inner membrane proteins, that lack a cleavable presequence, including the metabolite carriers, are typically imported via the TIM22 translocase (Chacinska et al., 2009). Therefore it was speculated, that Sym1 is also a substrate of TIM22. To prove this hypothesis, the import and assembly was analyzed in mutant mitochondria, which have a temperature dependent defect of subunits of TIM22 (Gebert et al., 2008; Wagner et al., 2008).

In wild-type mitochondria, AAC, a well characterized TIM22 substrate, assembles into the mature dimer in the presence of a membrane potential (Fig. 3.22A lanes 9-12). However, mutations affecting Tim54 (Wagner et al., 2008), a core subunit of the TIM22 translocase lead to drastically reduced import and assembly of AAC, (Fig. 3.22A lanes 13-15). In contrast to that, the import and assembly of Sym1 was unaltered by mutations affecting Tim54 (Fig 3.22A lanes 1-8). This finding was verified in mitochondria isolated from tim12-4 cells, containing temperature sensitive Tim12, a peripheral subunit of TIM22 (Gebert et al., 2008): Although the assembly of AAC was impaired, Sym1 assembly was not reduced (Fig 3.22B).

Hence, Sym1 import is not affected by the mutations of the TIM22 translocase. Therefore, it was concluded that Sym1 is imported independent of TIM22 via an alternative import mechanism.

\subsubsection{Sym1 assembly is dependent on functional TIM23-translocase}

About $70 \%$ of the mitochondrial matrix/inner membrane proteins are substrates of TIM23 and contain an N-terminal presequence as a targeting signal (van der Laan et al., 2010; Vögtle et al., 2009). However, TIM23 also translocates proteins lacking an N-terminal presequence and containing internal targeting signals such as Bcs1 (Fölsch et al., 1996). Since it could be shown, that Sym1 is imported independent of TIM22, an import mechanism via TIM23 was considered. 

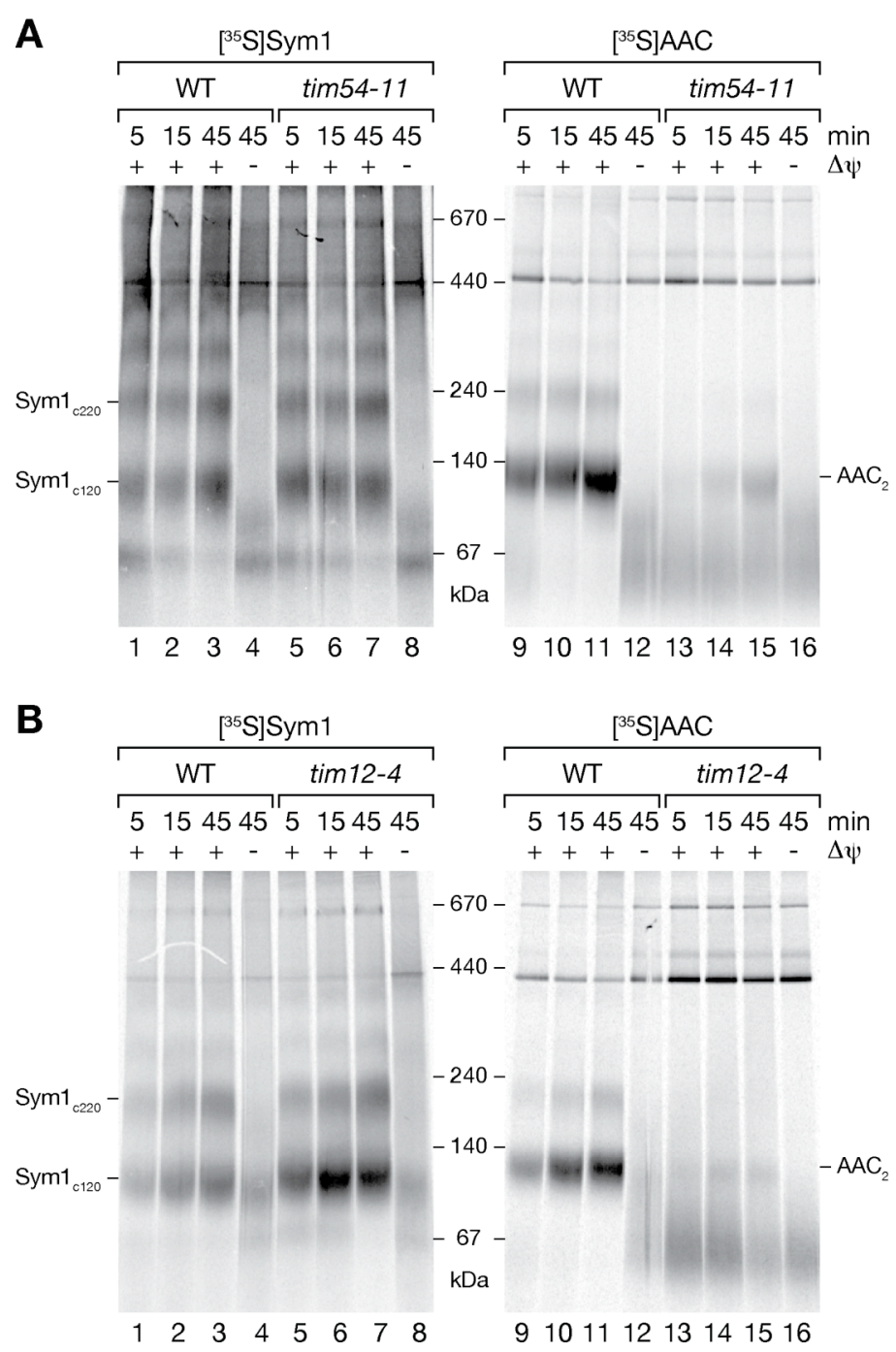

Figure 3.22 Sym1 assembly in TIM22 mutant mitochondria

After 15 min heat shock at $37{ }^{\circ} \mathrm{C}$ of wild-type and tim54-11 (A) or tim 12-4 (B) mitochondria, $\left[{ }^{35} \mathrm{~S}\right]$ labeled Sym1 or AAC was in vitro imported for indicated times in presence or absence of membrane potential $(\Delta \psi)$ prior to treatment with $40 \mu \mathrm{g} / \mathrm{ml}$ proteinase K. Sym1 complexes $\left(\mathrm{Sym}_{\mathrm{c} 120}, \mathrm{Sym} 1_{\mathrm{c} 220}\right)$ as well as $\mathrm{AAC}$ dimers $\left(\mathrm{AAC}_{2}\right)$ were detected after BN-PAGE and digital autoradiography.

To analyze if Sym1 import depends on TIM23, in vitro import/assembly assays were performed into mitochondria with defective TIM23. Surprisingly, Sym1 assembled less efficient and with delayed kinetics in tim50-1 mitochondria (Chacinska et al., 2005), which contain temperature sensitive Tim50, the receptor of TIM23 (Fig 3.23A). In contrast, AAC assembly was unaffected in these mitochondria compared to the wild-type. This finding was confirmed in tim23 $\downarrow$ mitochondria (Schulz et al., 2011), which were depleted of Tim23, the central subunit of TIM23 (Fig 3.23B). Although AAC import and assembly were comparable in wild-type and tim $23 \downarrow$ mitochondria, Sym1 assembly was strongly reduced when Tim23 

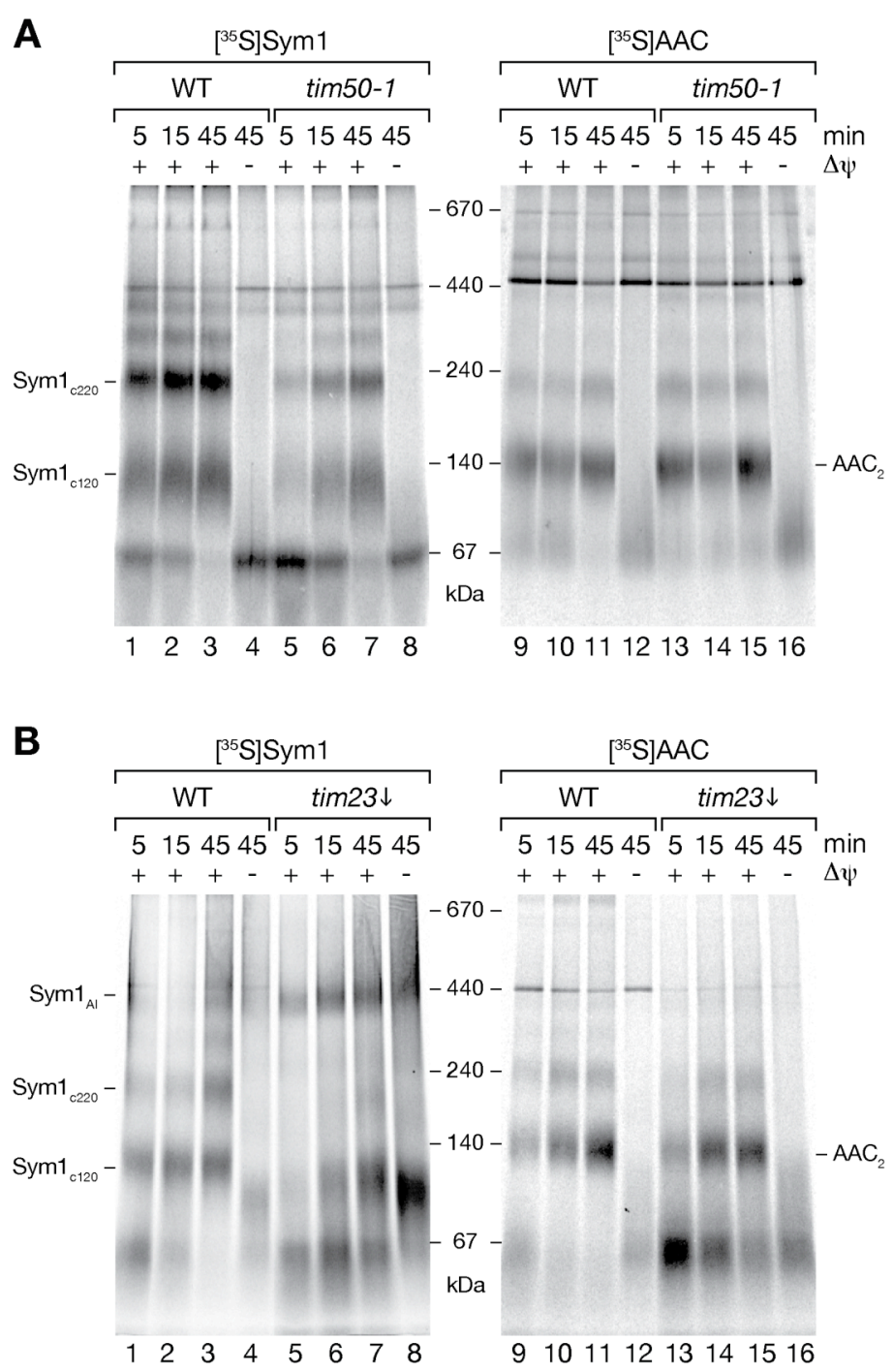

Figure 3.23 Sym1 assembly in TIM23 mutant mitochondria

Assembly analysis of $\left.{ }^{35} \mathrm{~S}\right] \mathrm{Sym} 1$ in wild-type and tim50-1 mitochondria after heat shock for $15 \mathrm{~min}$ at $37^{\circ} \mathrm{C}(\mathbf{A})$ or in wild-type and $\operatorname{tim} 23 \downarrow$ mitochondria (B). Experimental setup and conditions as described in (Fig 3.22). Complexes formed by Sym1 (Sym1 $\left.1_{\mathrm{c} 120}, \mathrm{Sym}_{1_{\mathrm{c} 220}}, \operatorname{Sym} 1_{\mathrm{AI}}\right)$ and $\mathrm{AAC}\left(\mathrm{AAC}_{2}\right)$ are indicated.

was depleted. Interestingly in tim $23 \downarrow$ mitochondria Sym1 accumulated in a complex of 420 $\mathrm{kDa}$, most likely the assembly intermediate $\left(\mathrm{Sym} 1_{\mathrm{AI}}\right)$, previously seen after short import times in wild-type mitochondria (Fig 3.21B). This import intermediate accumulated due to defective TIM23 translocase and most likely represents Sym1 bound to TOM.

Taken together, it was concluded that although Sym1 lacks a classical N-terminal presequence, its import and assembly depend on the TIM23 translocase. 
A
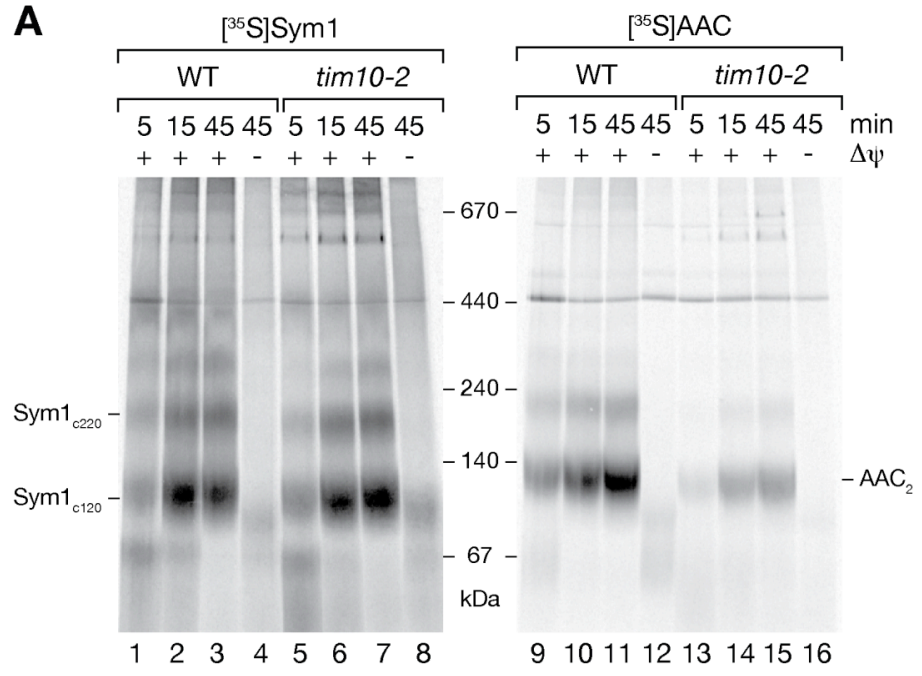

B

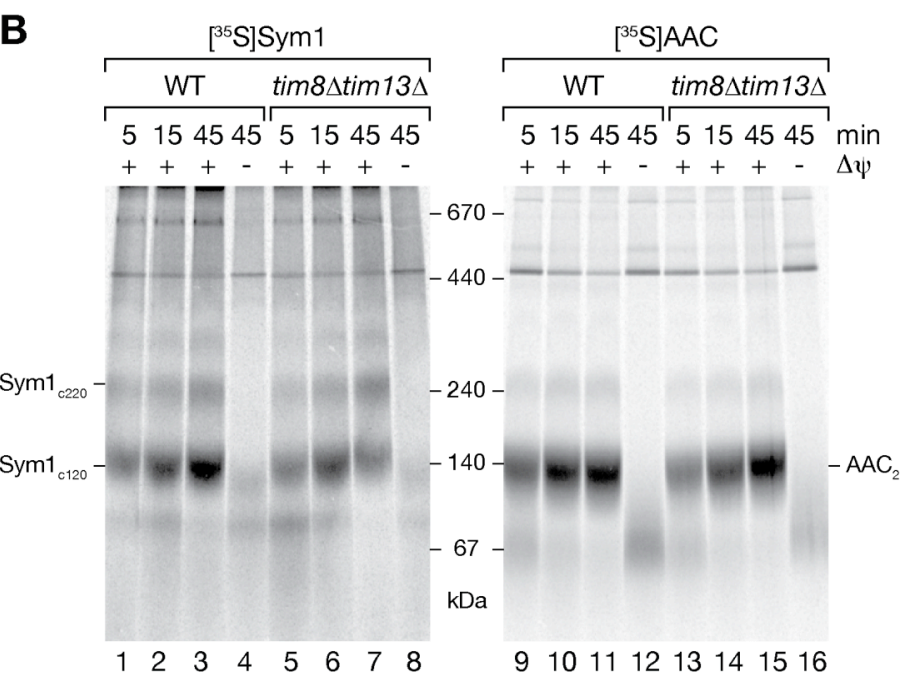

Figure 3.24 Sym1 assembly in small Tim mutant mitochondria

(A) Wild-type and tim 10-2 mitochondria were heat shocked at $37^{\circ} \mathrm{C}$ for $15 \mathrm{~min}$. Subsequently, in vitro import at $25^{\circ} \mathrm{C}$ for indicated times with or without a membrane potential $(\Delta \psi)$ and proteinase $\mathrm{K}$ $(40 \mu \mathrm{g} / \mathrm{ml})$ treatment was performed. Samples were subjected to BN-PAGE analysis and digital autoradiography. Sym $1_{\mathrm{c} 120} / \mathrm{Sym} 1_{\mathrm{c} 220}$, Sym 1 complexes; $\mathrm{AAC}_{2}$, mature AAC-dimer. (B) Same experimental setup as described in (A) with wild-type and $\operatorname{tim} 8 \Delta \operatorname{tim} 13 \Delta$ mitochondria, the heat shock was omitted.

\subsubsection{The influence of small Tim proteins on Sym1 assembly}

During mitochondrial import, so called small Tim proteins, shuttle hydrophobic cargo proteins from the outer membrane translocase to the inner membrane translocases (Petrakis et al., 2009). To address the influence of small Tim proteins on Sym1 biogenesis, in vitro import analysis was performed in mitochondria, that lack or have mutant small Tim proteins. Mitochondria, with temperature dependent defects of Tim10 (Truscott et al., 2002) showed 
A

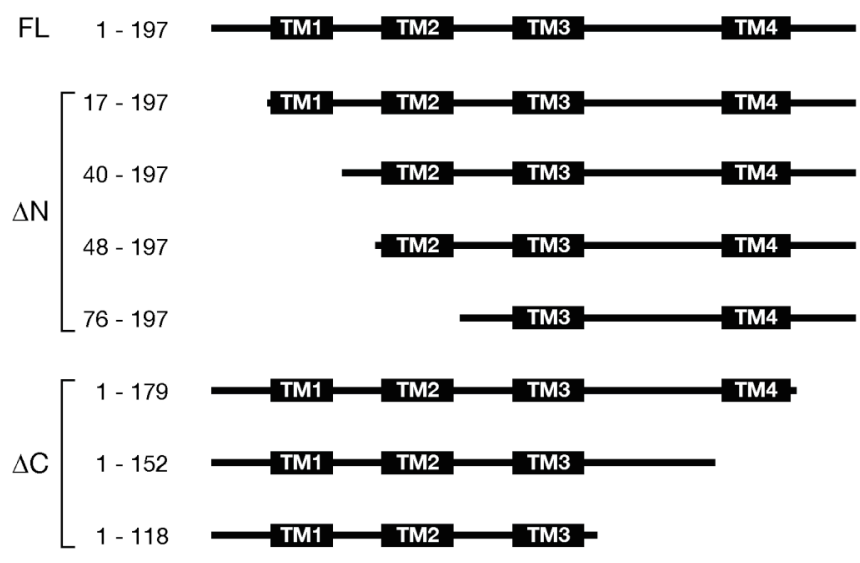

B $\quad \mathrm{FL} \quad \Delta \mathrm{N} \quad \Delta C$

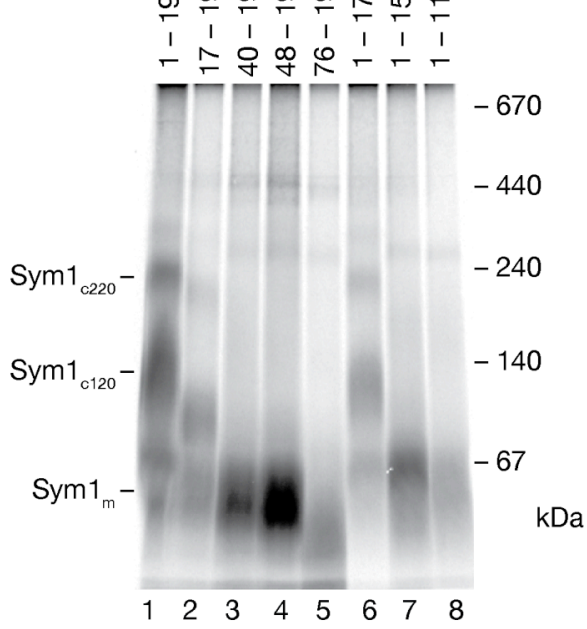

Figure 3.25 Assembly of truncated Sym1 proteins

(A) Schematic representation of Sym1 truncation constructs, used for in vitro import analyses. FL, full length; $\Delta \mathrm{N}$, N-terminal truncation; $\Delta \mathrm{C}, \mathrm{C}$-terminal truncation; $\mathrm{TM}$, transmembrane span. (B) In vitro import of $\left[{ }^{35} \mathrm{~S}\right]$ Sym 1 truncation-constructs as depicted in (A). Samples were treated with $40 \mu \mathrm{g} / \mathrm{ml}$ proteinase $\mathrm{K}$ and analyzed by BN-PAGE and digital autoradiography. Sym1 complexes $\left(\operatorname{Sym} 1_{\mathrm{c} 120}\right.$, $\left.\mathrm{Sym}_{\mathrm{c} 220}\right)$ and monomeric Sym1 $\left(\mathrm{Sym} 1_{\mathrm{m}}\right)$ are indicated for the full length construct.

strongly reduced AAC assembly, whereas the assembly of Sym1 was unaffected, compared to wild-type mitochondria (Fig 3.24A). Moreover, mitochondria lacking Tim8 and Tim13, small Tim proteins involved in the import of Tim23 (Leuenberger et al., 1999; Truscott et al., 2002) did not show any import defects for AAC. Sym1 also assembled into the mature

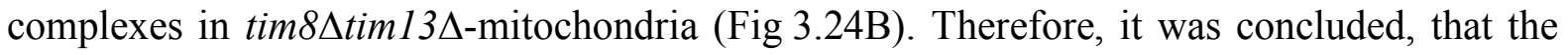
small Tim proteins Tim8, Tim10 and Tim13 are not essential for Sym1 import and assembly. However, the influence of so far uncharacterized inter membrane space chaperones cannot be excluded.

\subsubsection{Mapping Sym1 domains, necessary for its import and assembly}

Sym1 does not contain a cleavable N-terminal presequence that targets the protein to mitochondria (see section 3.2.2.2). To map mitochondrial targeting signals of Sym1, truncation constructs of Sym1 were in vitro synthesized and $\left[{ }^{35} \mathrm{~S}\right]$ labeled (Fig 3.25A). Subsequently, the import competence of these constructs was analyzed by in vitro import and assembly into isolated mitochondria. Interestingly, the constructs lacking the $\mathrm{N}$ - or Cterminal inter membrane space domains were still imported in mitochondria and even assembled into high molecular weight complexes (Fig 3.25B lanes 2 and 6). However, these 
complexes appear smaller in size, compared to the complexes of full length Sym1 (Fig 3.25B lane 1). This size shift could be due to the truncation of Sym1 or lost protein interactions.

More extensive $\mathrm{N}$-terminal truncations, additionally removing the first transmembrane span and the first matrix loop, lead to assembly defects of Sym1 (Fig 3.25B lanes 3 and 4). The import of these constructs however, was not affected since monomeric Sym1 (Sym1 $1_{\mathrm{m}}$ ) still accumulated. In contrast, a Sym1 truncation, also lacking the second transmembrane span, showed only minor import efficiency (Fig 3.25B lane 5).

Similar to these findings, C-terminal truncations of Sym1 revealed, that the 4th transmembrane span is required for assembly but not for import (Fig 3.25B lane 7), whereas further truncation of the second matrix loop drastically reduced import efficiency.

Hence, it can be concluded that mitochondrial targeting signals are distributed at different sites of Sym1. Furthermore, it could be shown, that the inter membrane space termini are dispensable, the terminal transmembrane spans however, are required for assembly of Sym1.

\subsubsection{Modeling MPV17 patient mutations using SYM1}

\subsubsection{Mutant Sym1 proteins are stable in mitochondria}

The involvement of MPV17 in the Mitochondrial DNA Depletion Syndrome was first shown by identification of patient missense mutations leading to $\mathrm{R}^{50} \mathrm{Q}$ or $\mathrm{N}^{166} \mathrm{~K}$ exchanges in MPV17 (Spinazzola et al., 2006).

To study the effect of these point mutations, corresponding mutations in SYM1 were used as a model. Although no respiratory phenotype in sym $1 \Delta$ cells could be observed (Fig 3.18A), a deleterious effect of the mutant Sym1 variants could not be excluded. Therefore, in this study these variants of Sym1 were expressed from a plasmid in sym $1 \Delta$ cells and growth tests were performed. In contrast to previous studies (Spinazzola et al., 2006), these cells did not show growth defects on ethanol containing media at heat shock conditions (Fig 3.26A).

However it was speculated, that mutations could lead to rapid turnover of $\mathrm{Sym} 1^{\mathrm{R} 51 \mathrm{Q}}$ or Sym $1^{\mathrm{N} 172 \mathrm{~K}}$, as previously speculated for MPV $17^{\mathrm{R} 50 \mathrm{Q}}$ (Spinazzola et al., 2006) and shown for Shy $1^{\mathrm{G} 137 \mathrm{E}}$ and Shy $1^{\mathrm{G} 137 \mathrm{R}}$ in this study. To analyze this hypothesis, mitochondria from cells, expressing the wild-type or mutant variants of Sym1 were isolated and Sym1 levels were compared (Fig 3.26B). It should be noted, that the mutations, analyzed in this study, do not alter the recognition site of the Sym1-specific antibody, as it is directed against the C- 
A

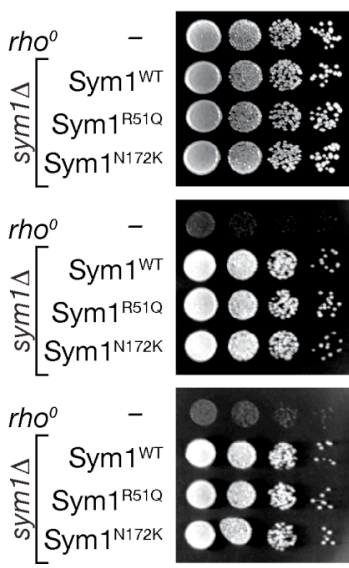

$30^{\circ} \mathrm{C}$
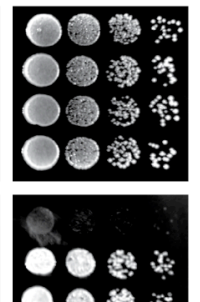

$35^{\circ} \mathrm{C}$
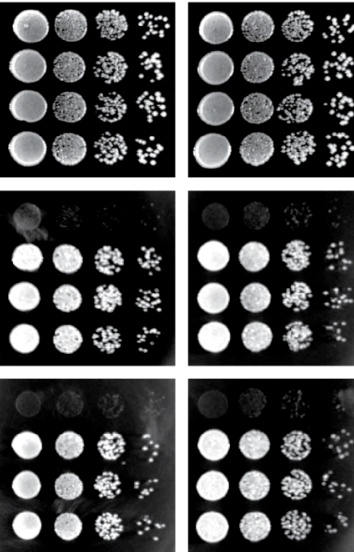

B

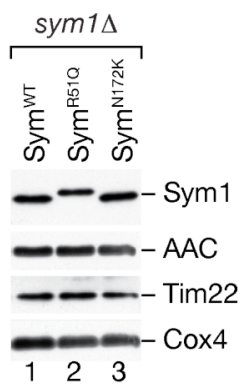

Figure 3.26 Mimicking patient mutations in yeast Sym1

(A) Growth test of cells, lacking mitochondrial DNA $\left(r h o^{\circ}\right)$ and containing an empty vector, or sym $1 \Delta$ cells expressing Sym $1^{\mathrm{WT}}$, Sym $1^{\mathrm{R} 51 \mathrm{Q}}$ or $\mathrm{Sym} 1^{\mathrm{N} 172 \mathrm{~K}}$ from a plasmid. Serial dilutions of cells were spotted on selective media plates, containing glucose (SD-ura), glycerol (SG-ura) or ethanol (SE-ura) as a carbon source and incubated at indicated temperatures. (B) $15 \mu \mathrm{g}$ isolated mitochondria from sym $1 \Delta$ cells, expressing $\mathrm{Sym} 1^{\mathrm{WT}}, \mathrm{Sym}^{\mathrm{R} 51 \mathrm{Q}}$ or $\mathrm{Sym} 1^{\mathrm{N} 172 \mathrm{~K}}$ from a vector, were analyzed by SDS-PAGE and Western blotting, using the indicated antibodies.

terminus. Interestingly, the abundance of the mutant Sym1 variants was indistinguishable from Sym $1^{\mathrm{WT}}$. Hence, the mutant forms of Sym1 were stable after mitochondrial import.

\subsubsection{Sym $1^{\mathrm{R} 51 \mathrm{Q}}$ and $\mathrm{Sym} 1^{\mathrm{N} 172 \mathrm{~K}}$ assembles into high molecular complexes}

Since the stability of Sym1 $1^{\mathrm{R} 51 \mathrm{Q}}$ and $\mathrm{Sym} 1^{\mathrm{N} 172 \mathrm{~K}}$ was not affected by the mutations, it was asked if these proteins form high molecular complexes or if complex formation is impaired due to the amino acid exchanges.

Therefore, membrane complexes of $s y m 1^{R 51 Q}$, sym $1^{N 172 K}$ and wild-type mitochondria were analyzed by BN-PAGE and Western blot analysis. Since the Sym1 specific antibody did not recognize complexes when the $\mathrm{BN}$-gel was directly analyzed by Western blotting (see section 3.2.2.1), subunits were separated by a second dimension SDS-PAGE prior to Western blot analysis (Fig 3.27). Interestingly, the mutant variants of Syml could be detected in high molecular weight complexes of identical size as Sym1 ${ }^{\mathrm{WT}}$. This indicates that complex formation and protein interactions are not impaired by the mutations in SYM1 affecting $\mathrm{R}^{51}$ and $\mathrm{N}^{172}$. 


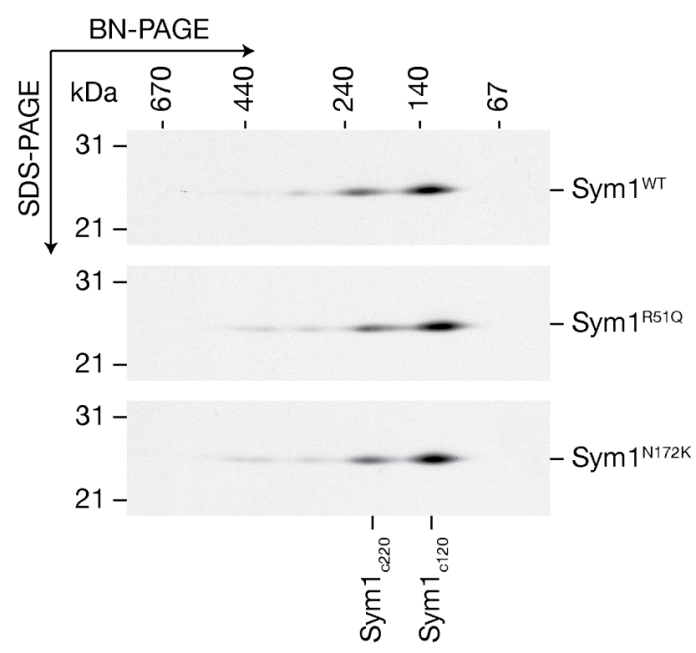

Figure 3.27 Complex formation of mutant Sym1 proteins

Mitochondria, from sym1 $1 \Delta$ cells, expressing the indicated Sym1 variants were solubilized in $1 \%$ digitonin. Proteins were separated by 2-dimensional BN-PAGE/SDS-PAGE and analyzed by Western blotting, using antibodies specific to the C-terminus of Sym1.

\subsubsection{Assembly of mutant Sym1 proteins}

Mutations affecting Sym1 did not impair the assembly of Sym1 into the respective mature complexes $\left(\mathrm{Sym}_{\mathrm{c} 120 / \mathrm{c} 220}\right)$. The kinetics of complex-assembly of $\mathrm{Sym} 1^{\mathrm{R} 51 \mathrm{Q}}$ and $\mathrm{Sym} 1^{\mathrm{N} 172 \mathrm{~K}}$ was analyzed by in vitro import analysis of $\left[{ }^{35} \mathrm{~S}\right]$ labeled $\mathrm{Sym} 1^{\mathrm{R} 51 \mathrm{Q}}$ and $\mathrm{Sym} 1^{\mathrm{N} 172 \mathrm{~K}}$ in comparison to the wild-type protein. It could be shown that the mutant variants assembled in a comparable manner as Sym $1^{\mathrm{WT}}$ (Fig 3.28). Hence, complex formation and assembly kinetics are unaffected by an $\mathrm{R}^{51} \mathrm{Q}$ or $\mathrm{N}^{172} \mathrm{~K}$ exchange in Sym1.

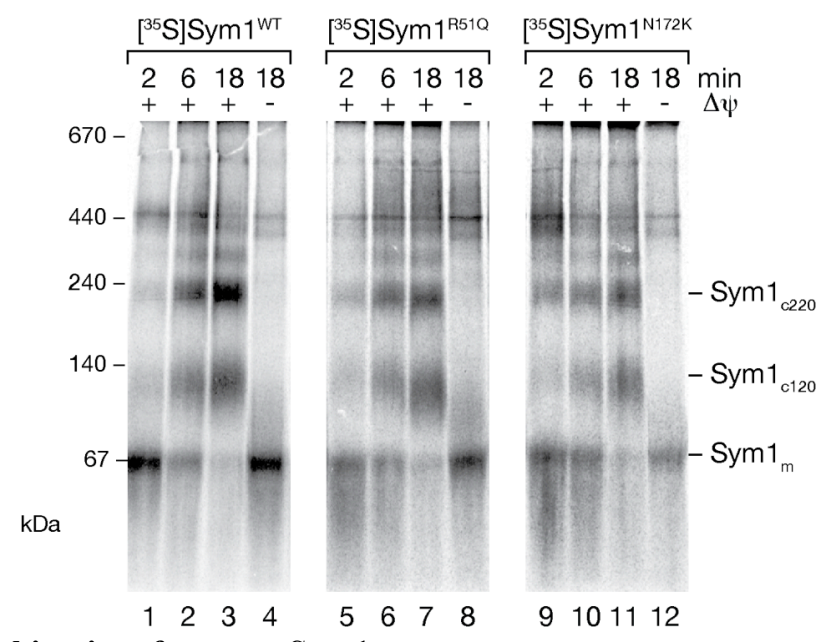

Figure 3.28 Assembly kinetics of mutant Sym1

(A) $\left[{ }^{35}\right.$ S $]$ labeled Sym $1^{\text {WT }}, \operatorname{Sym} 1^{\mathrm{R} 51 \mathrm{Q}}$ or Sym1 ${ }^{\mathrm{N} 172 \mathrm{~K}}$ was in vitro imported into wild-type mitochondria as indicated. Monomeric Sym1 $\left(\mathrm{Sym}_{\mathrm{m}}\right)$ and Sym1 complexes $\left(\mathrm{Sym} 1_{\mathrm{c} 120} / \mathrm{Sym} 1_{\mathrm{c} 220}\right)$ were detected after BNPAGE and digital autoradiography. $\Delta \psi$, membrane potential. 


\subsubsection{Sym1 forms a pore in the mitochondrial inner membrane}

\subsubsection{Purification of Sym1 from yeast mitochondria}

To date there is no experimental data on the molecular function of Sym1/MPV17. However, it was speculated previously that PMP22, the peroxisomal homolog of Sym1/MPV17, could form a nonselective pore in the peroxisomal membrane (Van Veldhoven et al., 1987). This was recently verified by electrophysiological characterization of mouse PMP22 (Rokka et al., 2009). Therefore, it was hypothesized that Sym1 could form a pore in the mitochondrial inner membrane. To analyze this, Sym1 was purified from yeast mitochondria for subsequent electrophysiological characterization, in this study.

A combination of $\mathrm{Ni}^{2+}$-affinity chromatography, IgG-chromatography and subsequent specific elution by TEV-protease cleavage of the ProteinA tag was established to purify Sym1 from mitochondria of cells expressing Sym1 ${ }^{\text {Prot.A }}$ (Fig 3.29A and B). As a control for contaminations, the purification was performed in wild-type mitochondria in parallel.

To validate the purification, the final elution fraction was analyzed by SDS-PAGE and subsequent Coomassie staining. Only one band was present, indicating the purity of the isolation (Fig 3.29C). Additionally, the identity of the purified Sym1 was verified by mass spectrometry (not shown).

A

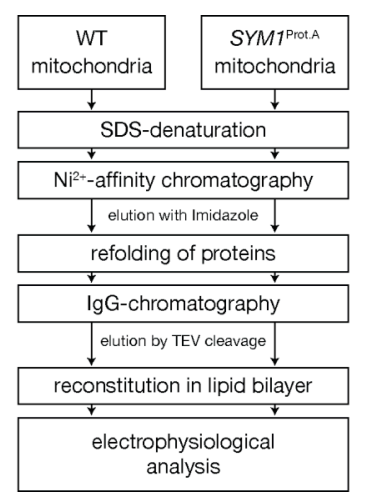

B

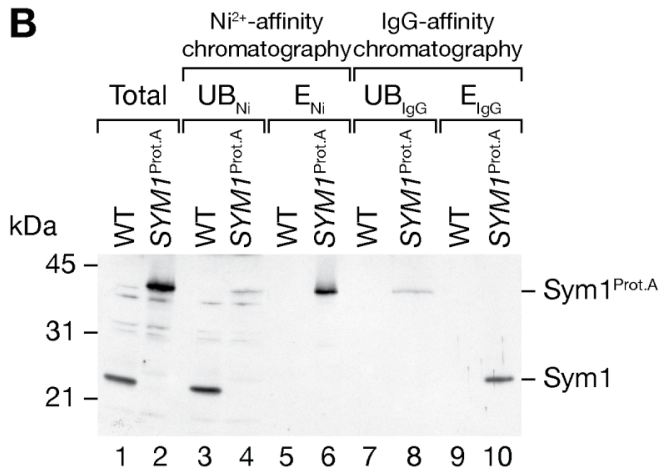

C

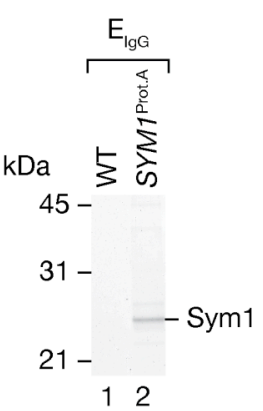

Figure 3.29 Purification of Sym1 for electrophysiological characterization

(A) Scheme of the purification strategy for subsequent electrophysiology. (B) Samples of the purification, as depicted in (A) were separated by SDS-PAGE and analyzed by Western blotting. UB, unbound; E, eluate. (C) The eluate of the IgG chromatography was analyzed by SDS-PAGE and Coomassie staining to assess purity of the preparation. 


\subsubsection{Electrophysiological characterization of Sym1}

Sym1, purified from yeast mitochondria, was electrophysiologically characterized in collaboration with Dr. M. Meinecke and Dr. V. Krüger (AG Wagner, Universität Osnabrück). Purified Sym1 was incorporated into a planar lipid bilayer and current recordings were performed (Fig 3.30). Remarkably a pore forming activity with a main conductance state of $450 \mathrm{pS}$ at $250 \mathrm{mM}$ potassium chloride, $20 \mathrm{mM}$ MOPS/Tris $\mathrm{pH} 7.0$ could be detected. This was independent of the polarization of the applied holding potential. The conductance state corresponds to a pore size of about $1.6 \mathrm{~nm}$. Interestingly, voltage increase led to transient reduction of conductance. These gating events of the channel indicate a dynamic activity of the pore at high membrane potentials. It should also be noted that the eluate from the purification of the strain without a tagged protein (wild-type strain) was used as a negative control and showed no channel activity.

Moreover, a reverse potential of $48 \mathrm{mV}$ was determined (asymmetrical buffer conditions: $20 \mathrm{mM}$ potassium chloride, $20 \mathrm{mM}$ MOPS/Tris, $\mathrm{pH} 7.0$ at trans-side and $250 \mathrm{mM}$ potassium chloride, $20 \mathrm{mM} \mathrm{MOPS/Tris,} \mathrm{pH} 7.0$ at cis-side), indicating selectivity for cations of the channel (Fig 3.31A). Furthermore, the specificity of the channel activity was verified by addition of Sym1 specific antibodies, which reduced the conductance of the channel (Fig 3.31B).

Taken together, it can be concluded, that Sym1 forms a channel in the mitochondrial inner membrane, which favors cations and shows dynamic gating behavior. 

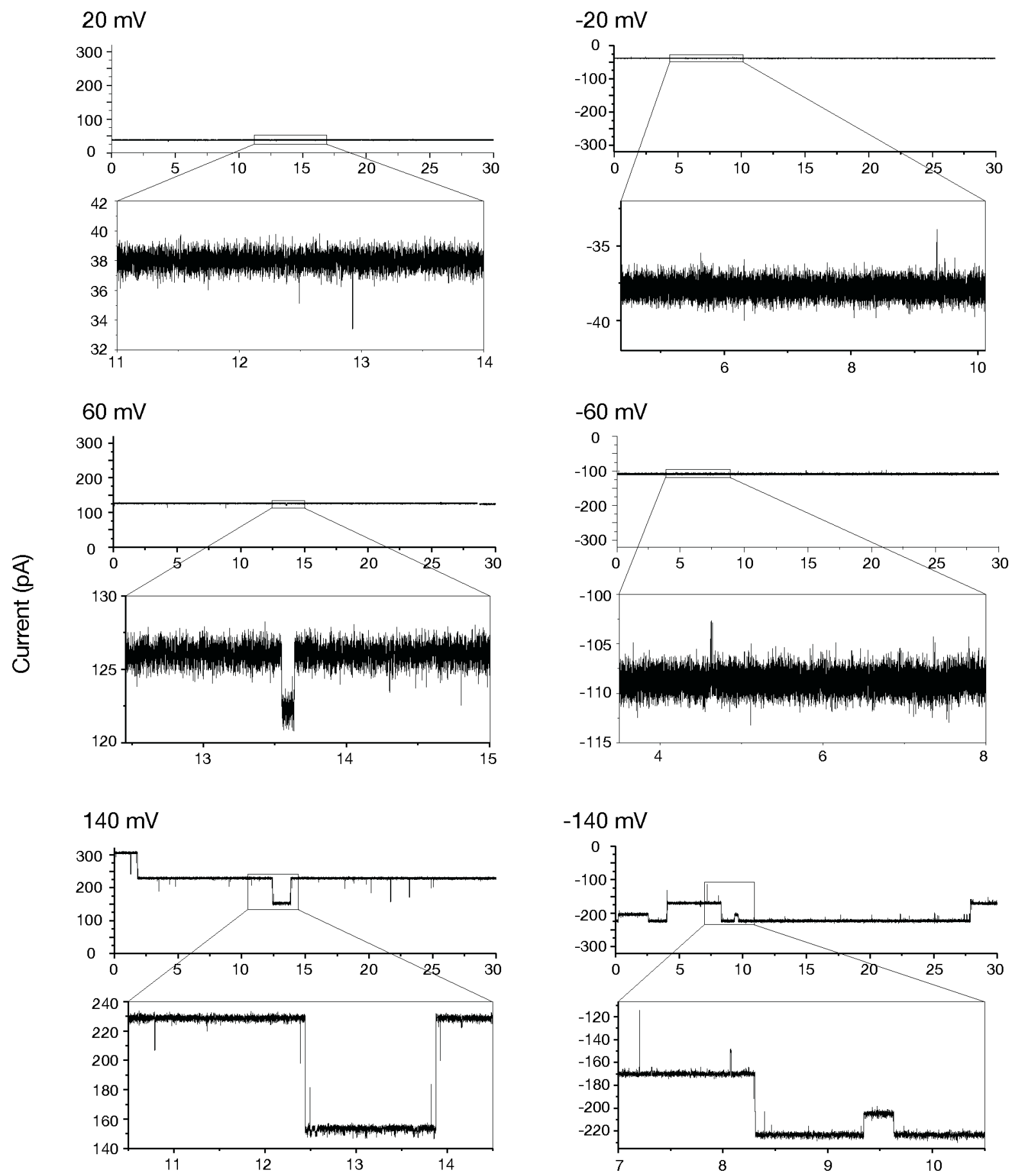

Time (s)

Figure 3.30 Sym1 shows channel activity

Sym1 was reconstituted in liposomes and incorporated in a planar lipid bilayer. Subsequently, current recordings at different voltages and polarizations of the bilayer (as indicated) were performed using $250 \mathrm{mM}$ potassium chloride, $20 \mathrm{mM}$ MOPS/Tris $\mathrm{pH} 7.0$ at both sides of the bilayer. 
A

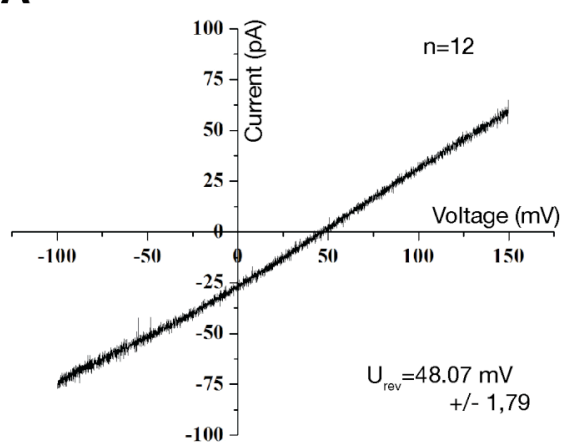

B

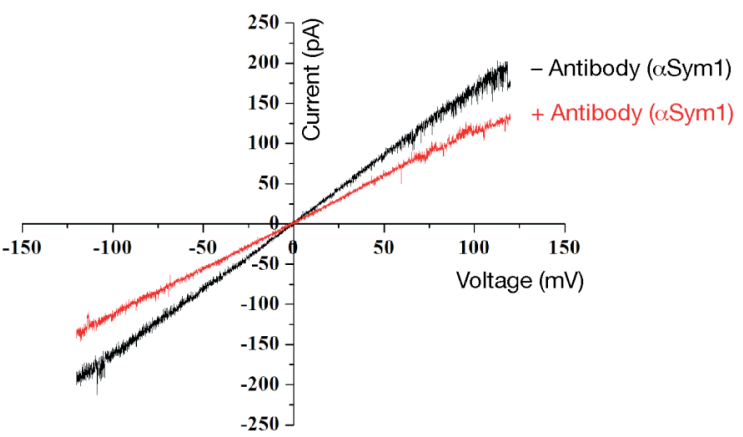

Figure 3.31 Cation selectivity and specificity of the Sym1-pore

(A) Sym1 was incorporated in a planar lipid bilayer. Using an asymmetric buffer system (trans-side: $20 \mathrm{mM}$ potassium chloride, $20 \mathrm{mM}$ MOPS/Tris, $\mathrm{pH} 7.0$; cis-side: $250 \mathrm{mM}$ potassium chloride, $20 \mathrm{mM}$ MOPS/Tris, $\mathrm{pH} \mathrm{7.0),} \mathrm{the} \mathrm{current/voltage} \mathrm{relationship} \mathrm{was} \mathrm{recorded} \mathrm{to} \mathrm{determine} \mathrm{the} \mathrm{reverse} \mathrm{potential}$ $\left(\mathrm{U}_{\text {rev }}\right)$ of the Sym1-pore. (B) Current/voltage relationship of Sym1 before and after addition of Sym1 specific antibodies at symmetric buffer conditions $(250 \mathrm{mM}$ potassium chloride, $20 \mathrm{mM}$ MOPS/Tris $\mathrm{pH}$ $7.0)$. 


\section{Discussion}

In this study, the yeast $S$. cerevisiae was used as a model to unravel the molecular pathology of certain patient point mutations, affecting nuclear genes that encode mitochondrial proteins. Although a lot is known about the molecular pathology of mitochondrial DNA mutations, nuclear gene mutations leading to mitochondrial dysfunction are less understood (Finsterer, 2004). Often, the molecular function of proteins, involved in mitochondrial diseases, is still unclear and functional characterization of these proteins will also help to shed light in their dysfunction.

SURF1 and MPV17 are proteins of ill-defined function involved in the development of severe mitochondrial diseases (see section 1.4). In this study, the molecular pathology of three point mutations in SURF1, identified in Leigh Syndrome patients was analyzed. Furthermore, the molecular function and biogenesis of Sym1, the yeast homolog of MPV17, which is involved in the Mitochondrial DNA Depletion Syndrome, was characterized.

\subsection{Molecular pathology of SURF1 point mutations}

Mutations in SURF1 are the main cause of Leigh Syndrome with isolated cytochrome $c$ oxidase deficiency. Although most patients have deletions or nonsense mutations in SURF1 (Pequignot et al., 2001), few missense mutations were described. Mutations affecting the amino acids $\mathrm{G}^{124}$ and $\mathrm{Y}^{274}$ of SURF1 were the first missense mutations identified in patients (Coenen et al., 1999; Poyau et al., 2000; Teraoka et al., 1999). Interestingly, these amino acids are highly conserved, which indicates their functional importance. Therefore, analysis of these mutations will not only help to understand the pathogenesis of the Leigh Syndrome, it will also improve our knowledge of the molecular function of SURF1 in the assembly process of the cytochrome $c$ oxidase.

As models for the $S U R F 1^{G 124 E}, S U R F 1^{G 124 R}$ and SURF1 $1^{Y 274 D}$ mutations, the corresponding yeast mutations in SHY1 (shyl $1^{G 137 E}$, shyl $1^{G 137 R}$, shyl $1^{Y 344 D}$ ) were analyzed in this study. Previous studies (Bestwick et al., 2010a) could only identify respiratory defects in shyl ${ }^{\text {Gl37E }}$ cells, whereas shy $1^{Y 344 D}$ cells showed no respiratory deficiency. Here, using a more extensive screening method for respiratory growth, it could be shown, that shyl ${ }^{Y 344 D}$ cells have a cold sensitive respiratory defect. Furthermore, the phenotype of $s h y l^{G 137 E}$ cells was verified and the respiratory deficiency of $s h y 1^{G 137 R}$ cells was shown. Due to the respiratory phenotype, 
these mutations can be well studied in yeast. Interestingly, the different temperature dependency of the phenotypes may already be a hint for different natures of protein dysfunction.

Different modes of molecular pathology due to mutations in nuclear-encoded mitochondrial proteins are feasible: First, point mutations can result in nonfunctional proteins as reported for the mitochondrial DNA polymerase (Ponamarev et al., 2002). In this case a $\mathrm{Y}^{955} \mathrm{C}$ exchange in the active center of pol $\gamma$ leads to defective DNA synthesis and disease. Furthermore point mutations can lead to mistargeting of the protein, as reported for the $\mathrm{V}^{223} \mathrm{D}, \mathrm{V}^{224} \mathrm{R}$ and $\mathrm{I}^{226} \mathrm{P}$ mutations in tafazzin (Claypool et al., 2006), a protein involved in Barth Syndrome (Barth et al., 2004). Another example for mistargeting is the $\mathrm{R}^{10} \mathrm{P}$ exchange in the presequence of the $\mathrm{PDH} \mathrm{E} 1 \alpha$ precursor, identified in patients with pyruvate dehydrogenase deficiency (Takakubo et al., 1995).

Using in vitro import and protease protection analysis of Shy1 and SURF1 precursors, it could be shown that the mitochondrial import and maturation is not affected by the mutations analyzed in this study. The reduced abundance of Shy1 with mutations in $\mathrm{G}^{137}$, observed in this study and reported previously (Bestwick et al., 2010a) can therefore not be explained by an impaired mitochondrial import efficiency. However, it could be shown that in contrast to Shy1 ${ }^{\text {Y344D }}$ (SURF1 ${ }^{\text {Y274D }}$ in human), which remained stable after import, mutations affecting $\mathrm{G}^{137}$ in Shy1 $\left(\mathrm{G}^{124}\right.$ in SURF1) lead to rapid degradation of the protein after mitochondrial translocation. This finding also explains the shy $1^{G 137 E}$ phenotype, which resembles a deletion of SHY1 in respect to respiratory deficiency and heme insertion in Cox1 (Bestwick et al., 2010a). Moreover, it explains the pathogenesis of patients with $S U R F 1^{G 124 E / R}$ mutations, which is similar to deletions or nonsense mutations of SURF1 (Coenen et al., 1999; Poyau et al., 2000).

In contrast, the mutation affecting $\mathrm{Y}^{344}$ of Shy1 ( $\mathrm{Y}^{274}$ of SURF1) did not reduce stability of the encoded protein after mitochondrial import. Additionally it was shown that the membrane topology of Shyl was not affected by an $\mathrm{Y}^{344} \mathrm{D}$ exchange. Therefore, altered mitochondrial translocation, maturation, topology or stability of Shy1 ${ }^{\mathrm{Y} 344 \mathrm{D}} / \mathrm{SURF} 1^{\mathrm{Y} 274 \mathrm{D}}$ does not account for the respiratory phenotype of the yeast cells and the development of Leigh Syndrome in affected patients. Instead, impaired function of Shy $1^{\text {Y344D } / S U R F 1 ~}{ }^{\text {Y274D }}$ could be shown.

The function of Shy1/SURF1 in the assembly process of the cytochrome $c$ oxidase remains still enigmatic. Yeast mitochondria lacking Shyl show an accumulation of cytochrome $c$ 
oxidase assembly intermediates, containing Cox1, Coa1, Coa3 and Cox14 (Barrientos et al., 2002; Mick et al., 2010; Mick et al., 2007; Pierrel et al., 2007). These assembly intermediates recruit Mss51, the translational activator of Cox1 and thereby shut down further Cox1 expression. The negative feed back regulation of Cox1 translation prevents accumulation of pro-oxidant assembly intermediates of the cytochrome $c$ oxidase in the mitochondrial inner membrane that are believed to generate reactive oxygen species (Khalimonchuk et al., 2007). Interestingly, a $\mathrm{Y}^{344} \mathrm{D}$ exchange in Shy1 does not shut down Cox1 expression. Furthermore, compared to shyld, in shyl $1^{Y 344 D}$ mitochondria Cox1 does not accumulate in Mss51 containing early assembly intermediates of the cytochrome $c$ oxidase. Moreover, analyses by Bestwick et al. (2010a) indicate that Shy $1^{\mathrm{Y} 344 \mathrm{D}}$ allows heme $a$ insertion in Cox1 which occurs downstream of negative feedback regulation of Cox 1 expression (Khalimonchuk et al., 2010; Khalimonchuk et al., 2007). Nevertheless, it was shown that in shyl ${ }^{Y 344 D}$ mitochondria less mature cytochrome $c$ oxidase can assemble. In fact, at non-permissive temperature, mature cytochrome $c$ oxidase is not detectable in shyl $1^{Y 344 D}$ mitochondria. Furthermore, when overexpressed in wild-type background, Shy $1^{\mathrm{Y} 344 \mathrm{D}}$ shows a dominant negative effect on Cox1 stability. This indicates that Shy $1^{\mathrm{Y} 344 \mathrm{D}}$ has a deleterious effect and generates a block of cytochrome $c$ oxidase assembly.

Taken together, in shy $1^{Y 344 D}$ mitochondria, Cox 1 progresses through early assembly steps with translational feed back regulation, subsequently heme $a$ is inserted but further assembly to mature cytochrome $c$ oxidase is blocked due to the $\mathrm{Y}^{344} \mathrm{D}$ exchange in Shy 1 and Cox 1 is degraded by the mitochondrial quality control system. Therefore it has to be concluded that Shy $1^{\text {Y344D }}$ (or SURF1 ${ }^{\mathrm{Y} 274 \mathrm{D}}$ ) is a partially nonfunctional protein (also see section 4.4), which, in respect to pathogenesis and phenotype of this mutation, is clearly different from mutations affecting $G^{137}$ of Shy1 ( $\mathrm{G}^{124}$ of SURF1).

In comparison, mutations affecting $\mathrm{G}^{137}$ of Shyl ( $\mathrm{G}^{124}$ of SURF1) lead to degradation whereas mutations affecting $\mathrm{Y}^{344}$ of Shy1 ( $\mathrm{Y}^{274}$ of SURF1) lead to impaired function of the protein. Therefore the phenotype or molecular pathology of the point mutations in SHY1 or $S U R F 1$, analyzed in this study, is caused by different mechanisms. As a consequence also differences in potential upcoming therapies for affected patients have to be considered. For instance a gene replacement therapy might by fruitful for patients carrying deletions or mutations in $\mathrm{G}^{124}$ of SURF1 but might be less efficient for patients with mutations affecting $\mathrm{Y}^{274}$ of SURF1. 


\subsection{SURF1 ${ }^{\mathrm{Y} 27 \mathrm{D}}$ reveals novel cytochrome $c$ oxidase assembly intermediates}

The assembly of the cytochrome $c$ oxidase from individual subunits of different genetic origin is a highly complex process, that involves more than 20 additional factors and is believed to function in an assembly-line like manner (Herrmann and Funes, 2005; Mick et al., 2011; Nijtmans et al., 1998). In yeast as a model system to study mitochondrial biogenesis, the cytochrome $c$ oxidase assembly has been studied extensively. The earliest assembly intermediates shown, are the Cox1-containing translational feed back regulation complexes, mentioned previously. In addition, also downstream assembly intermediates such as the Cox1-Cox3-Cox5-Cox6 complex in cells lacking Cox2 have been described (Horan et al., 2005). Although assembly intermediates of the cytochrome $c$ oxidase were also shown in human mitochondria (Oswald et al., 2009; Stiburek et al., 2005; Weraarpachai et al., 2009; Williams et al., 2004), their nature and constituents are less understood.

Interestingly, the early steps of cytochrome $c$ oxidase assembly are apparently not conserved in yeast and mammals and no robust mammalian homologs of yeast factors involved in expression of mitochondria-encoded subunits of the cytochrome $c$ oxidase can be found. This might be due to the fact that the mitochondrial mRNA structure differs significantly between yeast and mammals. Yeast mitochondrial mRNAs contain untranslated regions, which are bound by translational activators. In contrast, human mitochondrial mRNAs lack extensive untranslated regions. Interestingly, SURF1 and factors, required for cofactor insertion (Cox10, Cox11), might be the earliest assembly factors conserved in yeast and mammals.

Due to the differences in early steps of Cox 1 maturation and cytochrome $c$ oxidase assembly between yeast and mammals, to date little is known about these steps in mammalian mitochondria. In this study mild solubilization conditions (digitonin) were used to resolve SURF1 containing assembly intermediates of the cytochrome $c$ oxidase as shown for yeast Shyl (Mick et al., 2007). Although COX1 and SURF1 containing complexes could be detected at about $200 \mathrm{kDa}$, they apparently did not co-migrate. Using in vitro assembly analysis of SURF1 into isolated mitochondria however, it could be shown, that the SURF1 complex of about $200 \mathrm{kDa}$ depends on mitochondrial translation. Therefore, it was speculated that this complex contains mitochondrial translation products and represents a novel assembly intermediate of the cytochrome $c$ oxidase. Interestingly, this $200 \mathrm{kDa}$ complex of 
SURF1 is strongly increased when SURF1 $1^{\mathrm{Y} 274}$ is assembled in vitro, whereas the other SURF1 complexes are missing. The preferential accumulation of SURF1 ${ }^{\mathrm{Y} 274 \mathrm{D}}$ to this bona fide assembly intermediate of the cytochrome $c$ oxidase indicates an allele specific accumulation of a "dead-end" product of cytochrome $c$ oxidase assembly or a reduced offrate of SURF1 ${ }^{\mathrm{Y} 274 \mathrm{D}}$.

Eventually, SURF1 ${ }^{\mathrm{Y} 274 \mathrm{D}}$ blocks the cytochrome $c$ oxidase assembly at a distinct step. Subsequently, these assembly intermediates, not able to progress this bottleneck are degraded in patients, which would explain their lack of functional cytochrome $c$ oxidase. On the other hand, the accumulation of so far uncharacterized assembly intermediates could be an ideal tool for biochemical characterization of these intermediates and bring more insights into the assembly process of the mammalian cytochrome $c$ oxidase.

\subsection{Association of SURF1 with mature cytochrome $c$ oxidase}

The function of SURF1/Shy1 remains not fully understood. However, the association of Shy1 to assembly intermediates of the cytochrome $c$ oxidase, shown in this study and also reported previously (Mick et al., 2007; Pierrel et al., 2007) indicates a chaperone like function of SURF1/Shy1. This study provides evidence for a chaperone like function also for SURF1 since it associates to potential assembly intermediates of the mammalian cytochrome $c$ oxidase.

Interestingly it was shown in previous studies, that Shyl also associates to mature cytochrome $c$ oxidase (Mick et al., 2007). In this study, using in vitro assembly analysis and mild solubilization conditions, it could be shown that also SURF1 interacts with mature cytochrome $c$ oxidase in mammalian mitochondria. This finding is of especial interest, because it confirms the interaction of Shyl with mature yeast cytochrome $c$ oxidase and it also raises the question why a potential chaperone should interact with a mature complex. To date the function of the association of SURF1/Shy1 to mature complexes remains ill-defined. However, it could be speculated, that SURF1/Shy1 is involved in repair processes of the mature cytochrome $c$ oxidase. Such repair mechanisms of fully assembled complexes have been reported previously for the photosystem II, which similar to the cytochrome $c$ oxidase is a multi subunit complex involved in electron transport (Dobakova et al., 2007; Nowaczyk et al., 2006). 


\subsection{Shy $1^{\mathrm{Y} 344 \mathrm{D}}$ uncovers dual functionality of Shy1}

Shy 1 couples Cox 1 expression to cytochrome $c$ oxidase assembly in yeast mitochondria (Barrientos et al., 2002; Mick et al., 2007). Although Cox1 translational regulation in yeast mitochondria has been studied (Barrientos et al., 2004; Mick et al., 2010; Pierrel et al., 2007), the mechanism of coupling Cox1 translation to cytochrome $c$ oxidase assembly by Shy 1 is less understood.

Interestingly, shy $1^{Y 344 D}$ mitochondria showed wild-type-like expression of Cox1 but lack of cytochrome $c$ oxidase assembly. In this case Cox1 translational control is uncoupled from cytochrome $c$ oxidase assembly. Shy $1^{\mathrm{Y} 344 \mathrm{D}}$ releases Cox1 from translational feed back regulation complexes but does not allow further cytochrome $c$ oxidase assembly. Therefore, analysis of the shyl $1^{Y 344 D}$ mutation revealed dual functionality of Shyl.

Moreover, the shyl $1^{Y 344 D}$ allele represents an ideal tool to study the early and late steps of Shy1 dependent Cox1 maturation independent of each other. This was so far not possible, since lack of Shyl not only leads to defective cytochrome $c$ oxidase assembly but also shuts down Cox1 expression.

Interestingly, there are suppressor mutants of a SHY1-deletion (Barrientos et al., 2004; Bestwick et al., 2010a). These suppressors lead to overexpression of Cox1. The role of these suppressors in later steps of cytochrome $c$ oxidase assembly however, is unclear. In further studies, analysis of these suppressors in shyl $1^{Y 344 D}$ cells might help to understand and dissect the dual functionality of Shy1 in more detail.

\subsection{Degradation of assembly intermediates of the cytochrome c oxidase}

The biogenesis of the cytochrome $c$ oxidase has to be highly regulated to prevent accumulation of cytotoxic assembly intermediates, prone to production of reactive oxygen species (Khalimonchuk et al., 2007). Nevertheless, due to potential mutations affecting mitochondrial proteins or misfolding, an accumulation of such assembly intermediates cannot be excluded.

The $\mathrm{Y}^{274} \mathrm{D}$ exchange in SURF1 has been shown to alter the assembly process of the cytochrome $c$ oxidase, leading to an accumulation of potentially harmful cytochrome $c$ oxidase assembly intermediates. In fact, it has been shown that the corresponding yeast 
Shy $1^{\text {Y344D }}$ allows Cox1 conversion to its pro-oxidant state (Bestwick et al., 2010a). Moreover, the high somatic mutation rate of mitochondrial DNA (Brown et al., 1979) causes deleterious mutations affecting the mitochondria-encoded core subunits of the cytochrome $c$ oxidase. Therefore, not only a tight regulation of the biogenesis of the cytochrome $c$ oxidase, but also a repair mechanism or degradation system for impaired assembly intermediates is required. Interestingly, in this study it was shown that Cox1 is rapidly degraded when cytochrome $c$ oxidase assembly was impaired at later steps due to the shy $1^{Y 344 D}$ mutation. Moreover, it was shown that Shy $1^{\mathrm{Y} 344 \mathrm{D}}$, when overexpressed in the wild-type background, has a dominant negative effect on Cox1 stability, although no phenotype could be observed. Therefore, one could speculate, that in this case the accumulation of cytochrome $c$ oxidase assembly intermediates triggers the mitochondrial degradation system, which eliminates deleterious assembly intermediates and restores wild-type like growth. Interestingly, a heterozygous SURF $1^{Y 274 D} / S U R F 1^{W T}$ state does also not lead to Leigh Syndrome (Teraoka et al., 1999), again indicating an involvement of repair systems.

The described assembly defects and degradation of Cox1 due to the shy $1^{\text {Y344D }}$ (SURF1 $1^{\text {Y274D }) ~}$ mutation make this mutation an ideal tool to analyze these degradation processes in more detail in upcoming studies.

To date little is known about specific mitochondrial repair or degradation systems. It was reported that the m-AAA-protease (Arlt et al., 1998), as well as Omal (Bestwick et al., 2010b; Kaser et al., 2003), mitochondrial inner membrane proteases, are involved in Cox1 processing and degradation. Moreover, mitochondria can be degraded by autophagy. This autophagic degradation of mitochondria (mitophagy) could be involved in elimination of mitochondria that accumulate cytochrome $c$ oxidase assembly intermediates, which are potentially cytotoxic.

In this study, the influence of Uth1 on mitochondrial degradation was analyzed. However, in contrast to previous studies (Kissová et al., 2004), a resistance of $u t h 1 \Delta$ cells to rapamycin could not be observed. Hence, an involvement of Uth1 in autophagic processes could not be validated. Moreover, in vitro import analyses indicated a localization of Uth1 to the mitochondrial inner membrane rather than to the outer membrane as reported previously (Velours et al., 2002). Taken together this study provides evidence that Uth1 is not capable to function as a receptor for mitophagy in the mitochondrial outer membrane as previously speculated (Camougrand et al., 2003). The analysis of mechanisms that lead to degradation of 
damaged mitochondria is an interesting area of further research. It will also help to understand the response of cells to deleterious cytochrome $c$ oxidase assembly intermediates and accumulation of reactive oxygen species.

\subsection{Sym1 is a mitochondrial member of a conserved protein family}

In this study, Sym1 was used as a model to characterize the MPV17/Sym1 protein family, which is involved in the development of the Mitochondrial DNA Depletion Syndrome (Spinazzola et al., 2006). Interestingly, the MPV17 protein, first identified in mouse (Weiher et al., 1990), was initially found to be localized in peroxisomes (Zwacka et al., 1994). Later studies however, showed a mitochondrial localization of MPV17 (Spinazzola et al., 2006). In this study, it was confirmed that Sym1, the yeast MPV17 homolog, is exclusively located to mitochondria as previously reported (Trott and Morano, 2004).

Moreover, the submitochondrial localization and topology of Sym1 was characterized in this study indicating that Sym1 is located to the mitochondrial inner membrane. Furthermore, using transmembrane span predictions and protease protection analyses it could be concluded, that the $\mathrm{N}$ - and C-terminus as well as a central loop is exposed to the mitochondrial inter membrane space and 2 loops are exposed to the matrix (N-out/C-outtopology). Such a membrane integration has been proposed previously (Trott and Morano, 2004), the orientation however remained unclear and has been predicted inverse in previous studies (Wong et al., 2007).

The initial identification of MPV17 in mouse peroxisomes cannot be explained, however it should be noted, that also peroxisomal homologs of the MPV17/Sym1 family exist. These peroxisomal proteins, PMP22 in mammals and Yor292c in yeast, show sequence homology to Sym1/MPV17 and also have 4 predicted transmembrane spans. Interestingly a N-out/Cout-topology of PMP22 could be shown by accessibility to aminopeptidase (Kaldi et al., 1993). This indicates a conserved topology of peroxisomal and mitochondrial members of this protein family. Moreover, Sym1/MPV17 homologs were also found in woronin bodies, organelles of filamentous fungi that are derived from peroxisomes (Liu et al., 2008).

Taken together, Sym1/MPV17 represent mitochondrial members of a large ubiquitous family of conserved proteins, present in different organelles. Although a functional conservation of 
these proteins remains speculative, a better understanding of the function of Sym1/MPV17 might also shed light in the molecular functions of the other family members.

\subsection{A controversy about the sym1 $1 \Delta$ phenotype}

In contrast to previous studies (Dallabona et al., 2010; Spinazzola et al., 2006; Trott and Morano, 2004) no respiratory phenotype or sensitivity to ethanol was detected in $s y m 1 \Delta$ cells in this study. All previously reported phenotypes on ethanol-containing media at elevated temperatures were observed in strains derived from the BY4741/BY4743 background (Brachmann et al., 1998). However, in this study YPH499 was used as a background (Sikorski and Hieter, 1989).

Hence, it can be speculated that the different results are due to the use of other genetic backgrounds. This indicates that the suggested role of Sym1 in ethanol tolerance of yeast cells might be indirect.

A loss of mitochondrial DNA, as seen in patients with MPV17 mutations, could lead to respiratory deficiency of sym $1 \Delta$ cells. Interestingly, evidence for (Spinazzola et al., 2006) and against (Trott and Morano, 2004) increased loss of mitochondrial DNA in syml $\Delta$ cells was reported. A loss of mitochondrial DNA however, would not account for the ethanol and temperature specificity of this phenotype. Furthermore, high copy suppressors of a SYM1 deletion were reported, that increase protein kinase A activity (Trott and Morano, 2004). The activity of protein kinase A however, was not monitored in any of the studies.

It was reported that the ethanol sensitivity of $\operatorname{sym} 1 \Delta$ cells could be suppressed by addition of glucose (Trott and Morano, 2004). Therefore, a direct toxicity of ethanol can be excluded and the growth defect on ethanol containing plates at $37^{\circ} \mathrm{C}$ of $s y m 1 \Delta$ cells appears to be an indirect effect. This hypothesis is supported by a recent report (Dallabona et al., 2010) indicating that the sym1 $1 \Delta$ phenotype on lactate, acetate and low-glucose plates is similar to the phenotype on ethanol plates. The same report suggests defective glycogen storage, mitochondrial morphology and reduced activity of succinate dehydrogenase in sym $1 \Delta$ cells and therefore presents evidence for pleiotropic effects rather than for a specific phenotype of a SYM1 deletion.

Similar to the sym1 $1 \Delta$ phenotype that could not be reproduced in this study, also the kidney specific phenotype of Mpv17 $7^{-/}$mice (Weiher et al., 1990) was not reproducible as reported 
by Spinazzola et al. (2006). Hence, an adaptation to the loss of MPV17/Sym1 is feasible but awaits further experimental evidence.

\subsection{Sym1 complexes in the mitochondrial inner membrane}

Mitochondrial proteins of the inner membrane often assemble to multimeric structures in order to form functional complexes. Well-studied examples are the respiratory chain complexes or the protein translocase complexes of the mitochondrial inner membrane. Although for Sym1 an assembly into multimeric complexes was hypothesized (Trott and Morano, 2004), no experimental data for a Sym1 complex has been reported so far. In this study, mild solubilization of native protein complexes was used to separate these complexes in a polyacrylamide gel. Using Western blot analysis of tagged Sym1, it could be shown that Sym1 assembles into complexes of about 120 and $220 \mathrm{kDa}$. Subsequently, these complexes could even be purified in a native state. Moreover, an in vitro assembly assay of Sym1 complexes in yeast mitochondria could be established. All these experiments suggested the assembly of Sym1 into complexes of 120 and $220 \mathrm{kDa}$. The constituents of these complexes however remain unknown. In this regard, the isolation of these complexes, established in this study, might be an ideal tool to characterize their composition biochemically by mass spectrometry or Western blot analysis. The identification of interaction partners of Sym1 will not only reveal the structure and biogenesis of these complexes, it also will help to understand the physiological role and molecular function of the Sym1 complexes.

Interestingly, two complexes, $\mathrm{Sym} 1_{\mathrm{c} 120}$ and $\mathrm{Sym} 1_{\mathrm{c} 220}$, could be resolved by BN-PAGE analysis in this study. It can be speculated that both complexes are functionally relevant and have two different physiological roles in the mitochondrial inner membrane. However, it is also feasible that the $120 \mathrm{kDa}$ complex is either an assembly intermediate or a product of partial disassembly of the $220 \mathrm{kDa}$ complex due to solubilization.

For PMP22, the peroxisomal Sym1/MPV17 homolog, the formation of a homotrimeric structure was suggested (Rokka et al., 2009). Therefore, a homotrimeric structure might also be considered for Sym1/MPV17. In fact, since the potential monomeric Sym1 runs at about $65 \mathrm{kDa}$ in BN-PAGE analysis, the complexes at about $120 \mathrm{kDa}$ and $220 \mathrm{kDa}$ might represent dimers and trimers of Sym1, respectively. This speculation however, is not trivial to prove. Co-isolation of Sym1 proteins with different tags, or crosslinking approaches might be an option to study this in more detail in further analyses. 


\subsection{Sym1 as a model for the assembly of polytopic inner membrane proteins}

The import of nuclear-encoded proteins into mitochondria involves the translocases of the outer and inner mitochondrial membrane. Due to the diversity of membrane topologies and numbers of transmembrane segments of integral inner membrane proteins, multiple import pathways via TIM23 and TIM22 have evolved (see section 1.2.2). This raises the question how polytopic inner membrane proteins such as Sym1/MPV17 are translocated and inserted into the inner membrane.

Although an N-terminal presequence was predicted in MPV17/Sym1 previously (Spinazzola et al., 2006), in this study it could be shown that Sym1 lacks an N-terminal presequence and rather contains internal targeting sequences.

The establishment of an in vitro import and assembly assay allowed to resolve the assembly kinetics of Sym1. These revealed a rapid association of Sym1 to the respective complexes at 120 and $220 \mathrm{kDa}$. Furthermore, transient assembly intermediates were detected, which were speculated to be monomeric Sym1 as well as Sym1 associated to the TOM complex. In addition a complex accumulated in the absence of the membrane potential. Most likely this complex is a stage III import intermediate, also observed when other polytopic inner membrane proteins such as AAC are imported (Ryan et al., 1999).

Multispanning mitochondrial inner membrane proteins with internal targeting sequences, as the metabolite carriers, typically are transported via the TIM22 translocase (Becker et al., 2009; Rehling et al., 2004). Therefore, in this study it was initially assumed that Sym1 could be a TIM22 substrate. Import analyses into different TIM22 mutant mitochondria however, revealed that Sym1 is transported in a TIM22 independent manner. In contrast, it was shown that Sym1 import and assembly is dependent on TIM23. This unexpected finding indicates that Sym1 translocation into the mitochondrial inner membrane functions in an unconventional manner. Proteins, such as Bcs1, Mdj2 and Pam18, contain an internal presequence-like domain C-terminal to a hydrophobic sequence (Fölsch et al., 1996; Mokranjac et al., 2003; Westermann and Neupert, 1997). These domains are believed to form a hairpin loop that acts like an amphiphatic presequence for TIM23 mediated translocation. The mechanism of translocation of such proteins however, remains poorly understood. If such a presequence-like internal targeting sequence is also present in Sym1 remains 
speculative so far. Using truncation constructs of Sym1 for in vitro import experiments however, revealed that the targeting signal is located in the central part of Sym1.

Taken together, Sym1 takes an unusual route of import into the mitochondrial inner membrane. Moreover, Sym1 represents a model for import of proteins with four transmembrane spans, only few of which have been characterized so far. Therefore, Sym1 is an ideal model to study alternative import mechanisms of four transmembrane span proteins in more detail.

\subsection{SYM1 models for patient MPV17 point mutations}

The Mitochondrial DNA Depletion Syndrome can be caused by mutations in MPV17 (ElHattab et al., 2010; Spinazzola et al., 2006; Wong et al., 2007). In this disease, tissue specific loss of mitochondrial DNA leads to mitochondrial dysfunction. The mechanisms, how MPV17 mutations lead to loss of mitochondrial DNA however, remain unclear since the physiological role and molecular function of MPV17 is so far unknown.

In previous studies (Spinazzola et al., 2006) SYM1 has been used as a model to analyze point mutations in MPV17. There it was shown that SYM1 point mutations, corresponding to patient MPV17 mutations, lead to a phenotype similar to $s y m 1 \Delta$ cells or at least retardation of growth. Moreover it was shown, that MPV17 expression at least partially complements a deletion of SYM1.

Since the phenotype of sym $1 \Delta$ cells reported previously (Trott and Morano, 2004) was not reproducible in this study (see section 4.7), and a dominant negative effect of SYM1 alleles, mimicking patient MPV17 mutations, could not be detected, phenotypic characterization of these mutations cannot be performed in this analysis. However, destabilization of Sym $1^{\mathrm{R} 51 \mathrm{Q}}$ or Sym1 $1^{\mathrm{N} 172 \mathrm{~K}}$, mimicking patient MPV17 $7^{\mathrm{R} 50 \mathrm{Q}}$ and MPV17 ${ }^{\mathrm{N} 172 \mathrm{~K}}$, as speculated (Spinazzola et al., 2006) could be excluded by steady state analysis. Furthermore, using in vitro import analysis of $\left[{ }^{35} \mathrm{~S}\right]$ labeled Sym1 precursors, it could be shown that mutant Sym1 ${ }^{\mathrm{R} 51 \mathrm{Q}}$ and Sym $1^{\mathrm{N} 172 \mathrm{~K}}$ is not only imported into mitochondria, it also assembles to the same complexes as Sym $1^{\text {WT }}$. Therefore it can be concluded, that Sym $1^{\mathrm{R} 51 \mathrm{Q}}$ and $\mathrm{Sym} 1^{\mathrm{N} 172}$, similar to Shy1 ${ }^{\mathrm{Y} 344 \mathrm{D}}$ is not affected by mistargeting or degradation. Instead, mutation of the amino acids $\mathrm{R}^{50}$ and $\mathrm{N}^{166}$ in MPV17 ( $\mathrm{R}^{51}$ and $\mathrm{N}^{172}$ in Sym1) might lead to functional defects of the protein and subsequently to development of the Mitochondrial DNA Depletion Syndrome. 


\subsection{The channel activity of Sym1 and its implications for MDDS}

The function of Sym1/MPV17 in the mitochondrial inner membrane has been unknown so far. In contrast, the peroxisomal homolog PMP22 was shown to form a pore in the peroxisomal membrane (Rokka et al., 2009; Van Veldhoven et al., 1987).

In the present study, Sym1, isolated from yeast mitochondria, was characterized electrophysiologically. These analyses revealed a channel activity of Sym1, indicating that Sym1 forms a pore in the mitochondrial inner membrane. Although Sym1 showed a channel activity at all holding potentials tested, at physiological mitochondrial membrane potentials, which vary from $130-180 \mathrm{mV}$ (Chen, 1988; Hafner et al., 1990), increased dynamics of channel activity could be observed. This indicates dynamic properties of the Sym1 pore at physiological conditions.

The main conductance state of $450 \mathrm{pS}$ of the Sym1 channel corresponds to a pore size of about $1.6 \mathrm{~nm}$. This large pore size would enable free diffusion of metabolites as well as protons through the Sym1 pore. Interestingly, for PMP22 it was shown that metabolites of up to $300 \mathrm{Da}$ can pass the cannel (Rokka et al., 2009). However, free diffusion of protons across the mitochondrial inner membrane would disrupt the membrane potential and oxidative phosphorylation in mitochondria. Therefore, the Sym1 pore cannot exist as a constantly opened channel in the mitochondrial inner membrane. Hence, dynamic closure events of the channel on its own or by additional interaction partners can be postulated. The mechanisms and regulation of this channel closure could not be determined in this study and await further characterization.

The mutant $\mathrm{Sym} 1^{\mathrm{R} 51 \mathrm{Q}}$ and $\mathrm{Sym} 1^{\mathrm{N} 172 \mathrm{~K}}$ proteins might be interesting for further characterization of this channel, since defective channel function or regulation can be hypothesized. In fact, the patient MPV17 mutations might lead to proton leakage through the mitochondrial inner membrane. This would lead to an increased production of reactive oxygen species (Brookes, 2005; Turrens, 1997, 2003), which could account for damage and loss of mitochondrial DNA as seen in the patients. Production of reactive oxygen species due to mutations in MPV17 has been studied, however results of these analyses are inconsistent. There are reports of increased (Wagner et al., 2001), reduced (Zwacka et al., 1994) and 
unchanged (Trott and Morano, 2004) production of reactive oxygen species due to a loss of Sym1/MPV17. Thus, this has to be reevaluated.

The channel forming activity of Sym1 raises the question of the physiological role of this pore. A cargo of the Sym1 pore remains unknown, however determination of the reverse potential indicated selectivity for cations, suggesting a positively charged cargo. Furthermore, it can be speculated that the presence of Sym1/MPV17 homologs in peroxisomes, indicates a substrate that is present in both organelles. Biophysical and genetic interaction studies of Sym1 as well as more extensive phenotypical characterization of $s y m 1 \Delta$ cells might help to identify a cargo of the Sym1 pore in upcoming studies.

Members of the metabolite carrier family of the mitochondrial inner membrane have six transmembrane spans and facilitate pore formation upon dimerization (Palmieri and Pierri, 2010; Palmisano et al., 1998; Pebay-Peyroula et al., 2003). Tim23, a protein with four transmembrane spans, might also forms a pore as a homodimer (Bauer et al., 1996; Meinecke et al., 2006; Truscott et al., 2001). Hence, it is unlikely that the 4 transmembrane spans of Sym1 are sufficient to form a pore of $1.6 \mathrm{~nm}$. Therefore, it can be speculated that Sym1 forms a pore of this size upon complex formation or homo-oligomerization (see section 4.8).

Taken together, the identification of the channel activity of Sym1 revealed its molecular function as a pore in the mitochondrial inner membrane. The characterization of a cargo of the Sym1 pore in further studies will uncover the physiological role of Sym1. This will help to understand the pathogenesis of MPV17 associated Mitochondrial DNA Depletion Syndrome. 


\subsection{Conclusion}

In this thesis, nuclear-encoded mitochondrial proteins, involved in human disease were analyzed using yeast as a model system. Thereby the molecular pathology of SURF1 point mutations identified in Leigh Syndrome patients could be resolved. Mutations in $\mathrm{G}^{124}$ of SURF1 lead to degradation of the protein whereas an $\mathrm{Y}^{274} \mathrm{D}$ exchange in SURF1 ( $\mathrm{Y}^{344} \mathrm{D}$ in Shy1) led to impaired function of the protein. Moreover, the SURF1 $1^{Y 274 D} /$ shy $1^{Y 344 D}$ mutations indicate dual functionality of SURF1/Shy1 and represent ideal tools to resolve the assembly of the cytochrome $c$ oxidase in more detail.

Additionally, the biogenesis and molecular function of Sym1, the yeast homolog of MPV17, a protein involved in the Mitochondrial DNA Depletion Syndrome, could be characterized. It could be shown that Sym1 is imported in a TIM23 dependent manner into mitochondria and associates into high molecular complexes. Moreover, it was shown that Sym1 forms a pore in the mitochondrial inner membrane.

Taken together, it must be concluded that the analysis of human mitochondrial diseases, using yeast as a model can help to understand the molecular pathology of these diseases. A combination of clinical and biochemical findings will not only help to understand the biogenesis and function of mitochondria in more detail, it will also help to counteract mitochondrial diseases in a systematic manner. 


\section{Summary}

Mitochondrial diseases are fatal disorders mainly affecting highly energy dependent tissues such as brain, heart, liver and muscle. These severe disorders can be caused by mutations affecting mitochondrial- or nuclear-encoded proteins. The molecular function of many nuclear-encoded mitochondrial proteins involved in disease is unknown so far and therefore, the mechanisms that lead to disease are enigmatic. In this study, point mutations in SURF1 and MPVI7 were analyzed that were found in patients of the Leigh Syndrome and the Mitochondrial DNA Depletion Syndrome respectively. These missense mutations affect highly conserved and therefore potentially functional important amino acids.

The function of SURF1, a highly conserved early assembly factor of the cytochrome $c$ oxidase, has been studied using its yeast homolog Shyl. Shyl is involved in the maturation and translational feed back regulation of Cox1, the central subunit of the cytochrome $c$ oxidase. In this study, it was shown that patient point mutations affecting $\mathrm{G}^{124}$ in SURF1 lead to rapid degradation of the protein after mitochondrial import. In contrast, patient mutations that lead to an $\mathrm{Y}^{274} \mathrm{D}$ exchange in SURF1 do not alter mitochondrial import or stability but showed increased association to a newly identified cytochrome $c$ oxidase assembly intermediate. Hence, SURF1 ${ }^{\mathrm{Y} 274 \mathrm{D}}$ shows impaired function. Using the corresponding yeast Shy $1^{\text {Y344D }}$ as a model, dual functionality of SURF1/Shy1 could be shown. Shy1 ${ }^{\mathrm{Y} 344 \mathrm{D}}$ releases cytochrome $c$ oxidase assembly intermediates from translational feed back regulation of Cox 1 but fails to promote cytochrome $c$ oxidase assembly at later steps.

Mutations affecting MPV17, a mitochondrial inner membrane protein of previously unknown function, lead to loss of mitochondrial DNA. In this study Sym1, the yeast homolog of MPV17, was used as a model to study the biogenesis and function of MPV17/Sym1. It could be shown that Sym1 forms high molecular weight complexes of 120 and $220 \mathrm{kDa}$ in the mitochondrial inner membrane. In contrast to most polytopic inner membrane proteins, Sym1 is imported in a TIM23 dependent manner into mitochondria. The function of Sym1 was analyzed by electrophysiological characterization of Sym1, isolated from yeast mitochondria. Sym1 showed channel activity, which indicates a pore forming function of Sym1/MPV17 in the mitochondrial inner membrane. 


\section{Bibliography}

Acin-Perez, R., Fernandez-Silva, P., Peleato, M.L., Perez-Martos, A., and Enriquez, J.A. (2008). Respiratory active mitochondrial supercomplexes. Mol Cell 32, 529-539.

Ahting, U., Thieffry, M., Engelhardt, H., Hegerl, R., Neupert, W., and Nussberger, S. (2001). Tom40, the poreforming component of the protein-conducting TOM channel in the outer membrane of mitochondria. J Cell Biol 153, 1151-1160.

Ajioka, R.S., Phillips, J.D., and Kushner, J.P. (2006). Biosynthesis of heme in mammals. Biochim Biophys Acta $1763,723-736$.

Alexander, C., Votruba, M., Pesch, U.E., Thiselton, D.L., Mayer, S., Moore, A., Rodriguez, M., Kellner, U., Leo-Kottler, B., Auburger, G., et al. (2000). OPA1, encoding a dynamin-related GTPase, is mutated in autosomal dominant optic atrophy linked to chromosome 3q28. Nat Genet 26, 211-215.

Andersson, S.G., Zomorodipour, A., Andersson, J.O., Sicheritz-Ponten, T., Alsmark, U.C., Podowski, R.M., Naslund, A.K., Eriksson, A.S., Winkler, H.H., and Kurland, C.G. (1998). The genome sequence of Rickettsia prowazekii and the origin of mitochondria. Nature 396, 133-140.

Arlt, H., Steglich, G., Perryman, R., Guiard, B., Neupert, W., and Langer, T. (1998). The formation of respiratory chain complexes in mitochondria is under the proteolytic control of the m-AAA protease. EMBO J $17,4837-4847$.

Arnold, I., Pfeiffer, K., Neupert, W., Stuart, R.A., and Schägger, H. (1998). Yeast mitochondrial F1F0-ATP synthase exists as a dimer: identification of three dimer-specific subunits. EMBO J 17, 7170-7178.

Balaban, R.S., Nemoto, S., and Finkel, T. (2005). Mitochondria, oxidants, and aging. Cell 120, 483-495.

Barnett, J.A. (1998). A history of research on yeasts. 1: Work by chemists and biologists 1789-1850. Yeast 14, 1439-1451.

Barnett, J.A. (2000). A history of research on yeasts 2: Louis Pasteur and his contemporaries, 1850-1880. Yeast $16,755-771$.

Barrientos, A. (2003). Yeast models of human mitochondrial diseases. IUBMB Life 55, 83-95.

Barrientos, A., Korr, D., and Tzagoloff, A. (2002). Shy1p is necessary for full expression of mitochondrial COX1 in the yeast model of Leigh's syndrome. EMBO J 21, 43-52.

Barrientos, A., Zambrano, A., and Tzagoloff, A. (2004). Mss51p and Cox14p jointly regulate mitochondrial Cox $1 p$ expression in Saccharomyces cerevisiae. EMBO J 23, 3472-3482.

Barth, P.G., Scholte, H.R., Berden, J.A., Van der Klei-Van Moorsel, J.M., Luyt-Houwen, I.E., Van 't VeerKorthof, E.T., Van der Harten, J.J., and Sobotka-Plojhar, M.A. (1983). An X-linked mitochondrial disease affecting cardiac muscle, skeletal muscle and neutrophil leucocytes. J Neurol Sci 62, 327-355.

Barth, P.G., Valianpour, F., Bowen, V.M., Lam, J., Duran, M., Vaz, F.M., and Wanders, R.J. (2004). X-linked cardioskeletal myopathy and neutropenia (Barth syndrome): an update. Am J Med Genet A 126A, 349-354.

Bauer, M.F., Sirrenberg, C., Neupert, W., and Brunner, M. (1996). Role of Tim23 as voltage sensor and presequence receptor in protein import into mitochondria. Cell 87, 33-41. 
Becker, L., Bannwarth, M., Meisinger, C., Hill, K., Model, K., Krimmer, T., Casadio, R., Truscott, K.N., Schulz, G.E., Pfanner, N., et al. (2005). Preprotein translocase of the outer mitochondrial membrane: reconstituted Tom40 forms a characteristic TOM pore. J Mol Biol 353, 1011-1020.

Becker, T., Gebert, M., Pfanner, N., and van der Laan, M. (2009). Biogenesis of mitochondrial membrane proteins. Curr Opin Cell Biol 21, 484-493.

Becker, T., Vogtle, F.N., Stojanovski, D., and Meisinger, C. (2008). Sorting and assembly of mitochondrial outer membrane proteins. Biochim Biophys Acta 1777, 557-563.

Bestwick, M., Jeong, M.-Y., Khalimonchuk, O., Kim, H., and Winge, D.R. (2010a). Analysis of Leigh syndrome mutations in the yeast SURF1 homolog reveals a new member of the cytochrome oxidase assembly factor family. Mol Cell Biol 30, 4480-4491.

Bestwick, M., Khalimonchuk, O., Pierrel, F., and Winge, D.R. (2010b). The role of Coa2 in hemylation of yeast Cox 1 revealed by its genetic interaction with Cox10. Mol Cell Biol 30, 172-185.

Bohnert, M., Rehling, P., Guiard, B., Herrmann, J.M., Pfanner, N., and van der Laan, M. (2010). Cooperation of stop-transfer and conservative sorting mechanisms in mitochondrial protein transport. Curr Biol 20, 1227-1232.

Brachmann, C.B., Davies, A., Cost, G.J., Caputo, E., Li, J., Hieter, P., and Boeke, J.D. (1998). Designer deletion strains derived from Saccharomyces cerevisiae S288C: a useful set of strains and plasmids for PCR-mediated gene disruption and other applications. Yeast 14, 115-132.

Brandt, U. (2006). Energy converting NADH:quinone oxidoreductase (complex I). Annu Rev Biochem 75, 6992.

Brookes, P.S. (2005). Mitochondrial H(+) leak and ROS generation: an odd couple. Free Radic Biol Med 38, $12-23$.

Brown, R.M., and Brown, G.K. (1996). Complementation analysis of systemic cytochrome oxidase deficiency presenting as Leigh syndrome. J Inherit Metab Dis 19, 752-760.

Brown, W.M., George, M., Jr., and Wilson, A.C. (1979). Rapid evolution of animal mitochondrial DNA. Proc Natl Acad Sci U S A 76, 1967-1971.

Bundschuh, F.A., Hannappel, A., Anderka, O., and Ludwig, B. (2009). Surf1, associated with Leigh syndrome in humans, is a heme-binding protein in bacterial oxidase biogenesis. J Biol Chem 284, 25735-25741.

Bundschuh, F.A., Hoffmeier, K., and Ludwig, B. (2008). Two variants of the assembly factor Surf1 target specific terminal oxidases in Paracoccus denitrificans. Biochim Biophys Acta 1777, 1336-1343.

Camougrand, N., Grelaud-Coq, A., Marza, E., Priault, M., Bessoule, J.J., and Manon, S. (2003). The product of the UTH1 gene, required for Bax-induced cell death in yeast, is involved in the response to rapamycin. Mol Microbiol 47, 495-506.

Carr, H.S., and Winge, D.R. (2003). Assembly of cytochrome c oxidase within the mitochondrion. Acc Chem Res 36, 309-316.

Carroll, J., Fearnley, I.M., Skehel, J.M., Shannon, R.J., Hirst, J., and Walker, J.E. (2006). Bovine complex I is a complex of 45 different subunits. J Biol Chem 281, 32724-32727.

Castresana, J., Lubben, M., Saraste, M., and Higgins, D.G. (1994). Evolution of cytochrome oxidase, an enzyme older than atmospheric oxygen. EMBO J 13, 2516-2525. 
Chacinska, A., Koehler, C.M., Milenkovic, D., Lithgow, T., and Pfanner, N. (2009). Importing mitochondrial proteins: machineries and mechanisms. Cell 138, 628-644.

Chacinska, A., Lind, M., Frazier, A.E., Dudek, J., Meisinger, C., Geissler, A., Sickmann, A., Meyer, H.E., Truscott, K.N., Guiard, B., et al. (2005). Mitochondrial presequence translocase: switching between TOM tethering and motor recruitment involves Tim21 and Tim17. Cell 120,817-829.

Chen, L.B. (1988). Mitochondrial membrane potential in living cells. Annu Rev Cell Biol 4, 155-181.

Clapham, D.E. (2007). Calcium signaling. Cell 131, 1047-1058.

Claypool, S.M., McCaffery, J.M., and Koehler, C.M. (2006). Mitochondrial mislocalization and altered assembly of a cluster of Barth syndrome mutant tafazzins. J Cell Biol 174, 379-390.

Coenen, M.J., Smeitink, J.A., Pots, J.M., van Kaauwen, E., Trijbels, F.J., Hol, F.A., and van den Heuvel, L.P. (2006). Sequence analysis of the structural nuclear encoded subunits and assembly genes of cytochrome $\mathrm{c}$ oxidase in a cohort of 10 isolated complex IV-deficient patients revealed five mutations. J Child Neurol 21, 508511.

Coenen, M.J., van den Heuvel, L.P., Nijtmans, L.G., Morava, E., Marquardt, I., Girschick, H.J., Trijbels, F.J., Grivell, L.A., and Smeitink, J.A. (1999). SURFEIT-1 gene analysis and two-dimensional blue native gel electrophoresis in cytochrome c oxidase deficiency. Biochem Biophys Res Commun 265, 339-344.

Copeland, W.C. (2008). Inherited mitochondrial diseases of DNA replication. Annu Rev Med 59, 131-146.

Curran, S.P., Leuenberger, D., Oppliger, W., and Koehler, C.M. (2002a). The Tim9p-Tim10p complex binds to the transmembrane domains of the ADP/ATP carrier. EMBO J 21, 942-953.

Curran, S.P., Leuenberger, D., Schmidt, E., and Koehler, C.M. (2002b). The role of the Tim8p-Tim13p complex in a conserved import pathway for mitochondrial polytopic inner membrane proteins. J Cell Biol 158, 1017 1027.

Dallabona, C., Marsano, R.M., Arzuffi, P., Ghezzi, D., Mancini, P., Zeviani, M., Ferrero, I., and Donnini, C. (2010). Sym1, the yeast ortholog of the MPV17 human disease protein, is a stress-induced bioenergetic and morphogenetic mitochondrial modulator. Hum Mol Genet 19, 1098-1107.

Davis, A.J., Alder, N.N., Jensen, R.E., and Johnson, A.E. (2007). The Tim9p/10p and Tim8p/13p complexes bind to specific sites on Tim23p during mitochondrial protein import. Mol Biol Cell 18, 475-486.

de Duve, C. (2005). The lysosome turns fifty. Nat Cell Biol 7, 847-849.

Decoster, E., Simon, M., Hatat, D., and Faye, G. (1990). The MSS51 gene product is required for the translation of the COX1 mRNA in yeast mitochondria. Mol Gen Genet 224, 111-118.

Dekker, P.J., Martin, F., Maarse, A.C., Bömer, U., Müller, H., Guiard, B., Meijer, M., Rassow, J., and Pfanner, N. (1997). The Tim core complex defines the number of mitochondrial translocation contact sites and can hold arrested preproteins in the absence of matrix Hsp70-Tim44. EMBO J 16, 5408-5419.

Delettre, C., Lenaers, G., Griffoin, J.M., Gigarel, N., Lorenzo, C., Belenguer, P., Pelloquin, L., Grosgeorge, J., Turc-Carel, C., Perret, E., et al. (2000). Nuclear gene OPA1, encoding a mitochondrial dynamin-related protein, is mutated in dominant optic atrophy. Nat Genet 26, 207-210.

DiMauro, S., and Schon, E.A. (2008). Mitochondrial disorders in the nervous system. Annu Rev Neurosci 31, 91-123. 
Dobakova, M., Tichy, M., and Komenda, J. (2007). Role of the PsbI protein in photosystem II assembly and repair in the cyanobacterium Synechocystis sp. PCC 6803. Plant Physiol 145, 1681-1691.

du Plessis, D.J., Nouwen, N., and Driessen, A.J. (2011). The Sec translocase. Biochim Biophys Acta 1808, 851865.

Duhig, T., Ruhrberg, C., Mor, O., and Fried, M. (1998). The human Surfeit locus. Genomics 52, 72-78.

El-Hattab, A.W., Li, F.-Y., Schmitt, E., Zhang, S., Craigen, W.J., and Wong, L.-J.C. (2010). MPV17-associated hepatocerebral mitochondrial DNA depletion syndrome: new patients and novel mutations. Mol Genet Metab 99, 300-308.

Fabrizi, G.M., Sadlock, J., Hirano, M., Mita, S., Koga, Y., Rizzuto, R., Zeviani, M., and Schon, E.A. (1992). Differential expression of genes specifying two isoforms of subunit VIa of human cytochrome c oxidase. Gene $119,307-312$.

Ferguson-Miller, S., and Babcock, G.T. (1996). Heme/Copper Terminal Oxidases. Chem Rev 96, 2889-2908.

Finsterer, J. (2004). Mitochondriopathies. Eur J Neurol 11, 163-186.

Finsterer, J. (2008). Leigh and Leigh-like syndrome in children and adults. Pediatr Neurol 39, 223-235.

Fölsch, H., Guiard, B., Neupert, W., and Stuart, R.A. (1996). Internal targeting signal of the BCS1 protein: a novel mechanism of import into mitochondria. EMBO J 15, 479-487.

Fontanesi, F., Clemente, P., and Barrientos, A. (2011). Cox25 teams up with Mss51, Ssc1, and Cox14 to regulate mitochondrial cytochrome c oxidase subunit 1 expression and assembly in Saccharomyces cerevisiae. $\mathrm{J}$ Biol Chem 286, 555-566.

Gebert, N., Chacinska, A., Wagner, K., Guiard, B., Koehler, C.M., Rehling, P., Pfanner, N., and Wiedemann, N. (2008). Assembly of the three small Tim proteins precedes docking to the mitochondrial carrier translocase. EMBO Rep 9, 548-554.

Geissler, A., Chacinska, A., Truscott, K.N., Wiedemann, N., Brandner, K., Sickmann, A., Meyer, H.E., Meisinger, C., Pfanner, N., and Rehling, P. (2002). The mitochondrial presequence translocase: an essential role of Tim50 in directing preproteins to the import channel. Cell 111, 507-518.

Gennis, R.B. (1998). How does cytochrome oxidase pump protons? Proc Natl Acad Sci U S A 95, 1274712749.

Gietz, R.D., and Schiestl, R.H. (2007). High-efficiency yeast transformation using the LiAc/SS carrier DNA/PEG method. Nat Protoc 2, 31-34.

Gilderson, G., Salomonsson, L., Aagaard, A., Gray, J., Brzezinski, P., and Hosler, J. (2003). Subunit III of cytochrome c oxidase of Rhodobacter sphaeroides is required to maintain rapid proton uptake through the D pathway at physiologic $\mathrm{pH}$. Biochemistry $42,7400-7409$.

Glick, B.S., Brandt, A., Cunningham, K., Muller, S., Hallberg, R.L., and Schatz, G. (1992). Cytochromes c1 and b2 are sorted to the intermembrane space of yeast mitochondria by a stop-transfer mechanism. Cell 69, 809-822.

Gogvadze, V., Orrenius, S., and Zhivotovsky, B. (2008). Mitochondria in cancer cells: what is so special about them? Trends Cell Biol 18, 165-173.

Gray, M.W., Burger, G., and Lang, B.F. (1999). Mitochondrial evolution. Science 283, 1476-1481. 
Graziewicz, M.A., Longley, M.J., and Copeland, W.C. (2006). DNA polymerase gamma in mitochondrial DNA replication and repair. Chem Rev 106, 383-405.

Guarente, L. (2008). Mitochondria--a nexus for aging, calorie restriction, and sirtuins? Cell 132, 171-176.

Hafner, R.P., Brown, G.C., and Brand, M.D. (1990). Analysis of the control of respiration rate, phosphorylation rate, proton leak rate and protonmotive force in isolated mitochondria using the 'top-down' approach of metabolic control theory. Eur J Biochem 188, 313-319.

Hagerhall, C. (1997). Succinate: quinone oxidoreductases. Variations on a conserved theme. Biochim Biophys Acta $1320,107-141$.

Hansen, J.J., Durr, A., Cournu-Rebeix, I., Georgopoulos, C., Ang, D., Nielsen, M.N., Davoine, C.S., Brice, A., Fontaine, B., Gregersen, N., et al. (2002). Hereditary spastic paraplegia SPG13 is associated with a mutation in the gene encoding the mitochondrial chaperonin Hsp60. Am J Hum Genet 70, 1328-1332.

Heitman, J., Movva, N.R., and Hall, M.N. (1991). Targets for cell cycle arrest by the immunosuppressant rapamycin in yeast. Science $253,905-909$.

Hell, K., Herrmann, J.M., Pratje, E., Neupert, W., and Stuart, R.A. (1998). Oxa1p, an essential component of the N-tail protein export machinery in mitochondria. Proc Natl Acad Sci U S A 95, 2250-2255.

Hell, K., Neupert, W., and Stuart, R.A. (2001). Oxa1p acts as a general membrane insertion machinery for proteins encoded by mitochondrial DNA. EMBO J 20, 1281-1288.

Herrmann, J.M., and Funes, S. (2005). Biogenesis of cytochrome oxidase-sophisticated assembly lines in the mitochondrial inner membrane. Gene 354, 43-52.

Herrmann, J.M., and Köhl, R. (2007). Catch me if you can! Oxidative protein trapping in the intermembrane space of mitochondria. J Cell Biol 176, 559-563.

Hodge, M.R., Kim, G., Singh, K., and Cumsky, M.G. (1989). Inverse regulation of the yeast COX5 genes by oxygen and heme. Mol Cell Biol 9, 1958-1964.

Holt, I.J., Harding, A.E., and Morgan-Hughes, J.A. (1988). Deletions of muscle mitochondrial DNA in patients with mitochondrial myopathies. Nature 331, 717-719.

Holt, I.J., Harding, A.E., Petty, R.K., and Morgan-Hughes, J.A. (1990). A new mitochondrial disease associated with mitochondrial DNA heteroplasmy. Am J Hum Genet 46, 428-433.

Horan, S., Bourges, I., Taanman, J.W., and Meunier, B. (2005). Analysis of COX2 mutants reveals cytochrome oxidase subassemblies in yeast. Biochem J 390, 703-708.

Hudson, G., and Chinnery, P.F. (2006). Mitochondrial DNA polymerase-gamma and human disease. Hum Mol Genet 15 Spec No 2, R244-252.

Ito, H., Fukuda, Y., Murata, K., and Kimura, A. (1983). Transformation of intact yeast cells treated with alkali cations. J Bacteriol 153, 163-168.

Iwata, S., Lee, J.W., Okada, K., Lee, J.K., Iwata, M., Rasmussen, B., Link, T.A., Ramaswamy, S., and Jap, B.K. (1998). Complete structure of the 11-subunit bovine mitochondrial cytochrome bc1 complex. Science 281, 6471.

Iwata, S., Ostermeier, C., Ludwig, B., and Michel, H. (1995). Structure at 2.8 A resolution of cytochrome c oxidase from Paracoccus denitrificans. Nature 376, 660-669. 
Jamieson, D.J. (1998). Oxidative stress responses of the yeast Saccharomyces cerevisiae. Yeast 14, 1511-1527.

Janke, C., Magiera, M.M., Rathfelder, N., Taxis, C., Reber, S., Maekawa, H., Moreno-Borchart, A., Doenges, G., Schwob, E., Schiebel, E., et al. (2004). A versatile toolbox for PCR-based tagging of yeast genes: new fluorescent proteins, more markers and promoter substitution cassettes. Yeast 21, 947-962.

Kaji, S., Murayama, K., Nagata, I., Nagasaka, H., Takayanagi, M., Ohtake, A., Iwasa, H., Nishiyama, M., Okazaki, Y., Harashima, H., et al. (2009). Fluctuating liver functions in siblings with MPV17 mutations and possible improvement associated with dietary and pharmaceutical treatments targeting respiratory chain complex II. Mol Genet Metab 97, 292-296.

Kaldi, K., Diestelkötter, P., Stenbeck, G., Auerbach, S., Jäkle, U., Mägert, H.J., Wieland, F.T., and Just, W.W. (1993). Membrane topology of the $22 \mathrm{kDa}$ integral peroxisomal membrane protein. FEBS Lett 315, $217-222$.

Kametaka, S., Matsuura, A., Wada, Y., and Ohsumi, Y. (1996). Structural and functional analyses of APG5, a gene involved in autophagy in yeast. Gene 178, 139-143.

Kanki, T., and Klionsky, D.J. (2010). The molecular mechanism of mitochondria autophagy in yeast. Mol Microbiol 75, 795-800.

Kaser, M., Kambacheld, M., Kisters-Woike, B., and Langer, T. (2003). Oma1, a novel membrane-bound metallopeptidase in mitochondria with activities overlapping with the m-AAA protease. J Biol Chem 278, 46414-46423.

Kaukonen, J., Juselius, J.K., Tiranti, V., Kyttala, A., Zeviani, M., Comi, G.P., Keranen, S., Peltonen, L., and Suomalainen, A. (2000). Role of adenine nucleotide translocator 1 in mtDNA maintenance. Science 289, 782785.

Kellis, M., Patterson, N., Endrizzi, M., Birren, B., and Lander, E.S. (2003). Sequencing and comparison of yeast species to identify genes and regulatory elements. Nature 423, 241-254.

Kerscher, S.J. (2000). Diversity and origin of alternative NADH:ubiquinone oxidoreductases. Biochim Biophys Acta $1459,274-283$.

Khalimonchuk, O., Bestwick, M., Meunier, B., Watts, T.C., and Winge, D.R. (2010). Formation of the redox cofactor centers during Cox1 maturation in yeast cytochrome oxidase. Mol Cell Biol 30, 1004-1017.

Khalimonchuk, O., Bird, A., and Winge, D.R. (2007). Evidence for a pro-oxidant intermediate in the assembly of cytochrome oxidase. J Biol Chem 282, 17442-17449.

Khalimonchuk, O., and Rödel, G. (2005). Biogenesis of cytochrome c oxidase. Mitochondrion 5, 363-388.

Kissová, I., Deffieu, M., Manon, S., and Camougrand, N. (2004). Uth1p is involved in the autophagic degradation of mitochondria. J Biol Chem 279, 39068-39074.

Kohler, R., Boehringer, D., Greber, B., Bingel-Erlenmeyer, R., Collinson, I., Schaffitzel, C., and Ban, N. (2009). YidC and Oxal form dimeric insertion pores on the translating ribosome. Mol Cell 34, 344-353.

Laemmli, U.K. (1970). Cleavage of structural proteins during the assembly of the head of bacteriophage T4. Nature 227, 680-685.

Lazarou, M., Smith, S.M., Thorburn, D.R., Ryan, M.T., and McKenzie, M. (2009). Assembly of nuclear DNAencoded subunits into mitochondrial complex IV, and their preferential integration into supercomplex forms in patient mitochondria. FEBS J 276, 6701-6713. 
Leigh, D. (1951). Subacute necrotizing encephalomyelopathy in an infant. J Neurol Neurosurg Psychiatry 14 , 216-221.

Leuenberger, D., Bally, N.A., Schatz, G., and Koehler, C.M. (1999). Different import pathways through the mitochondrial intermembrane space for inner membrane proteins. EMBO J 18, 4816-4822.

Liesa, M., Palacin, M., and Zorzano, A. (2009). Mitochondrial dynamics in mammalian health and disease. Physiol Rev 89, 799-845.

Lill, R. (2009). Function and biogenesis of iron-sulphur proteins. Nature 460, 831-838.

Lithgow, T., and Schneider, A. (2010). Evolution of macromolecular import pathways in mitochondria, hydrogenosomes and mitosomes. Philos Trans R Soc Lond B Biol Sci 365, 799-817.

Liu, F., Ng, S.K., Lu, Y., Low, W., Lai, J., and Jedd, G. (2008). Making two organelles from one: Woronin body biogenesis by peroxisomal protein sorting. J Cell Biol 180, 325-339.

Luttik, M.A., Overkamp, K.M., Kotter, P., de Vries, S., van Dijken, J.P., and Pronk, J.T. (1998). The Saccharomyces cerevisiae NDE1 and NDE2 genes encode separate mitochondrial NADH dehydrogenases catalyzing the oxidation of cytosolic NADH. J Biol Chem 273, 24529-24534.

Luzio, J.P., Pryor, P.R., and Bright, N.A. (2007). Lysosomes: fusion and function. Nat Rev Mol Cell Biol 8, 622-632.

Manthey, G.M., and McEwen, J.E. (1995). The product of the nuclear gene PET309 is required for translation of mature mRNA and stability or production of intron-containing RNAs derived from the mitochondrial COX1 locus of Saccharomyces cerevisiae. EMBO J 14, 4031-4043.

Marres, C.A., de Vries, S., and Grivell, L.A. (1991). Isolation and inactivation of the nuclear gene encoding the rotenone-insensitive internal NADH: ubiquinone oxidoreductase of mitochondria from Saccharomyces cerevisiae. Eur J Biochem 195, 857-862.

Mashkevich, G., Repetto, B., Glerum, D.M., Jin, C., and Tzagoloff, A. (1997). SHY1, the yeast homolog of the mammalian SURF-1 gene, encodes a mitochondrial protein required for respiration. J Biol Chem 272, 1435614364.

McFarland, R., Taylor, R.W., and Turnbull, D.M. (2002). The neurology of mitochondrial DNA disease. Lancet Neurol 1, 343-351.

Meinecke, M., Wagner, R., Kovermann, P., Guiard, B., Mick, D.U., Hutu, D.P., Voos, W., Truscott, K.N., Chacinska, A., Pfanner, N., et al. (2006). Tim50 maintains the permeability barrier of the mitochondrial inner membrane. Science 312, 1523-1526.

Meisinger, C., Pfanner, N., and Truscott, K.N. (2006). Isolation of yeast mitochondria. Methods Mol Biol 313 , 33-39.

Mekhail, K., and Moazed, D. (2010). The nuclear envelope in genome organization, expression and stability. Nat Rev Mol Cell Biol 11, 317-328.

Merante, F., Petrova-Benedict, R., MacKay, N., Mitchell, G., Lambert, M., Morin, C., De Braekeleer, M., Laframboise, R., Gagne, R., and Robinson, B.H. (1993). A biochemically distinct form of cytochrome oxidase (COX) deficiency in the Saguenay-Lac-Saint-Jean region of Quebec. Am J Hum Genet 53, 481-487.

Mick, D.U., Fox, T.D., and Rehling, P. (2011). Inventory control: cytochrome c oxidase assembly regulates mitochondrial translation. Nat Rev Mol Cell Biol 12, 14-20. 
Mick, D.U., Vukotic, M., Piechura, H., Meyer, H.E., Warscheid, B., Deckers, M., and Rehling, P. (2010). Coa3 and Cox14 are essential for negative feedback regulation of COX1 translation in mitochondria. J Cell Biol 191, 141-154.

Mick, D.U., Wagner, K., van der Laan, M., Frazier, A.E., Perschil, I., Pawlas, M., Meyer, H.E., Warscheid, B., and Rehling, P. (2007). Shy1 couples Cox 1 translational regulation to cytochrome c oxidase assembly. EMBO J $26,4347-4358$.

Miller, C., Saada, A., Shaul, N., Shabtai, N., Ben-Shalom, E., Shaag, A., Hershkovitz, E., and Elpeleg, O. (2004). Defective mitochondrial translation caused by a ribosomal protein (MRPS16) mutation. Ann Neurol 56, 734-738.

Model, K., Meisinger, C., and Kuhlbrandt, W. (2008). Cryo-electron microscopy structure of a yeast mitochondrial preprotein translocase. J Mol Biol 383, 1049-1057.

Mokranjac, D., Popov-Celeketic, D., Hell, K., and Neupert, W. (2005). Role of Tim21 in mitochondrial translocation contact sites. J Biol Chem 280, 23437-23440.

Mokranjac, D., Sichting, M., Neupert, W., and Hell, K. (2003). Tim14, a novel key component of the import motor of the TIM23 protein translocase of mitochondria. EMBO J 22, 4945-4956.

Moraes, C.T., Shanske, S., Tritschler, H.J., Aprille, J.R., Andreetta, F., Bonilla, E., Schon, E.A., and DiMauro, S. (1991). mtDNA depletion with variable tissue expression: a novel genetic abnormality in mitochondrial diseases. Am J Hum Genet 48, 492-501.

Nakamoto, R.K., Baylis Scanlon, J.A., and Al-Shawi, M.K. (2008). The rotary mechanism of the ATP synthase. Arch Biochem Biophys 476, 43-50.

Nargang, F.E., Preuss, M., Neupert, W., and Herrmann, J.M. (2002). The Oxa1 protein forms a homooligomeric complex and is an essential part of the mitochondrial export translocase in Neurospora crassa. J Biol Chem 277, 12846-12853.

Neuhoff, V., Arold, N., Taube, D., and Ehrhardt, W. (1988). Improved staining of proteins in polyacrylamide gels including isoelectric focusing gels with clear background at nanogram sensitivity using Coomassie Brilliant Blue G-250 and R-250. Electrophoresis 9, 255-262.

Nijtmans, L.G., Artal Sanz, M., Bucko, M., Farhoud, M.H., Feenstra, M., Hakkaart, G.A., Zeviani, M., and Grivell, L.A. (2001). Shylp occurs in a high molecular weight complex and is required for efficient assembly of cytochrome c oxidase in yeast. FEBS Lett 498, 46-51.

Nijtmans, L.G., Taanman, J.W., Muijsers, A.O., Speijer, D., and Van den Bogert, C. (1998). Assembly of cytochrome-c oxidase in cultured human cells. Eur J Biochem 254, 389-394.

Nowaczyk, M.M., Hebeler, R., Schlodder, E., Meyer, H.E., Warscheid, B., and Rogner, M. (2006). Psb27, a cyanobacterial lipoprotein, is involved in the repair cycle of photosystem II. Plant Cell 18, 3121-3131.

Okamoto, K., and Shaw, J.M. (2005). Mitochondrial morphology and dynamics in yeast and multicellular eukaryotes. Annu Rev Genet 39, 503-536.

Oswald, C., Krause-Buchholz, U., and Rödel, G. (2009). Knockdown of human COX17 affects assembly and supramolecular organization of cytochrome c oxidase. J Mol Biol 389, 470-479.

Ott, M., and Herrmann, J.M. (2010). Co-translational membrane insertion of mitochondrially encoded proteins. Biochim Biophys Acta 1803, 767-775.

Palade, G.E. (1964). The Organization of Living Matter. Proc Natl Acad Sci U S A 52, 613-634. 
Palmieri, F., and Pierri, C.L. (2010). Mitochondrial metabolite transport. Essays Biochem 47, 37-52.

Palmisano, A., Zara, V., Honlinger, A., Vozza, A., Dekker, P.J., Pfanner, N., and Palmieri, F. (1998). Targeting and assembly of the oxoglutarate carrier: general principles for biogenesis of carrier proteins of the mitochondrial inner membrane. Biochem J 333 ( Pt 1), 151-158.

Pebay-Peyroula, E., Dahout-Gonzalez, C., Kahn, R., Trezeguet, V., Lauquin, G.J., and Brandolin, G. (2003). Structure of mitochondrial ADP/ATP carrier in complex with carboxyatractyloside. Nature 426, 39-44.

Pequignot, M.O., Dey, R., Zeviani, M., Tiranti, V., Godinot, C., Poyau, A., Sue, C., Di Mauro, S., Abitbol, M., and Marsac, C. (2001). Mutations in the SURF1 gene associated with Leigh syndrome and cytochrome C oxidase deficiency. Hum Mutat 17, 374-381.

Perez-Martinez, X., Broadley, S.A., and Fox, T.D. (2003). Mss51p promotes mitochondrial Cox1p synthesis and interacts with newly synthesized Cox1p. EMBO J 22, 5951-5961.

Petrakis, N., Alcock, F., and Tokatlidis, K. (2009). Mitochondrial ATP-independent chaperones. IUBMB Life $61,909-914$.

Pfanner, N., Hoeben, P., Tropschug, M., and Neupert, W. (1987). The carboxyl-terminal two-thirds of the ADP/ATP carrier polypeptide contains sufficient information to direct translocation into mitochondria. J Biol Chem 262, 14851-14854.

Pfanner, N., and Neupert, W. (1987). Distinct steps in the import of ADP/ATP carrier into mitochondria. J Biol Chem 262, 7528-7536.

Piekutowska-Abramczuk, D., Magner, M., Popowska, E., Pronicki, M., Karczmarewicz, E., Sykut-Cegielska, J., Kmiec, T., Jurkiewicz, E., Szymanska-Debinska, T., Bielecka, L., et al. (2009). SURF1 missense mutations promote a mild Leigh phenotype. Clin Genet 76, 195-204.

Pierrel, F., Bestwick, M.L., Cobine, P.A., Khalimonchuk, O., Cricco, J.A., and Winge, D.R. (2007). Coa1 links the Mss51 post-translational function to Cox1 cofactor insertion in cytochrome c oxidase assembly. EMBO J $26,4335-4346$.

Pithukpakorn, M. (2005). Disorders of pyruvate metabolism and the tricarboxylic acid cycle. Mol Genet Metab $85,243-246$.

Ponamarev, M.V., Longley, M.J., Nguyen, D., Kunkel, T.A., and Copeland, W.C. (2002). Active site mutation in DNA polymerase gamma associated with progressive external ophthalmoplegia causes error-prone DNA synthesis. J Biol Chem 277, 15225-15228.

Poyau, A., Buchet, K., Bouzidi, M.F., Zabot, M.T., Echenne, B., Yao, J., Shoubridge, E.A., and Godinot, C. (2000). Missense mutations in SURF1 associated with deficient cytochrome c oxidase assembly in Leigh syndrome patients. Hum Genet 106, 194-205.

Rahman, S., Blok, R.B., Dahl, H.H., Danks, D.M., Kirby, D.M., Chow, C.W., Christodoulou, J., and Thorburn, D.R. (1996). Leigh syndrome: clinical features and biochemical and DNA abnormalities. Ann Neurol 39, $343-$ 351.

Rehling, P., Brandner, K., and Pfanner, N. (2004). Mitochondrial import and the twin-pore translocase. Nat Rev Mol Cell Biol 5, 519-530.

Rehling, P., Model, K., Brandner, K., Kovermann, P., Sickmann, A., Meyer, H.E., Kühlbrandt, W., Wagner, R., Truscott, K.N., and Pfanner, N. (2003). Protein insertion into the mitochondrial inner membrane by a twin-pore translocase. Science 299, 1747-1751. 
Rigoulet, M., Yoboue, E.D., and Devin, A. (2011). Mitochondrial ROS generation and its regulation: mechanisms involved in $\mathrm{H}(2) \mathrm{O}(2)$ signaling. Antioxid Redox Signal 14, 459-468.

Rinaldo, P., Matern, D., and Bennett, M.J. (2002). Fatty acid oxidation disorders. Annu Rev Physiol 64, 477502.

Roesch, K., Curran, S.P., Tranebjaerg, L., and Koehler, C.M. (2002). Human deafness dystonia syndrome is caused by a defect in assembly of the DDP1/TIMM8a-TIMM13 complex. Hum Mol Genet 11, 477-486.

Rojo, E.E., Stuart, R.A., and Neupert, W. (1995). Conservative sorting of F0-ATPase subunit 9: export from matrix requires delta $\mathrm{pH}$ across inner membrane and matrix ATP. EMBO J 14, 3445-3451.

Rokka, A., Antonenkov, V.D., Soininen, R., Immonen, H.L., Pirilä, P.L., Bergmann, U., Sormunen, R.T., Weckström, M., Benz, R., and Hiltunen, J.K. (2009). Pxmp2 is a channel-forming protein in Mammalian peroxisomal membrane. PLoS ONE 4, e5090.

Röttgers, K., Zufall, N., Guiard, B., and Voos, W. (2002). The ClpB homolog Hsp78 is required for the efficient degradation of proteins in the mitochondrial matrix. J Biol Chem 277, 45829-45837.

Rousset, S., Alves-Guerra, M.C., Mozo, J., Miroux, B., Cassard-Doulcier, A.M., Bouillaud, F., and Ricquier, D. (2004). The biology of mitochondrial uncoupling proteins. Diabetes 53 Suppl 1, S130-135.

Ryan, M.T., Müller, H., and Pfanner, N. (1999). Functional staging of ADP/ATP carrier translocation across the outer mitochondrial membrane. J Biol Chem 274, 20619-20627.

Saada, A., Shaag, A., Mandel, H., Nevo, Y., Eriksson, S., and Elpeleg, O. (2001). Mutant mitochondrial thymidine kinase in mitochondrial DNA depletion myopathy. Nat Genet 29, 342-344.

Sacconi, S., Salviati, L., Sue, C.M., Shanske, S., Davidson, M.M., Bonilla, E., Naini, A.B., De Vivo, D.C., and DiMauro, S. (2003). Mutation screening in patients with isolated cytochrome c oxidase deficiency. Pediatr Res $53,224-230$.

Samuel, D. (1996). Investigation of Ancient Egyptian Baking and Brewing Methods by Correlative Microscopy. Science 273, 488-490.

Sasarman, F., Brunel-Guitton, C., Antonicka, H., Wai, T., and Shoubridge, E.A. (2010). LRPPRC and SLIRP interact in a ribonucleoprotein complex that regulates posttranscriptional gene expression in mitochondria. Mol Biol Cell 21, 1315-1323.

Schaefer, A.M., Taylor, R.W., Turnbull, D.M., and Chinnery, P.F. (2004). The epidemiology of mitochondrial disorders--past, present and future. Biochim Biophys Acta 1659, 115-120.

Schägger, H., and Pfeiffer, K. (2000). Supercomplexes in the respiratory chains of yeast and mammalian mitochondria. EMBO J 19, 1777-1783.

Schägger, H., and von Jagow, G. (1991). Blue native electrophoresis for isolation of membrane protein complexes in enzymatically active form. Anal Biochem 199, 223-231.

Schulz, C., Lytovchenko, O., Melin, J., Chacinska, A., Guiard, B., Neumann, P., Ficner, R., Jahn, O., Schmidt, B., and Rehling, P. (2011). Tim50's presequence receptor domain is essential for signal driven transport across the TIM23-complex. J Cell Biol in press.

Seo, A.Y., Joseph, A.M., Dutta, D., Hwang, J.C., Aris, J.P., and Leeuwenburgh, C. (2010). New insights into the role of mitochondria in aging: mitochondrial dynamics and more. J Cell Sci 123, 2533-2542. 
Shiflett, A.M., and Johnson, P.J. (2010). Mitochondrion-related organelles in eukaryotic protists. Annu Rev Microbiol 64, 409-429.

Sicard, D., and Legras, J.L. (2011). Bread, beer and wine: yeast domestication in the Saccharomyces sensu stricto complex. C R Biol 334, 229-236.

Sickmann, A., Reinders, J., Wagner, Y., Joppich, C., Zahedi, R., Meyer, H.E., Schönfisch, B., Perschil, I., Chacinska, A., Guiard, B., et al. (2003). The proteome of Saccharomyces cerevisiae mitochondria. Proc Natl Acad Sci USA 100, 13207-13212.

Sikorski, R.S., and Hieter, P. (1989). A system of shuttle vectors and yeast host strains designed for efficient manipulation of DNA in Saccharomyces cerevisiae. Genetics 122, 19-27.

Sirrenberg, C., Endres, M., Folsch, H., Stuart, R.A., Neupert, W., and Brunner, M. (1998). Carrier protein import into mitochondria mediated by the intermembrane proteins Tim10/Mrs11 and Tim12/Mrs5. Nature 391, 912-915.

Smeitink, J.A., Elpeleg, O., Antonicka, H., Diepstra, H., Saada, A., Smits, P., Sasarman, F., Vriend, G., JacobHirsch, J., Shaag, A., et al. (2006). Distinct clinical phenotypes associated with a mutation in the mitochondrial translation elongation factor EFTs. Am J Hum Genet 79, 869-877.

Smith, D., Gray, J., Mitchell, L., Antholine, W.E., and Hosler, J.P. (2005). Assembly of cytochrome-c oxidase in the absence of assembly protein Surflp leads to loss of the active site heme. J Biol Chem 280, 17652-17656.

Spelbrink, J.N., Li, F.Y., Tiranti, V., Nikali, K., Yuan, Q.P., Tariq, M., Wanrooij, S., Garrido, N., Comi, G., Morandi, L., et al. (2001). Human mitochondrial DNA deletions associated with mutations in the gene encoding Twinkle, a phage T7 gene 4-like protein localized in mitochondria. Nat Genet 28, 223-231.

Spinazzola, A., Santer, R., Akman, O.H., Tsiakas, K., Schaefer, H., Ding, X., Karadimas, C.L., Shanske, S., Ganesh, J., Di Mauro, S., et al. (2008). Hepatocerebral form of mitochondrial DNA depletion syndrome: novel MPV17 mutations. Arch Neurol 65, 1108-1113.

Spinazzola, A., Viscomi, C., Fernandez-Vizarra, E., Carrara, F., D'Adamo, P., Calvo, S., Marsano, R.M., Donnini, C., Weiher, H., Strisciuglio, P., et al. (2006). MPV17 encodes an inner mitochondrial membrane protein and is mutated in infantile hepatic mitochondrial DNA depletion. Nat Genet 38, 570-575.

Spinazzola, A., and Zeviani, M. (2007). Disorders of nuclear-mitochondrial intergenomic communication. Biosci Rep 27, 39-51.

Stiburek, L., Vesela, K., Hansikova, H., Pecina, P., Tesarova, M., Cerna, L., Houstek, J., and Zeman, J. (2005). Tissue-specific cytochrome c oxidase assembly defects due to mutations in SCO2 and SURF1. Biochem J 392, 625-632.

Stock, D., Gibbons, C., Arechaga, I., Leslie, A.G., and Walker, J.E. (2000). The rotary mechanism of ATP synthase. Curr Opin Struct Biol 10, 672-679.

Stojanovski, D., Müller, J.M., Milenkovic, D., Guiard, B., Pfanner, N., and Chacinska, A. (2008). The MIA system for protein import into the mitochondrial intermembrane space. Biochim Biophys Acta 1783, 610-617.

Sun, F., Huo, X., Zhai, Y., Wang, A., Xu, J., Su, D., Bartlam, M., and Rao, Z. (2005). Crystal structure of mitochondrial respiratory membrane protein complex II. Cell 121, 1043-1057.

Svensson-Ek, M., Abramson, J., Larsson, G., Tornroth, S., Brzezinski, P., and Iwata, S. (2002). The X-ray crystal structures of wild-type and EQ(I-286) mutant cytochrome c oxidases from Rhodobacter sphaeroides. J Mol Biol 321, 329-339. 
Szklarczyk, R., and Huynen, M.A. (2010). Mosaic origin of the mitochondrial proteome. Proteomics 10, 40124024.

Sztul, E.S., Hendrick, J.P., Kraus, J.P., Wall, D., Kalousek, F., and Rosenberg, L.E. (1987). Import of rat ornithine transcarbamylase precursor into mitochondria: two-step processing of the leader peptide. J Cell Biol 105, 2631-2639.

Takakubo, F., Cartwright, P., Hoogenraad, N., Thorburn, D.R., Collins, F., Lithgow, T., and Dahl, H.H. (1995). An amino acid substitution in the pyruvate dehydrogenase E1 alpha gene, affecting mitochondrial import of the precursor protein. Am J Hum Genet 57, 772-780.

Tal, R., Winter, G., Ecker, N., Klionsky, D.J., and Abeliovich, H. (2007). Aup1p, a yeast mitochondrial protein phosphatase homolog, is required for efficient stationary phase mitophagy and cell survival. J Biol Chem 282, 5617-5624.

Tatuch, Y., Christodoulou, J., Feigenbaum, A., Clarke, J.T., Wherret, J., Smith, C., Rudd, N., Petrova-Benedict, R., and Robinson, B.H. (1992). Heteroplasmic mtDNA mutation (T----G) at 8993 can cause Leigh disease when the percentage of abnormal mtDNA is high. Am J Hum Genet 50, 852-858.

Tavares-Carreon, F., Camacho-Villasana, Y., Zamudio-Ochoa, A., Shingu-Vazquez, M., Torres-Larios, A., and Perez-Martinez, X. (2008). The pentatricopeptide repeats present in Pet309 are necessary for translation but not for stability of the mitochondrial COX1 mRNA in yeast. J Biol Chem 283, 1472-1479.

Taylor, S.W., Fahy, E., Zhang, B., Glenn, G.M., Warnock, D.E., Wiley, S., Murphy, A.N., Gaucher, S.P., Capaldi, R.A., Gibson, B.W., et al. (2003). Characterization of the human heart mitochondrial proteome. Nat Biotechnol 21, 281-286.

Teraoka, M., Yokoyama, Y., Ninomiya, S., Inoue, C., Yamashita, S., and Seino, Y. (1999). Two novel mutations of SURF1 in Leigh syndrome with cytochrome c oxidase deficiency. Hum Genet 105, 560-563.

Tiranti, V., Hoertnagel, K., Carrozzo, R., Galimberti, C., Munaro, M., Granatiero, M., Zelante, L., Gasparini, P., Marzella, R., Rocchi, M., et al. (1998). Mutations of SURF-1 in Leigh disease associated with cytochrome c oxidase deficiency. Am J Hum Genet 63, 1609-1621.

Towpik, J. (2005). Regulation of mitochondrial translation in yeast. Cell Mol Biol Lett 10, 571-594.

Trott, A., and Morano, K.A. (2004). SYM1 is the stress-induced Saccharomyces cerevisiae ortholog of the mammalian kidney disease gene Mpv17 and is required for ethanol metabolism and tolerance during heat shock. Eukaryotic Cell 3, 620-631.

Truscott, K.N., Kovermann, P., Geissler, A., Merlin, A., Meijer, M., Driessen, A.J., Rassow, J., Pfanner, N., and Wagner, R. (2001). A presequence- and voltage-sensitive channel of the mitochondrial preprotein translocase formed by Tim23. Nat Struct Biol 8, 1074-1082.

Truscott, K.N., Voos, W., Frazier, A.E., Lind, M., Li, Y., Geissler, A., Dudek, J., Müller, H., Sickmann, A., Meyer, H.E., et al. (2003). A J-protein is an essential subunit of the presequence translocase-associated protein import motor of mitochondria. J Cell Biol 163, 707-713.

Truscott, K.N., Wiedemann, N., Rehling, P., Müller, H., Meisinger, C., Pfanner, N., and Guiard, B. (2002). Mitochondrial import of the ADP/ATP carrier: the essential TIM complex of the intermembrane space is required for precursor release from the TOM complex. Mol Cell Biol 22, 7780-7789.

Tsukihara, T., Aoyama, H., Yamashita, E., Tomizaki, T., Yamaguchi, H., Shinzawa-Itoh, K., Nakashima, R., Yaono, R., and Yoshikawa, S. (1996). The whole structure of the 13-subunit oxidized cytochrome c oxidase at 2.8 A. Science $272,1136-1144$. 
Turrens, J.F. (1997). Superoxide production by the mitochondrial respiratory chain. Biosci Rep 17, 3-8.

Turrens, J.F. (2003). Mitochondrial formation of reactive oxygen species. J Physiol 552, 335-344.

Valente, L., Tiranti, V., Marsano, R.M., Malfatti, E., Fernandez-Vizarra, E., Donnini, C., Mereghetti, P., De Gioia, L., Burlina, A., Castellan, C., et al. (2007). Infantile encephalopathy and defective mitochondrial DNA translation in patients with mutations of mitochondrial elongation factors EFG1 and EFTu. Am J Hum Genet $80,44-58$.

van der Giezen, M., and Tovar, J. (2005). Degenerate mitochondria. EMBO Rep 6, 525-530.

van der Laan, M., Hutu, D.P., and Rehling, P. (2010). On the mechanism of preprotein import by the mitochondrial presequence translocase. Biochim Biophys Acta 1803, 732-739.

van der Laan, M., Meinecke, M., Dudek, J., Hutu, D.P., Lind, M., Perschil, I., Guiard, B., Wagner, R., Pfanner, N., and Rehling, P. (2007). Motor-free mitochondrial presequence translocase drives membrane integration of preproteins. Nat Cell Biol 9, 1152-1159.

Van Veldhoven, P.P., Just, W.W., and Mannaerts, G.P. (1987). Permeability of the peroxisomal membrane to cofactors of beta-oxidation. Evidence for the presence of a pore-forming protein. J Biol Chem 262, 4310-4318.

Velours, G., Boucheron, C., Manon, S., and Camougrand, N. (2002). Dual cell wall/mitochondria localization of the 'SUN' family proteins. FEMS Microbiol Lett 207, 165-172.

Vögtle, F.-N., Wortelkamp, S., Zahedi, R.P., Becker, D., Leidhold, C., Gevaert, K., Kellermann, J., Voos, W., Sickmann, A., Pfanner, N., et al. (2009). Global analysis of the mitochondrial N-proteome identifies a processing peptidase critical for protein stability. Cell 139, 428-439.

Vreken, P., Valianpour, F., Nijtmans, L.G., Grivell, L.A., Plecko, B., Wanders, R.J., and Barth, P.G. (2000). Defective remodeling of cardiolipin and phosphatidylglycerol in Barth syndrome. Biochem Biophys Res Commun 279, 378-382.

Wagner, G., Stettmaier, K., Bors, W., Sies, H., Wagner, E.M., Reuter, A., and Weiher, H. (2001). Enhanced gamma-glutamyl transpeptidase expression and superoxide production in Mpv17-/- glomerulosclerosis mice. Biol Chem 382, 1019-1025.

Wagner, K., Gebert, N., Guiard, B., Brandner, K., Truscott, K.N., Wiedemann, N., Pfanner, N., and Rehling, P. (2008). The assembly pathway of the mitochondrial carrier translocase involves four preprotein translocases. Mol Cell Biol 28, 4251-4260.

Wallace, D.C. (2007). Why do we still have a maternally inherited mitochondrial DNA? Insights from evolutionary medicine. Annu Rev Biochem 76, 781-821.

Wallace, D.C., Singh, G., Lott, M.T., Hodge, J.A., Schurr, T.G., Lezza, A.M., Elsas, L.J., 2nd, and Nikoskelainen, E.K. (1988). Mitochondrial DNA mutation associated with Leber's hereditary optic neuropathy. Science 242, 1427-1430.

Wang, P., and Dalbey, R.E. (2011). Inserting membrane proteins: the YidC/Oxa1/Alb3 machinery in bacteria, mitochondria, and chloroplasts. Biochim Biophys Acta 1808, 866-875.

Webb, C.T., Gorman, M.A., Lazarou, M., Ryan, M.T., and Gulbis, J.M. (2006). Crystal structure of the mitochondrial chaperone TIM9.10 reveals a six-bladed alpha-propeller. Mol Cell 21, 123-133.

Weiher, H., Noda, T., Gray, D.A., Sharpe, A.H., and Jaenisch, R. (1990). Transgenic mouse model of kidney disease: insertional inactivation of ubiquitously expressed gene leads to nephrotic syndrome. Cell 62, 425-434. 
Weraarpachai, W., Antonicka, H., Sasarman, F., Seeger, J., Schrank, B., Kolesar, J.E., Lochmüller, H., Chevrette, M., Kaufman, B.A., Horvath, R., et al. (2009). Mutation in TACO1, encoding a translational activator of COX I, results in cytochrome c oxidase deficiency and late-onset Leigh syndrome. Nat Genet 41, 833-837.

Westermann, B., and Neupert, W. (1997). Mdj2p, a novel DnaJ homolog in the mitochondrial inner membrane of the yeast Saccharomyces cerevisiae. J Mol Biol 272, 477-483.

Wiedemann, N., Pfanner, N., and Chacinska, A. (2006a). Chaperoning through the mitochondrial intermembrane space. Mol Cell 21, 145-148.

Wiedemann, N., Pfanner, N., and Rehling, P. (2006b). Import of precursor proteins into isolated yeast mitochondria. Methods Mol Biol 313, 373-383.

Wiedemann, N., Pfanner, N., and Ryan, M.T. (2001). The three modules of ADP/ATP carrier cooperate in receptor recruitment and translocation into mitochondria. EMBO J 20, 951-960.

Williams, S.L., Valnot, I., Rustin, P., and Taanman, J.W. (2004). Cytochrome c oxidase subassemblies in fibroblast cultures from patients carrying mutations in COX10, SCO1, or SURF1. J Biol Chem 279, 7462-7469.

Wittig, I., Braun, H.P., and Schägger, H. (2006). Blue native PAGE. Nat Protoc 1, 418-428.

Wittig, I., and Schägger, H. (2009). Supramolecular organization of ATP synthase and respiratory chain in mitochondrial membranes. Biochim Biophys Acta 1787, 672-680.

Wong, L.-J.C., Brunetti-Pierri, N., Zhang, Q., Yazigi, N., Bove, K.E., Dahms, B.B., Puchowicz, M.A., Gonzalez-Gomez, I., Schmitt, E.S., Truong, C.K., et al. (2007). Mutations in the MPV17 gene are responsible for rapidly progressive liver failure in infancy. Hepatology 46, 1218-1227.

Xia, D., Yu, C.A., Kim, H., Xia, J.Z., Kachurin, A.M., Zhang, L., Yu, L., and Deisenhofer, J. (1997). Crystal structure of the cytochrome bc1 complex from bovine heart mitochondria. Science 277, 60-66.

Xu, Y., Malhotra, A., Ren, M., and Schlame, M. (2006). The enzymatic function of tafazzin. J Biol Chem 281, 39217-39224.

Yaffe, M.P., and Schatz, G. (1984). Two nuclear mutations that block mitochondrial protein import in yeast. Proc Natl Acad Sci USA 81, 4819-4823.

Yamamoto, H., Esaki, M., Kanamori, T., Tamura, Y., Nishikawa, S., and Endo, T. (2002). Tim50 is a subunit of the TIM23 complex that links protein translocation across the outer and inner mitochondrial membranes. Cell $111,519-528$.

Yang, Y.L., Sun, F., Zhang, Y., Qian, N., Yuan, Y., Wang, Z.X., Qi, Y., Xiao, J.X., Wang, X.Y., Qi, Z.Y., et al. (2006). Clinical and laboratory survey of 65 Chinese patients with Leigh syndrome. Chin Med J (Engl) 119, 373-377.

Yu-Wai-Man, P., Griffiths, P.G., Hudson, G., and Chinnery, P.F. (2009). Inherited mitochondrial optic neuropathies. J Med Genet 46, 145-158.

Yuksel, A., Seven, M., Cetincelik, U., Yesil, G., and Koksal, V. (2006). Facial dysmorphism in Leigh syndrome with SURF-1 mutation and COX deficiency. Pediatr Neurol 34, 486-489.

Zeviani, M., and Carelli, V. (2007). Mitochondrial disorders. Curr Opin Neurol 20, 564-571.

Zhang, Z., and Dietrich, F.S. (2005). Mapping of transcription start sites in Saccharomyces cerevisiae using 5' SAGE. Nucleic Acids Res 33, 2838-2851. 
Zhu, Z., Yao, J., Johns, T., Fu, K., De Bie, I., Macmillan, C., Cuthbert, A.P., Newbold, R.F., Wang, J., Chevrette, M., et al. (1998). SURF1, encoding a factor involved in the biogenesis of cytochrome c oxidase, is mutated in Leigh syndrome. Nat Genet 20,337-343.

Zick, M., Rabl, R., and Reichert, A.S. (2009). Cristae formation-linking ultrastructure and function of mitochondria. Biochim Biophys Acta 1793, 5-19.

Zwacka, R.M., Reuter, A., Pfaff, E., Moll, J., Gorgas, K., Karasawa, M., and Weiher, H. (1994). The glomerulosclerosis gene Mpv17 encodes a peroxisomal protein producing reactive oxygen species. EMBO J 13, 5129-5134. 


\section{Acknowledgements}

Zuallererst möchte ich mich bei Prof. Dr. Peter Rehling für die exzellente Betreuung meiner Arbeit, seine ständige Diskussionsbereitschaft und die vielen Ideen bedanken, ohne die diese Arbeit nicht möglich gewesen wäre.

Für die Betreuung dieser Arbeit im Rahmen der Thesis-Committee Sitzungen bedanke ich mich bei Prof. Dr. Michael Thumm und Prof. Dr. Mikael Simons, dem mein Dank auch für die Anfertigung des Zweitgutachtens gilt.

Ich bedanke mich bei allen ehemaligen und aktuellen Mitarbeitern der Arbeitsgruppe Rehling für das angenehme Arbeitsklima und allerlei Hilfen bei den täglichen Herausforderungen im Labor. Ganz besonderer Dank gilt dabei Dr. David U. Mick, Dr. Markus Deckers, Dr. Jan Dudek, Dr. Sven Dennerlein, Christian Schulz und Bettina Bareth. Außerdem bedanke ich mich für die Unterstützung von Martina Balleininger, Mirjam Wissel, Petra Engelmann und den Mitarbeitern der wissenschaftlichen Werkstätten.

Für die Zusammenarbeit bei den elektrophysiologischen Untersuchungen möchte ich mich bei Prof. Dr. Richard Wagner, Dr. Michael Meinecke und Dr. Vivien Krüger bedanken.

Außerdem danke ich Dr. Martin van der Laan und Inge Perschill für die methodische Einführung ins Labor.

Ganz besonderer Dank gilt meiner Familie für die Unterstützung und Ermutigung während meiner Arbeit hier in Göttingen. Von ganzem Herzen möchte ich auch Evi danken, die immer für mich da war. 


\section{Curriculum Vitae}

\section{Personal information}

Name

Born

\section{Education}

Since $02 / 2008$

$02 / 2008-04 / 2008$

$10 / 2002-12 / 2007$

Technische Universität Dresden, Germany

Diploma in Biology (Genetics, Microbiology, Biochemistry)

Thesis: Mitochondriale Biogenese in Saccharomyces cerevisiae:

Molekularbiologische Charakterisierung der Proteinkinase Pho85p

Supervisor: Prof. Dr. Gerhard Rödel

10/2005 - 03/2006 Guest student at the University of York, UK 